\title{
Interplay of martensitic phase transformation and plastic slip in polycrystals
}

Thesis by

Andrew Walter Richards

In Partial Fulfillment of the Requirements

for the Degree of

Doctor of Philosophy

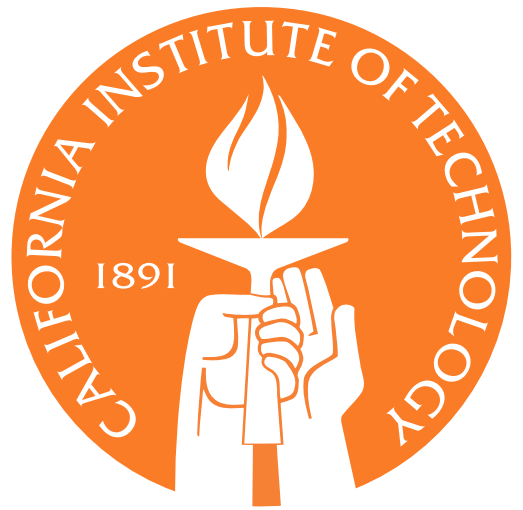

California Institute of Technology

Pasadena, California

2013

(Defended May 1, 2013) 
(c) 2013

Andrew Walter Richards

All Rights Reserved 
To Rachel. 


\section{Acknowledgments}

This material is based upon work supported by the Department of Energy National Nuclear Security Administration under Award Number DE-FC52-08NA28613.

With much love, I thank my parents, Larry and Linda Richards, and my brothers, Keith and Frank, for their support during my protracted academic pursuits.

I am deeply indebted to my advisor, Prof. Kaushik Bhattacharya, for his clear guidance (and abundant patience) throughout my studies at Caltech. His influence on my academics actually predates my time at Caltech, when he visited my alma mater (Michigan Technological University) in March of 2006. I had recently suspended my Master's studies on electrostrictive polymers to begin working full-time as a research engineer on an unrelated DARPA project, and his recommendation of W.F. Brown, Jr.'s monograph on magnetoelasticity proved invaluable when I eventually resumed my studies. It was this interaction (and encouragement from my boss at the time, Prof. Ghatu Subhash) that precipitated my move to Caltech to pursue a Ph.D. I fear I will never be able to adequately express how grateful I am for the insights he has given me.

I would like to thank Dr. Ricardo Lebensohn for flying out to serve on my thesis 
committee, and for mentoring me during my first internship at Los Alamos National Laboratory (LANL) during the summer of 2009. The work we started that summer (and the subsequent journal article we co-authored with Prof. Bhatacharya) went on to form the backbone of this thesis.

I shall be eternally grateful to Prof. Guruswami Ravichandran for his insights on all matters practical and philosophical (as well as for serving on my thesis committee). I also thank Prof. Michael Ortiz for serving as my thesis committee chair, and for providing guidance and support through Caltech's Predictive Science Academic Alliance (PSAAP) program.

The large scale simulations presented in this work would have been impossible to complete without the help of Bo Li. I am also indebted to Daniel Hurtado for helping me learn the ins and outs of programming in $\mathrm{C}++$ and Eureka.

I wish to thank Dingyi Sun for providing me with a script which will be used for future investigations of intragranular inclusions, and Laurence Bodelot, whose ongoing experimental collaboration into the initiation of plasticity in polycrystals I am eager resume. I would also like to thank Dr. Dean Preston who mentored me during my second internship at LANL during summer of 2011, and I will be forever grateful to Dr. Turab Lookman who took me into his home when the town of Los Alamos was evacuated during the Las Conchas Fire. I further thank my Master's thesis advisor at Michigan Tech, Prof. Gregory Odegard, and the best supervisor I could ever hope for, James B. Lewis, for encouraging me to pursue my Ph.D. 
I would like to thank all of the members of the Bhattacharya \& Ravichandran Groups, past and present, whom I have had the privilege of working with, but especially Bharat Penmecha, Phanish Suryanarayana, Aaron Stebner, Md. Zubaer Hossain, Cindy Wang, and Jacob Notbohm, for their patience when I needed to talk myself through a problem. I would also like to thank Leslie Rico for her help in coordinating meetings (and generally keeping tabs on my well-being), and Susan Powell, for coordinating my travels to LANL (and all things PSAAP).

A very heartfelt thank-you goes to my friends Ian Jacobi, Jonathan Mihaly, Nicholas Boechler, Mumu Xu, Justin Brown, Esperanza Linares, Christine Winiarz Schmidt, Paula Popescu (\& the TND group), and Daniel Jones for all their help in keeping me sane both inside and outside the classroom.

And lastly, I would like to thank Dr. Bill \& Dolores Bing, Sarah Milkovich, Catherine Wehrey, and Jeffrey Thompson for allowing me to continue making music (and desserts) while at Caltech. From performing together at Carnegie Hall and the Great Wall of China, to sharing countless meals together, the opportunities and relationships afforded me by the Caltech music programs will be forever cherished. 


\section{Abstract}

Inspired by key experimental and analytical results regarding Shape Memory Alloys (SMAs), we propose a modelling framework to explore the interplay between martensitic phase transformations and plastic slip in polycrystalline materials, with an eye towards computational efficiency. The resulting framework uses a convexified potential for the internal energy density to capture the stored energy associated with transformation at the meso-scale, and introduces kinetic potentials to govern the evolution of transformation and plastic slip. The framework is novel in the way it treats plasticity on par with transformation.

We implement the framework in the setting of anti-plane shear, using a staggered implicit/explict update: we first use a Fast-Fourier Transform (FFT) solver based on an Augmented Lagrangian formulation to implicitly solve for the full-field displacements of a simulated polycrystal, then explicitly update the volume fraction of martensite and plastic slip using their respective stick-slip type kinetic laws. We observe that, even in this simple setting with an idealized material comprising four martensitic variants and four slip systems, the model recovers a rich variety of SMA type behaviors. We use this model to gain insight into the isothermal behavior of stress-stabilized martensite, looking at the 
effects of the relative plastic yield strength, the memory of deformation history under non-proportional loading, and several others.

We extend the framework to the generalized 3-D setting, for which the convexified potential is a lower bound on the actual internal energy, and show that the fully implicit discrete time formulation of the framework is governed by a variational principle for mechanical equilibrium. We further propose an extension of the method to finite deformations via an exponential mapping. We implement the generalized framework using an existing Optimal Transport Mesh-free (OTM) solver. We then model the $\alpha-\gamma$ and $\alpha-\varepsilon$ transformations in pure iron, with an initial attempt in the latter to account for twinning in the parent phase. We demonstrate the scalability of the framework to large scale computing by simulating Taylor impact experiments, observing nearly linear (ideal) speed-up through 256 MPI tasks. Finally, we present preliminary results of a simulated Split-Hopkinson Pressure Bar (SHPB) experiment using the $\alpha-\varepsilon$ model. 


\section{Contents}

Acknowledgments $\quad$ iv

$\begin{array}{ll}\text { Abstract } & \text { vii }\end{array}$

1 Introduction 1

1.1 Deformation mechanisms and effects . . . . . . . . . . . . . 4

1.1.1 Martensitic phase transformations . . . . . . . . . . . . 4

1.1 .2 Plastic slip . . . . . . . . . . . . . . . . 4

1.1.3 Shape memory and superelasticity . . . . . . . . . . . . 6

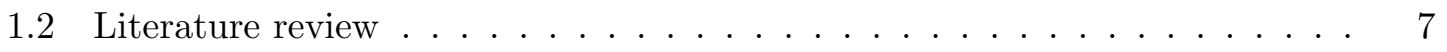

1.3 Chapter summary . . . . . . . . . . . . . . . . . . . . 12

2 Background $\quad 16$

2.1 Continuum kinematics . . . . . . . . . . . . . . . . . 16

2.2 Crystallography of martensite . . . . . . . . . . . . . . . . . 19

2.3 Crystal plasticity . . . . . . . . . . . . . . . . . . 22 
3 Antiplane Shear Model $\quad 25$

3.1 Kinematics . . . . . . . . . . . . . . . . . 25

3.2 Balance laws . . . . . . . . . . . . . . . . . . . . . . 28

3.3 Constitutive framework . . . . . . . . . . . . . . . . . 29

3.4 Isothermal setting and constitutive law . . . . . . . . . . . . . . 31

3.5 Staggered update and FFT Solver . . . . . . . . . . . . . . . 33

3.6 Enforcement of constraints . . . . . . . . . . . . . . . . . 38

4 Anti-plane Shear Results $\quad 39$

4.1 Standard specimen . . . . . . . . . . . . . . . . . . . 39

4.2 Uniaxial loading . . . . . . . . . . . . . . . . . . . 43

4.2 .1 Superelasticity . . . . . . . . . . . . . . . . . . 43

4.2.2 Interplay between transformation and plasticity . . . . . . . . . 48

4.2 .3 The effect of flow stress . . . . . . . . . . . . . . 55

4.2 .4 Specimen variation . . . . . . . . . . . . . . . 57

4.2.4.1 Textured specimens . . . . . . . . . . . . . 58

4.2 .5 Loading rate . . . . . . . . . . . . . . . . . . 58

4.3 Multiaxial and nonproportional straining f . . . . . . . . . . . 61

5 Generalized Framework $\quad 67$

5.1 Linearized kinematics in $3-\mathrm{D} \ldots \ldots \ldots \ldots \ldots$

5.2 Governing equations . . . . . . . . . . . . . . . 68 
5.3 Implicit time discretization and variational principle $\ldots \ldots \ldots \ldots$

5.4 Finite kinematics . . . . . . . . . . . . . . . . . . 75

6 Iron Model: Implementation \& Results $\quad 80$

$6.1 \alpha-\gamma$ transformation model . . . . . . . . . . . . . . . . . 81

$6.1 .1 \gamma$-Transformation systems . . . . . . . . . . . . . 82

$6.1 .2 \quad$ Plastic slip systems . . . . . . . . . . . . . . . 83

6.1 .3 Elastic energy density . . . . . . . . . . . . . . . . 83

6.1.4 Transformation stored energy . . . . . . . . . . . . . 85

6.1 .5 Pastic work-hardening . . . . . . . . . . . . 86

6.1 .6 Kinetic laws . . . . . . . . . . . . . . . . . . . . . 87

6.1.7 Temperature update . . . . . . . . . . . . . . . . 88

6.1.8 Constraint enforcement . . . . . . . . . . . . . . . 88

6.1.9 Active slip and transformation systems . . . . . . . . . . . 89

6.2 Taylor impact . . . . . . . . . . . . . . . . . . . . . 90

6.2 .1 Simulation results $\ldots \ldots \ldots \ldots$. . . . . . . . . . . . 91

6.2 .2 Scalability study . . . . . . . . . . . . . . . . . 95

$6.3 \varepsilon$-iron and $\alpha$-twinning $\ldots \ldots \ldots \ldots \ldots \ldots$

6.3 .1 Constitutive law . . . . . . . . . . . . . . . . . 101

6.4 Taylor bar: $\alpha-\varepsilon \ldots \ldots \ldots \ldots \ldots \ldots$. . . . . . . . . . . . . . . . . . . . . . . .

6.5 Split-Hopkinson Pressure Bar . . . . . . . . . . . . . . . 105 
xii

7 Conclusions \& Future Work

122

Bibliography

130 


\section{List of Figures}

1.1 Typical stress strain response of a superelastc material. The superelastic response is shown in the solid curve. If the material is strained beyond a certain limit, one has incomplete memory as shown in the dashed curve . . .

1.2 Response of pure iron at various strain rates. (From [76]. Reprinted with

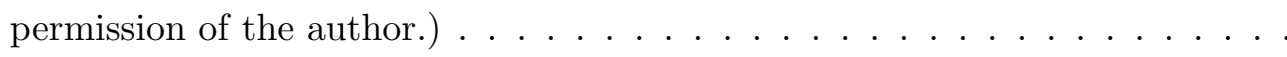

1.3 (a) Martensitic phase transformations. (b) Evolution of a non-convex energy potential as function of temperature (adapted from [27].) (c) Resulting stressstrain response of a single crystal associated with stress-stabilized martensite (from red or green curves in $(b)) \ldots \ldots \ldots \ldots$ 
xiv

1.4 Schematic of the shape memory effect. At high termperature, the material is in the highly symmetric austenite phase (red). Upon cooling, mixtures of martensite (blue and cyan) form such that shape of the specimen, on average, remains undeformed. During loading cycles, the martensite variants reapportion themselves, leading to residual deformations after unloading. Upon reheating, all variants of martensite revert back to the same parent phase, recovering the apparent permanent deformations. . . . . . . . . . . . 6

1.5 Schematic of superleasticity. At high temperatures, loading of the austenite phase can generate stress-stabilized martensite, which reduces the energy required to accomodate an applied deformation. The material returns to the austensite during unloading. . . . . . . . . . . . . . .

2.1 Variants of martensite in a cubic to tetragonal transformation. (From [11]. Reprinted with permission from author.) . . . . . . . . . . . 20

$2.2 \quad$ Stress-free intermediate configuration associated with plasticity. . . . . . . . 22 
4.1 (a) The standard polycrystal with 512 grains, and a zoomed in region where we display strain fields in subsequent figures. (b) The transformation surface of the standard material, and an arbitrary plastic yield surface. The same transformation surface was used for all of the results presented herein, but $\tau_{c}$ was varied from $141 \mathrm{MPa}$ to $\infty$. There are three transformation surfaces, the outer (respectively middle and inner) corresponding to the forward (respectively thermodynamic and reverse) transformation. . . . . . . . . .

4.2 Superelasticity with no plasticity. (a) The overall stress-strain relation. (b) The transformation strain field at the macroscopic initiation of transformation, at the beginning of reorientation and at the macroscopic saturation of the transformation. . . . . . . . . . . . . . . . . . 44

4.3 The interplay between transformation and plasticity under the cycling load protocol for a critical resolved stress of $424 \mathrm{MPa}$. (a) The stress-strain response of a protocol where the specimen is loaded in strain-control to $4 \%$ macroscopic strain, unloaded in strain-control to zero stress and then reloaded in strain-control cycles between zero stress and the value of the stress at the end of the first strain-control cycle. (b) The strain fields corresponding to six points. In each panel, the left column displays the transformation strain while the right column displays the plastic strain. . . . . . . . . . . . . 47 
xvi

4.4 Statistical features of transformation and plasticity for the case shown in Figure 4.3. (a) Heat map showing the distribution of transformation volume fraction and plastic activity as a function of grain orientation. A white pixel represents no region at that orientation and volume fraction while blue to red pixels represent increasing regions with that orientation and volume fraction. (b) Cumulative histogram of transformation and plastic activity vs. the nominal driving force. . . . . . . . . . . . . . . . . . . . 50

4.5 The interplay between transformation and plasticity under the cycling load protocol for a critical resolved stress of $283 \mathrm{MPa}$. (a) The stress-strain response of a loading protocol described in Figure 4.5. (b) The strain fields corresponding to six points. In each panel, the left column displays the transformation strain while the right column displays the plastic strain. . . . . . . 53

4.6 The interplay between transformation and plasticity under a increasing strain protocol for a critical resolved stress of $424 \mathrm{MPa}$. The specimen is loaded in strain control to $1 \%$, unloaded to zero stress and reloaded in strain control until an overall strain of $2 \%, 5 \%$ and $10 \%$. The dashed line shows the response of a virgin material strained to $10 \% \ldots \ldots \ldots \ldots$. . . . . . . . 54

4.7 Decomposition of strain components for various yield strengths. . . . . . . 55

4.8 The evolution of the residual strain with cycling for various values of the critical resolved stress. . . . . . . . . . . . . . . . . . 56

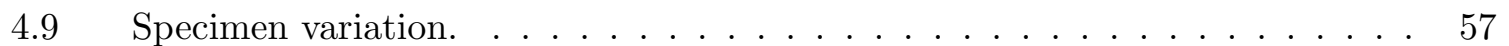


xvii

4.10 Effects of texture. (a) Gradient microstructure where the orientation and spatial location are highly correlated. (b) Comparison of macroscopic response of specimens with gradient microstructures (black and gray lines) to random microstructures. . . . . . . . . . . . . . . . . . 59

4.11 Uniaxial loading; reference loading rate (black) is $50 \mu \epsilon / \mathrm{s} . \ldots \ldots$. . . . . . 60

4.12 Decomposition of strain components for various loading rates. . . . . . . . . 60

4.13 Five multiaxial straining protocols. . . . . . . . . . . . . . . . . 61

4.14 The evolution of the stress when the standard specimen with critical resolved stress of $424 \mathrm{MPa}$ is subjected to multiaxial straining according to protocols XYyx, YXxy and Pp till a total strain of 1\%,2\%, 5\% and 10\%. The red colored curves show protocol XYyx, the green curves show the protocol YXxy and the blue diagonal lines show the protocol Pp. . . . . . . . . . . . 63

4.15 The evolution of the stress when the standard specimen with critical resolved stress of $300 \mathrm{MPa}$ is subjected to multiaxial straining according to protocols YXxy and XYxy till a total strain of 1\%,2\%,5\% and 10\%. The red colored curves show protocol XYxy, the green curves show the protocol YXxy and the blue curves show the protocol Pxy. . . . . . . . . . . . . . . 65

6.1 Idealized phase diagram for pure iron. (Adapted from [30].) . . . . . . . . . 81 
xviii

6.2 Taylor impact simulation of an iron bar for $\alpha-\gamma$ transformation at $227 \mathrm{~m} / \mathrm{s}$ (from scalability study). (Step 2.) The left field shows the von Mises stress; the center field shows the accumlated slip, summed over all systems; the right field shows the total martensite volume fraction, summed over all variants. We see the elastic precursor propagating upwards. . . . . . . . . . . . . . 91

6.3 Taylor impact simulation of an iron bar for $\alpha-\gamma$ transformation at $227 \mathrm{~m} / \mathrm{s}$ (from scalability study). (Step 3.) Initiation of plasticity and transformation is seen at the base of the specimen. . . . . . . . . . . . . . . 92

6.4 Taylor impact simulation of an iron bar for $\alpha-\gamma$ transformation at $227 \mathrm{~m} / \mathrm{s}$ (from scalability study). (Step 9.) We see the onset of the inelastic wave, propagating upwards. . . . . . . . . . . . . . . . . . . . 92

6.5 Taylor impact simulation of an iron bar for $\alpha-\gamma$ transformation at $227 \mathrm{~m} / \mathrm{s}$ (from scalability study). (Step 11.) The inelastic wave continues to propagate. We note that isolated transformation is visible ahead of the plastic wave, but that extensive transformation lags the plastic wavefront. . . . . . . . . . 93

6.6 Taylor impact simulation of an iron bar for $\alpha-\gamma$ transformation at $227 \mathrm{~m} / \mathrm{s}$ (from scalability study). (Step 16.) We see the elastic wave reaching the end

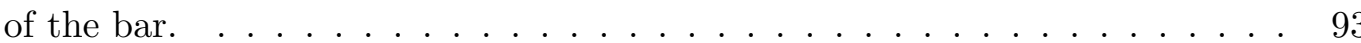

6.7 Taylor impact simulation of an iron bar $\alpha-\gamma$ transformation at $227 \mathrm{~m} / \mathrm{s}$ (from scalability study). (Step 21.) The elastic wave has reflected as a tensile wave, propagating downwards. . . . . . . . . . . . . . . . . 94 
xix

6.8 Taylor impact simulation of an iron bar $\alpha-\gamma$ transformation at $227 \mathrm{~m} / \mathrm{s}$ (from scalability study). We see the reflected wave tensile wave meeting the plastic wave front. . . . . . . . . . . . . . . . . . . . 94

6.9 Scalability study of the generalized framework for a Taylor anvil impact simulation of an iron bar. [Figure courtesy of Dr. B. Li.] . . . . . . . . . . . 96

6.10 Taylor anvil impact simulation of an iron bar for $\alpha-\varepsilon$ transformation at $227 \mathrm{~m} / \mathrm{s}$. (Step 2.) The left field shows the von Mises stress; the center field shows the accumlated slip, summed over all systems; the right field shows the total martensite volume fraction, summed over all variants. We see the elastic precursor travelling upwards, and initiation of plasticity at the base of the specimen. . . . . . . . . . . . . . . . . . 103

6.11 Taylor impact simulation of an iron bar for $\alpha-\varepsilon$ transformation at $227 \mathrm{~m} / \mathrm{s}$. (Step 3.) We see the onset of a plastic wave, propagating upwards.) . . . . . 104

6.12 Taylor impact simulation of an iron bar for $\alpha-\varepsilon$ transformation at $227 \mathrm{~m} / \mathrm{s}$. (Step 7.) We see the plastic wave continue, and note that no transformation or twinning is observed. . . . . . . . . . . . . . . . . 104

6.13 Simulated Taylor bar for $\alpha-\varepsilon$ transformation in iron at (a) $1000 \mathrm{~m} / \mathrm{s}$, and (b) $500 \mathrm{~m} / \mathrm{s}$, showing "melt" before transformation. . . . . . . . . . . . . 106

6.14 Schematic for Split-Hopkinson Pressure Bar. . . . . . . . . . . . . . . 107 
6.15 SHPB simulation of an iron specimen for $\alpha-\varepsilon$ transformation at $50 \mathrm{~m} / \mathrm{s}$. (Step 15.) The fields depict a cut-away view along an axisymmetric cylinder. The top left field shows the axis stress; the top right field shows the accumlated slip, summed over all systems; the bottom left field shows the total martensite volume fraction, summed over all variants; the bottom right field shows the total austenite twin volume fraction, summed over all variants. The incident and output bars are depicted as solid, and colormapped by the axial stress in each of the panels. The specimen is depicted by spherical glyphs at the material points. The incoming compression pulse travels from lower-left to upper right in the figure. . . . . . . . . . . . . . . . . 110

6.16 SHPB simulation of an iron specimen for $\alpha-\varepsilon$ transformation at $50 \mathrm{~m} / \mathrm{s}$. (Step 20.) The pulse reaches the specimen, which deforms elastically. . . . . . . . . 111

6.17 SHPB simulation of an iron specimen for $\alpha-\varepsilon$ transformation at $50 \mathrm{~m} / \mathrm{s}$. (Step 25.) The pressure pulse continues, and stresses can be seen localizing along the top of the specimen as radial deformations become significant. The specimen response is still elastic, however. . . . . . . . . . . . . . . . . . 111

6.18 SHPB simulation of an iron specimen for $\alpha-\varepsilon$ transformation at $50 \mathrm{~m} / \mathrm{s}$. (Step 30.) Plasticity is seen to initiate along interior planes in the specimen. . . . . 112

6.19 SHPB simulation of an iron specimen for $\alpha-\varepsilon$ transformation at $50 \mathrm{~m} / \mathrm{s}$. (Step

35.) Bands of plasticity begin to develop. . . . . . . . . . . . . . . 112 
xxi

6.20 SHPB simulation of an iron specimen for $\alpha-\varepsilon$ transformation at $50 \mathrm{~m} / \mathrm{s}$. (Step

40.) Bands of plasticity continue to develop. . . . . . . . . . . . . . . 113

6.21 SHPB simulation of an iron specimen for $\alpha-\varepsilon$ transformation at $50 \mathrm{~m} / \mathrm{s}$. (Step

45.) Bands of plasticity continue to develop. . . . . . . . . . . . . . . . . . 113

6.22 SHPB simulation of an iron specimen for $\alpha-\varepsilon$ transformation at $50 \mathrm{~m} / \mathrm{s}$. (Step 50.) An elastic tensile pulse reflected from the free end of the output bar can be seen in the upper right corner, travelling back down bar from top-right to bottom left. . . . . . . . . . . . . . . . . . . . . 114

6.23 SHPB simulation of an iron specimen for $\alpha-\varepsilon$ transformation at $100 \mathrm{~m} / \mathrm{s}$. (Step 15.) The fields depict a cut-away view along an axisymmetric cylinder. The top left field shows the axis stress; the top right field shows the accumlated slip, summed over all systems; the bottom left field shows the total martensite volume fraction, summed over all variants; the bottom right field shows the total austenite twin volume fraction, summed over all variants. The incident and output bars are depicted as solid, and colormapped by the axial stress in each of the panels. The specimen is depicted by spherical glyphs at the material points. The incoming compression pulse travels from lower-left to upper right in the figure. . . . . . . . . . . . . . . . . . 115

6.24 SHPB simulation of an iron specimen for $\alpha-\varepsilon$ transformation at $100 \mathrm{~m} / \mathrm{s}$. (Step 20.) The pulse reaches the specimen, which deforms elastically. . . . . 116 
xxii

6.25 SHPB simulation of an iron specimen for $\alpha-\varepsilon$ transformation at $100 \mathrm{~m} / \mathrm{s}$. (Step 25.) The pressure pulse continues, and stresses can be seen localizing along the top of the specimen as radial deformations become significant. Stress lobes near the specimen interface are evident in both the specimen and the bar. The specimen response is still mostly elastic, however. . . . . . . . 116

6.26 SHPB simulation of an iron specimen for $\alpha-\varepsilon$ transformation at $100 \mathrm{~m} / \mathrm{s}$. (Step 30.) A "wedge" of plasticity is seen to initiate along interior planes in the specimen, but still no transformation. . . . . . . . . . . . . . 117

6.27 SHPB simulation of an iron specimen for $\alpha-\varepsilon$ transformation at $100 \mathrm{~m} / \mathrm{s}$. (Step 35.) Bands of plasticity continue to develop. . . . . . . . . . . . . . 117

6.28 SHPB simulation of an iron specimen for $\alpha-\varepsilon$ transformation at $100 \mathrm{~m} / \mathrm{s}$. (Step 40.) Bands of plasticity continue to develop. . . . . . . . . . . . . . 118

6.29 SHPB simulation of an iron specimen for $\alpha-\varepsilon$ transformation at $100 \mathrm{~m} / \mathrm{s}$. (Step 45.) An isolated spot of transformation is seen, just below the surface of the specimen, near the edge of the incident bar. . . . . . . . . . . 118

6.30 SHPB simulation of an iron specimen for $\alpha-\varepsilon$ transformation at $100 \mathrm{~m} / \mathrm{s}$. (Step 50.) An elastic tensile pulse reflected from the free end of the output bar can be seen in the upper right corner, travelling back down bar from topright to bottom left. More transformation is seen to develop, mostly along the edges of the interfaces with the incident and output bars. . . . . . . . . 119 
xxiii

6.31 SHPB simulation of an iron specimen for $\alpha-\varepsilon$ transformation at $1000 \mathrm{~m} / \mathrm{s}$. (Step 15.) The fields depict a cut-away view along an axisymmetric cylinder. The top left field shows the axis stress; the top right field shows the accumlated slip, summed over all systems; the bottom left field shows the total martensite volume fraction, summed over all variants; the bottom right field shows the total austenite twin volume fraction, summed over all variants. The incident and output bars are depicted as solid, and colormapped by the axial stress in each of the panels. The specimen is depicted by spherical glyphs at the material points. The incoming compression pulse travels from lower-left to upper right in the figure. . . . . . . . . . . . . . . . . 120

6.32 SHPB simulation of an iron specimen for $\alpha-\varepsilon$ transformation at $1000 \mathrm{~m} / \mathrm{s}$. (Step 20.) The pulse reaches the specimen, which is already grossly deformed, with significant plasticity. Little transformation is seen. . . . . . . . . . . 121

6.33 SHPB simulation of an iron specimen for $\alpha-\varepsilon$ transformation at $1000 \mathrm{~m} / \mathrm{s}$. (Step 25.) The specimen is destroyed, and the results are clearly non-physical. 121

7.1 Simulated Voronoi microstruture with non-transforming inclusions (green) for anti-plane shear study. (Figure courtesy of D. Sun.) . . . . . . . . . . 127 


\section{Chapter 1}

\section{Introduction}

The mechanical behavior of materials is often the result of complex interactions between multiple microscopic deformation mechanisms. Two such mechanisms, present in a variety of polycrystalline materials, are martensitic phase transformation and plastic slip. In this thesis, we examine the behavior of solids where both these mechanisms are present and interact with each other. We do so in the two contexts: in Shape Memory Alloys (SMAs), and in iron under shock loading.

Shape-memory alloys display a number of interesting properties like the shape-memory effect and superelasticity. Shape-memory effect is the ability of the material to recover, on heating, deformation suffered below a critical temperature. Superelasticity is the ability of the material to recover strains beyond their apparent elastic limit. This is illustrated in the typical stress strain response shown as the solid curve in Figure 1.1. Superelasticity has proven to be extremely useful in a number of applications including implantable medical

devices, dental archwires, cell phone antennas and reading glasses. Nickel-Titanium or 


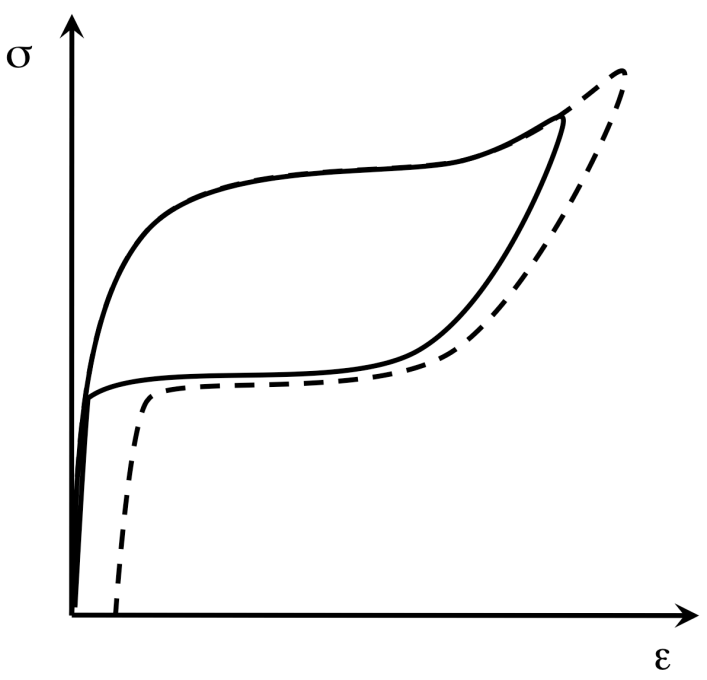

Figure 1.1: Typical stress strain response of a superelastc material. The superelastic response is shown in the solid curve. If the material is strained beyond a certain limit, one has incomplete memory as shown in the dashed curve

Nitinol has emerged as the material of choice, though a number of SMAs are known. We refer the reader to Otsuka and Wayman [67] and Yamauchi et al. [95] for a detailed overview.

The superelastic phenomenon has its limits, however. If the material is deformed beyond a certain point, it is unable to recover fully. Further, repeated loading can change the superelastic stress-strain curve. These can significantly affect applications and motivates the work in this thesis. Briefly, the shape-memory effect and superelasticity are manifestations of a martensitic phase transformation, while the loss of memory or superelasticity are manifestations of plasticity. Thus SMAs provide an important and useful context in understanding the interaction between these two phenomena. 


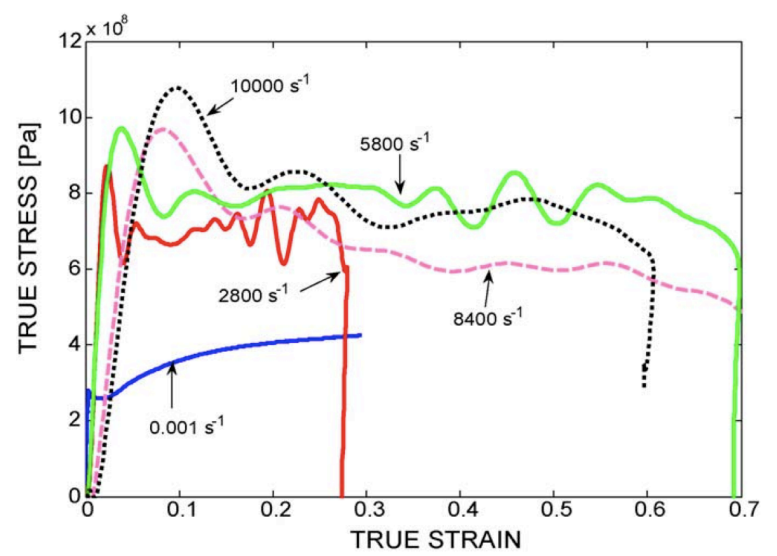

Figure 1.2: Response of pure iron at various strain rates. (From [76]. Reprinted with permission of the author.)

Iron and its alloys are also capable of martensitic phase transformations. In fact, much of the terminology used in this describing these phase transformations arise from the various phases of iron and steel. Interestingly the martensitic phase transformation in iron is almost always accompanied by plasticity. This interplay between plasticity and phase transformation has been exploited in various ways in tailoring the properties of steel including the recent work on TrIP steels $[22,96]$.

We focus in this thesis on another aspect of this interaction in iron. Figure 1.2 shows the experimental observations of [76] dynamic mechanical response of iron. These results were obtained in a split-Hopkinson bar using a shear-compression specimen. Note that the yield strength as well as hardening behavior depends sensitively on the rate of deformation. Further, it is believed that this behavior is related to the interplay between plasticity and phase transformation. 


\subsection{Deformation mechanisms and effects}

\subsubsection{Martensitic phase transformations}

Martensitic phase transformation is the distortion of a crystal lattice such that all of the constituent atoms cooperatively displace, with no change in long-range atomic order - a so-called diffusionless, first-order, solid-solid phase transformation from a high-symmetry, (usually) high-temperature austenite phase to a low-symmetry, (usually) low-temperature martensite phase. The austenite is shown schematically as the red square lattice while the martensite is shown schematically as the blue sheared lattice in Figure 1.3(a). Notice that

the loss of symmetry gives rise to multiple symmetry-related variants of martensite. Figure 1.3(b) shows the energy landscape resulting from such a phase transformation. Note that the energy is not convex, and this gives rise to fine scale microstructure and interesting behavior including superelasticity.

\subsubsection{Plastic slip}

Plastic slip occurs when one part of the crystal slides over another taking advantage of the periodic nature of crystals. Since the barrier for this sliding is very high, slip occurs through the motion of dislocations. Typically slip is restricted to a few (symmetry-related) slip systems consisting of a slip plane and a slip direction, and slip generally occurs when the local stress projected to the system exceeds some critical value. Since the crystal reconstructs after slip, slip is not recoverable. There is a very well-developed microscopic 


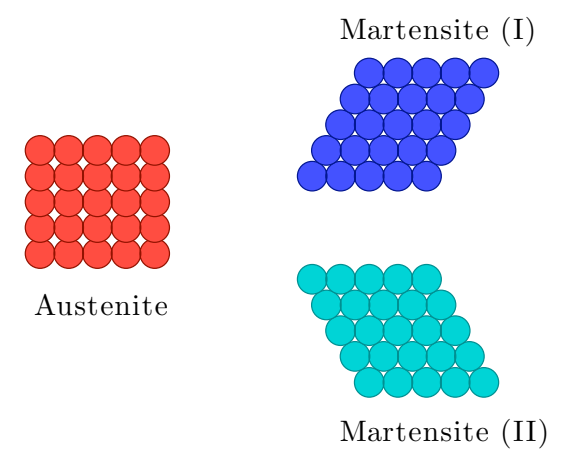

(a)

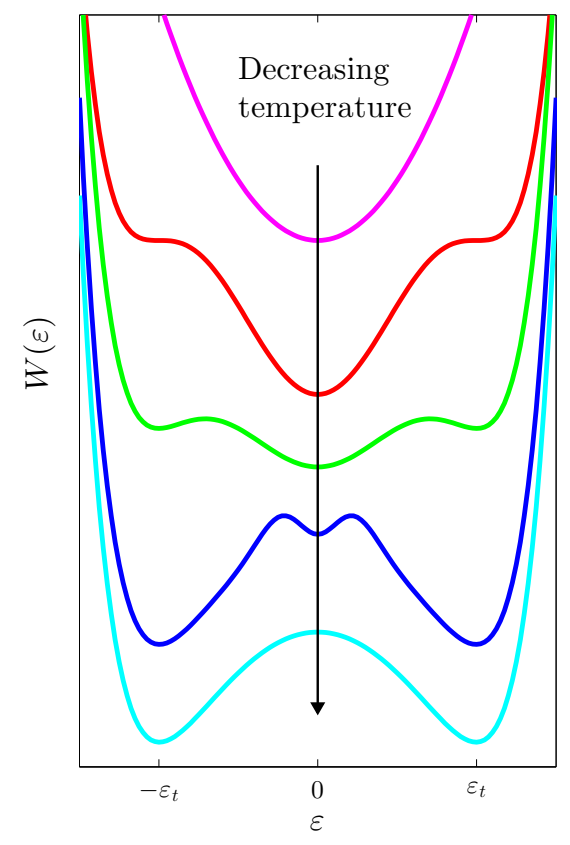

(b)

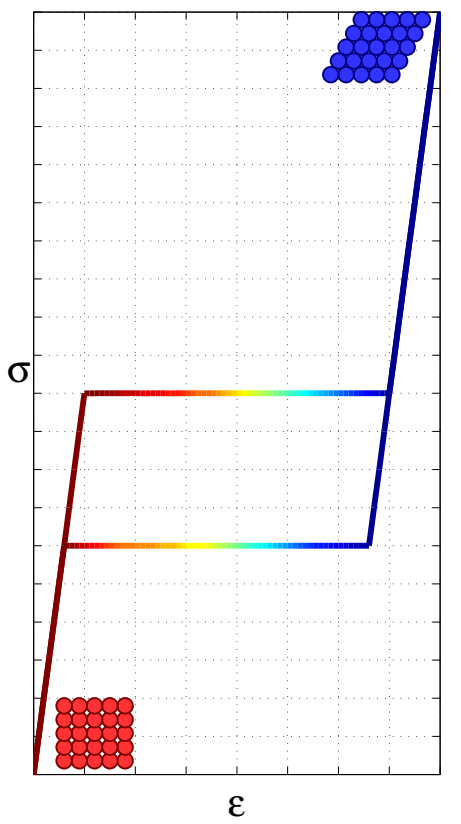

(c)

Figure 1.3: (a) Martensitic phase transformations. (b) Evolution of a non-convex energy potential as function of temperature (adapted from [27].) (c) Resulting stress-strain response of a single crystal associated with stress-stabilized martensite (from red or green curves in (b)). 


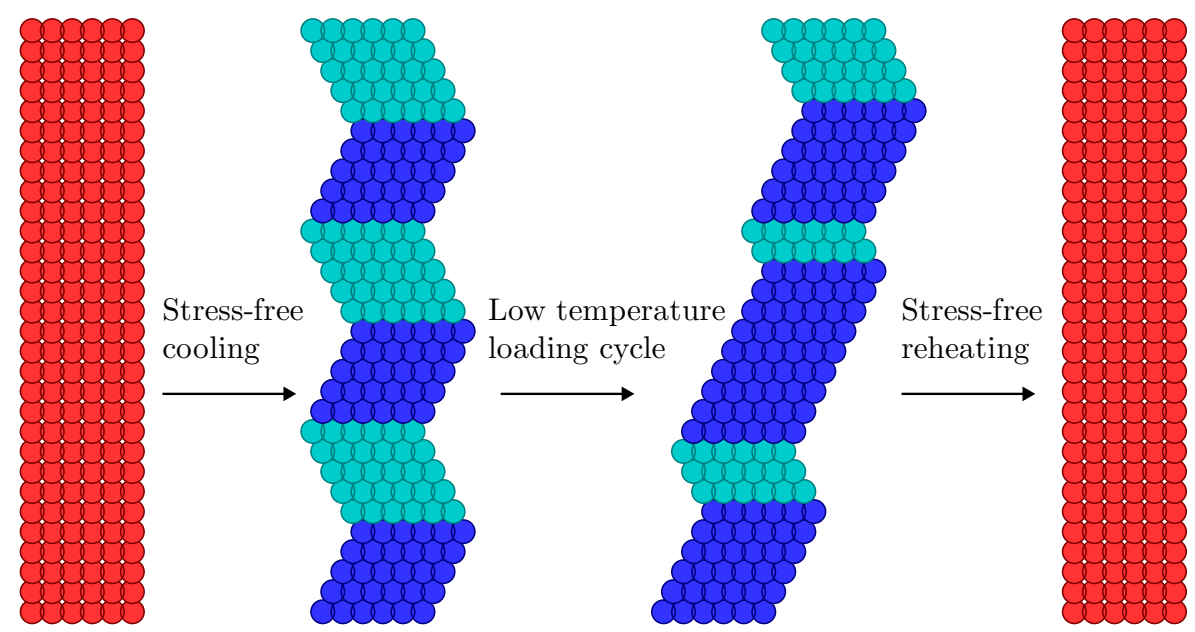

Figure 1.4: Schematic of the shape memory effect. At high termperature, the material is in the highly symmetric austenite phase (red). Upon cooling, mixtures of martensite (blue and cyan) form such that shape of the specimen, on average, remains undeformed. During loading cycles, the martensite variants reapportion themselves, leading to residual deformations after unloading. Upon reheating, all variants of martensite revert back to the same parent phase, recovering the apparent permanent deformations.

and macroscopic theory of plasticity. We refer the reader to $[35,70,54,62]$ for details.

\subsubsection{Shape memory and superelasticity}

The shape-memory effect and superelasticity are a manifestation of a martensitic phase transformation as illustrated in Figures 1.4 and 1.5. Superelasticity, also called pseudoelasticity, is observed when a material is above the transformation temperature and the martensite is "stress-induced" from the normally stable austenite. The transformation manifests itself in a yield-like behavior on the application of sufficient stress, and the recovery is the result of the reverse transformation. Often, the recovery is not perfect and there is some residual strain left after a deformation and recovery cycle. In fact, this hap- 


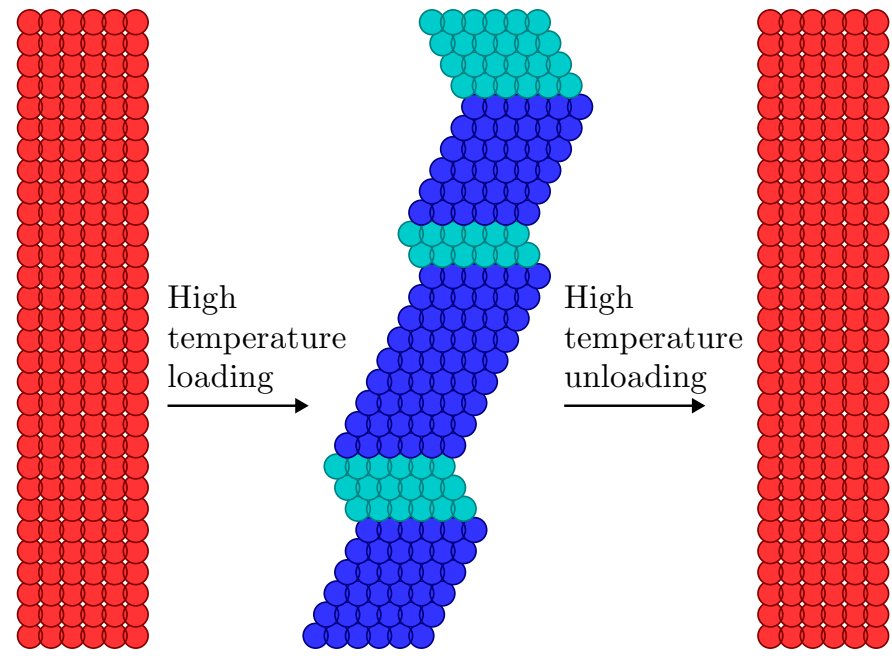

Figure 1.5: Schematic of superleasticity. At high temperatures, loading of the austenite phase can generate stress-stabilized martensite, which reduces the energy required to accomodate an applied deformation. The material returns to the austensite during unloading.

pens in every material if the deformation passes some limit. Further, the behavior of the material, and specifically the stress-strain curve, changes with cycling. At temperatures sufficiently above the transition temperature, the incomplete recovery and the evolution of the stress-strain behavior can be attributed to true plastic deformation in the material.

\subsection{Literature review}

Models of martensitic phase transformations are largely formulated at three length scales: micro-scale models, macro-scale models, and so-called meso-scale (or "micro-macro") models, which bridge the length scale between the previous two.

Micro-scale models, such as those pioneered by [9] and [3], usually have a strong foundation in the formal crystallographic theory of martensite (cf. [11]). Such models resolve the 
exact (sharp) interfaces between austenite and martensite variants, and have provided insight into phenomena such as nucleation, phase boundary propagation, and fine-scale twinning. Because of their computational complexity, however, micro-scale models are usually impractical for directly modelling macroscopic structures; the fine microstructures generated by slip (e.g. dislocation structures) and phase transformation become prohibitively expensive to compute.

Macro-scale models are typically phenomenological in nature, employing only macroscopic quantities as state variables. A somewhat exceptional case is that of [37], in which information about the microstructure is incorporated using the texture of the polycrystal as an evolving constraint on the macroscopically recoverable transformation strain. More traditional macro-scale models were developed to capture the 1-D mechanical response of SMAs, then generalized to 3-D ([71]; [86], [7], [8]; [18], [46], [68]). Some early models were shown to have difficulty capturing the response to nonproportional loading observed experimentally in [84], though advances have been made. In most of these models, an additive decomposition of the linearized strain into elastic and inelastic components is assumed, which is generalized to a multiplicative (Lee-Kroner) decomposition of the deformation gradient for finite kinematics in 3-D; a further additive decomposition of the inelastic strain rate tensor into transformation and reorientation parts is used in both the linearized and finite kinematics, which has been shown to successfully capture experimentally observed behavior. In general, these types of macro-scale models are the most amenable for commercial applications, such as FEM modelling of devices and structures. 
Perhaps more interesting are the investigations of SMA polycrystals at the grain-level, which are usually conducted using meso-scale models. Several early approaches focused on phase-field models with prescribed phenomenological laws for the free energy (e.g. [5]). Those of [49] and [48] employed polynomial expressions for the Ginzburg-Landau free energy, resulting in stress-strain relations which included a strain-softening regime; a similar (non-convex) Landau theory was developed in [4] around the same time, and was likewise used to study domain morphology in different temperature regimes. The detailed review by [40] analyzed two main types of polycrystal models: self-consistent type averaging of micromechanical models (so-called mean-field theories), and internal-variable phenomenological models. (Part I of that review, [69], also gives a very thorough description of the behavior and modelling of single-crystal SMAs.) The later are typically grounded in the formalism of [72], and largely based on plasticity-like formulations. A comparison of several such mesco-scale models (as well as some micro- and macro-scale models) to experimental results is given in [51].

It bears mentioning that many of the energy minimizing methods developed for early micro-scale models of SMAs, such as multi-well mixing ([9], [31]), have provided crucial insight in how to best account for micro-scale effects at larger length scales. In particular, since the quasi-convex minimization problem that arises from the micro-scale setting is difficult to compute for any general case, rigorous upper- and lower-bounds on the actual energies have been developed (e.g. [85]). These can be used as substitutes for the actual minimized energy, resulting in so-called relaxed potentials that can then be employed in 
meso- and macro-scale models.

A rigorous mathematical treatment of the influence of texture on the recoverable strains associated with the shape-memory effect in polycrystals (e.g. $[14,15,82])$ has also been conducted using formal homogenization theory. Comparison of numerical investigations of the recoverable strains to analytical bounds have proven insightful for studying the relation between single-crystalline and polycrystalline shape-memory behavior; such investigations may also be employed when non-linear homogenization problems become intractable analytically. In particular, we note the study of [89], as the FFT-based numerical method therein will be used as a starting point for the present study.

In [34], it was found that, for a particular setting with linearized kinematics, the upperbound given by a (Rank-1 convex) laminate model and the lower-bound given by simple convexification were surprisingly tight; the authors further noted that the actual solution might lie closer to the simple convex model than previously imagined. The convex model is analogous to a uniform stress assumption (Sachs bound) on the behavior of a mixture of martensite variants, and the Sachs bound was reported by [64] and [51] to agree reasonably well with experimental observations. A rationale for this result was given in [16], which explored the convex model in the anti-plane shear setting (for which it is exact, rather than merely a lower bound); this work serves to justify our use of convexified potentials in the chapters that follow.

While an emerging study of modelling martensite in polycrystalline SMAs continues to develop, the computational modelling of crystalline plasticity is much more firmly estab- 
lished (e.g. [83], chapter 9). The recent review by [78] describes the historical overview of the approach employed by most modern continuum-based computational crystal plasticity codes. Briefly, the kinematics of plasticity in single crystals are described by a multiplicative decomposition of the deformation gradient into elastic and plastic components; the time rate of change of the plastic component is assumed to be described, to first-order accuracy, by a summation of the contributions from the slip on each active system in the crystal. The evolution of slip on a system is governed by a prescribed kinetic relation, assumed to depend on a resolved (usually shear) stress on each system, relative to some critical value; hardening effects are incorporated by further introducing a phenomenological evolution equation for the critical value of the resolved stress.

The reviewers in [78] also make note of the similarity between the now classic approach to crystal plasticity and the emerging internal variable phenomenological models for martensite. The reviewers remark that, in the context of internal variable models, the finite element method is merely a variational solver for the underlying constitutive equations (and constraints), and that the addition of competing crystallographic deformation mechanisms (e.g. martensite formation) requires the formulation of local homogenization rules. The review goes on to describe the numerical simulations by [92] of a TrIP steel, in which a single grain of FCC austenite is embedded in a matrix of BCC ferrite, and the elastoplastic transformation process in the austenitic region is computed according to a prescribed plasticity-type model.

The modelling of TrIP steels in general poses an intriguing motivation to investigate 
the competition between plasticity and martensitic phase transformation. The most widely used models, such as those in [45] and [44], have proven to be reasonably successful at estimating the macroscopic response during creep tests, despite gross assumptions in the micromechanical underpinnings (cf. [90]); the model of [57] is in a similar vein. More recent atomistic simulations of nanowires have demonstrated good agreement with nano-scale experimental observations, but are impractical for modelling macro- or even meso-scale structures. It seems there is room for understanding the combined effects of plasticity and martensitic phase transformation between large and small length-scales to help formulate more accurate models for the polycrystalline behavior of these materials.

In general, large body of experimental work exists for the study of the evolution and recoverability of strains in phase transforming materials (cf. e.g. [19, 88, 55, 38], and references therein). We shall call attention to specific experimental observations and make comparisons to our own results in the chapters that follow.

\subsection{Chapter summary}

Both transformation and plasticity have origins at the atomistic/crystallographic level transformation occurs with a given transformation strain along habit planes, and plasticity occurs with given slip systems. Individually, their behavior across several length-scales has been well studied, and successful, physics-based constitutive models exist for crystalline

materials exhibiting either of these two phenomena. When transformation and slip are 
both active in a material, however, their interaction can be quite complex, especially in polycrystals where the grains are unaligned.

To account for this, we take a three-step approach in formulating our framework:

1. A meso-scale description is developed for the single-crystal response, based on energy relaxation methods described in [16].

2. Crystallographic and thermodynamic data are incorporated into the model in the form of grain orientation, strain of transformation, latent heat of transformation, slip systems, and standard thermo-elastic material properties.

3. The macroscopic response of a polycrystalline Representative Volume Element (RVE), whose grains obey the meso-scale description, is computed numerically.

We begin our development by reviewing the kinematics of martensitic phase transformation and plastic slip in Chapter 2 .

In Chapter 3, we develop a model for transformation and plasticity at the sub-granular level in the context of isothermal anti-plane shear, where the domain is two-dimensional and the deformation is a scalar. We use a meso-scale description for the transformation where we do not resolve the details of the martensitic microstructure but deal only with volume fractions of the different variants of martensite and that of the austenite (as in multivariant models [29]). We treat slip with crystal plasticity. This meso-scale treatment puts the two phenomena — transformation and plasticity — on par with each other. Further, it allows us to include crystallographic information while being computationally 
inexpensive. Similar to [89], we adapt the Fast Fourier Transform (FFT) based method of Michel et al. [59] to compute the response of the polycrystal.

We analyze the results of the anti-plane shear implementation in Chapter 4. We generate specimens with random microstructures, and subject them to various single- and multi-axial loading schemes. We also vary the relative ease (critical stress) of transformation and plasticity. We make various observations and compare them with experimental observations. The simplicity of the setting allows us to probe deeply into essential aspects with limited complications of extraneous issues. At the same time, the issues we study are generic and our results and insights carry forward to three dimensions.

In Chapter 5, we generalize the model to a 3-D setting with linearized kinematics, and demonstrate that for a fully implicit numerical scheme, the discrete time formulation of the framework is governed by a variational principle. We also propose an extension to finite kinematics.

In Chapter 6, we demonstrate a parallelized implementation of the generalized framework to model the $\alpha-\gamma$ and $\alpha-\varepsilon$ phase transitions in iron. We simulate a Taylor bar impact experiment, and demonstrate the computational scalability of the framework. We also propose a method to incorporate deformation twinning in the parent phase of the $\alpha-\varepsilon$ transformation, and show preliminary results from a simulated Split Hopkinson Pressure $\operatorname{Bar}(\mathrm{SHPB})$.

We summarize our work in Chapter 7, highlighting some key insights afforded by the modelling, and describe ongoing and future work. 
Lastly, it should be noted that Chapters $3 \& 4$ are a slightly expanded version of [74], and most passages have been lifted verbatim from this work. Chapters $5 \& 6$ will become the basis for a future publication [75]. 


\section{Chapter 2}

\section{Background}

\subsection{Continuum kinematics}

For completeness, and to establish our notation, we review the basic kinematics of a continuum. For a more thorough description, the reader is referred to various text books, including $[21,33,87]$. We consider a body $\Omega_{*}$ to be a collection of material points. For some reference configuration $\Omega_{0}$, each point in the body can be located in space by its position vector $\mathbf{X}$, relative to some origin $\mathbf{O}$. At some time $t$, the body is assumed to have evolved (i.e. deformed and displaced) to the current configuration $\Omega$, wherein each material point may now be located by the current position vector $\mathbf{x}$, given by the mapping $\mathbf{x}=\chi(\mathbf{X}, t)$. We use the standard abuse of notation and simply refer to $\mathbf{x}(\mathbf{X}, t)$ instead of $\chi$, and assume all quantities hereafter are functions of $(\mathbf{X}, t)$ unless explicitly stated otherwise. The displacement field, then, is given by $\mathbf{u}=\mathbf{X}-\mathbf{x}$. The deformation gradient is the

first derivative of the position vector, $\mathbf{F}=\frac{\partial \mathbf{x}}{\partial \mathbf{X}}=\nabla \mathbf{x}$, where $\nabla=\frac{\partial}{\partial \mathbf{X}}$. The displacement gradient $\mathbf{H}=\nabla \mathbf{u}$ is related to the deformation gradient by $\mathbf{H}=\frac{\partial \mathbf{u}}{\partial \mathbf{X}}=\frac{\partial(\mathbf{x}-\mathbf{X})}{\partial \mathbf{X}}=\mathbf{F}-\mathbf{I}$. 
For coherent deformations (no cracks, etc.), $\chi$ is invertible. We also assume that $J=$ $\operatorname{det}(\mathbf{F})>0$. Further, $\mathbf{F}$ has a polar decomposition $\mathbf{F}=\mathbf{R U}=\mathbf{V R}$, where $\mathbf{R} \in S O(3)$ is a proper orthogonal tensor (i.e. $\mathbf{R}^{-1}=\mathbf{R}^{T}$ and $\operatorname{det}(\mathbf{R})=1$ ) describing the rotation of the local material point, and unique $\mathbf{V}$ and $\mathbf{U}$ are positive definite symmetric matrices describing the stretch.

For future use, we define the Cauchy-Green deformation tensor as $\mathbf{C}=\mathbf{F}^{T} \mathbf{F}=\mathbf{U}^{2}$, and the Seth-Hill family of generalized strain measures $f_{(m)}$ (for integer order $m$ ) s.t.,

$$
\begin{aligned}
\mathbf{E}_{(m)} & =f_{(m)}(\mathbf{C}) \\
& =\left\{\begin{array}{ll}
\frac{1}{m}\left(\mathbf{C}^{\frac{m}{2}}-\mathbf{I}\right), & m \neq 0 \\
\frac{1}{2} \ln (\mathbf{C}), & m=0
\end{array} .\right.
\end{aligned}
$$

For $m=2$, we recover the Green-Lagrange strain $\mathbf{E}_{(2)}=\frac{1}{2}(\mathbf{C}-\mathbf{I})$; for $m=-2$, we recover the Almansi strain $\mathbf{E}_{(-2)}(\mathbf{x}, t)=\frac{1}{2}(\mathbf{I}-\mathbf{b})$, where $\mathbf{b}(\mathbf{x}, t)=\mathbf{C}^{-1}(\mathbf{x}, t)=\mathbf{F}^{-1} \mathbf{F}^{-T}$; and for $m=1$, we recover the Biot strain $\mathbf{E}_{(1)}=(\mathbf{U}-\mathbf{I})$. The $m=0$ case, sometimes called the Hencky strain $\mathbf{E}_{(0)}=\frac{1}{2} \ln (\mathbf{C})$, has some special properties discussed in Chapter 5 .

We note that the Green-Lagrange strain is a nonlinear measure of the displacement gradient; by substitution, $\mathbf{E}_{(2)}=\frac{1}{2}(\mathbf{C}-\mathbf{I})=\frac{1}{2}\left(\mathbf{F}^{T} \mathbf{F}-\mathbf{I}\right)=\frac{1}{2}\left((\nabla \mathbf{u}+\mathbf{I})^{T}(\nabla \mathbf{u}+\mathbf{I})-\mathbf{I}\right)=$ $\frac{1}{2}\left((\nabla \mathbf{u})^{T}+\nabla \mathbf{u}+(\nabla \mathbf{u})^{T} \nabla \mathbf{u}\right)$, where we see the nonlinear term $(\nabla \mathbf{u})^{T} \nabla \mathbf{u}$. If we linearize this expression, or indeed any of the strain measures, we get $\varepsilon=\frac{1}{2}\left((\nabla \mathbf{u})^{T}+\nabla \mathbf{u}\right)$, which is a justifiable approximation to the strain when $\nabla \mathbf{u}$ is small (s.t. $(\nabla \mathbf{u})^{T} \nabla \mathbf{u}$ is vanishingly 
small).

We further note that under a pure rigid body rotation, $\mathbf{U}=\mathbf{V}=\mathbf{I}$ and $\mathbf{F}=\mathbf{R}$. We expect no strain associated with this "deformation," and indeed, for each of the nonlinear strain measures, we see $\mathbf{C}=\mathbf{F}^{T} \mathbf{F}=\mathbf{R}^{T} \mathbf{R}=\mathbf{I}$, leading to $\mathbf{E}_{(m)}=\frac{1}{m}\left(\mathbf{I}^{\frac{m}{2}}-\mathbf{I}\right)=\mathbf{0}$ (and $\mathbf{E}_{(0)}=\frac{1}{2} \ln (\mathbf{I})=\mathbf{0}$ for the $m=0$ case $)$. However, for the linearized strain, $\varepsilon=\frac{1}{2}\left(\mathbf{R}^{T}+\mathbf{R}\right)-\mathbf{I}$ does not vanish. We see then that the linearized strain measure fails to properly distinguish rotations from strains. Additional troubles with partitioning of rotations will be discussed in Chapter 5.

Finally we look at the time derivative of $\mathbf{F}$,

$$
\begin{aligned}
\dot{\mathbf{F}} & =\frac{\partial \mathbf{F}}{\partial t} \\
& =\frac{\partial}{\partial t}\left(\frac{\partial \mathbf{x}}{\partial \mathbf{X}}\right) \\
& =\frac{\partial}{\partial \mathbf{X}}\left(\frac{\partial \mathbf{x}}{\partial t}\right)
\end{aligned}
$$

Defining the velocity $\mathbf{V}(\mathbf{X}, t)=\frac{\partial \mathbf{x}(\mathbf{X}, t)}{\partial \mathbf{X}}=\mathbf{v}(\mathbf{x}(\mathbf{X}, t), t)$ (and acknowledging that we have reused/redefined the variable $\mathbf{V}$ ), we have

$$
\begin{aligned}
\dot{\mathbf{F}} & =\frac{\partial}{\partial \mathbf{X}}(\mathbf{V}(\mathbf{X}, t)) \\
& =\frac{\partial}{\partial \mathbf{x}}(\mathbf{v}(\mathbf{x}, t)) \cdot \frac{\partial \mathbf{x}}{\partial \mathbf{X}} \\
& =\mathbf{L} \cdot \mathbf{F}
\end{aligned}
$$


where $\mathbf{L}(\mathbf{x}, t)$ is the spatial velocity gradient. The symmetric portion of the velocity gra-

dient is called the rate of deformation tensor, $\mathbf{D}(\mathbf{x}, t)=\frac{1}{2}\left(\mathbf{L}+\mathbf{L}^{T}\right)$; the antisymmetric portion is called the spin tensor, $\mathbf{W}(\mathbf{x}, t)=\frac{1}{2}\left(\mathbf{L}-\mathbf{L}^{T}\right)$.

\subsection{Crystallography of martensite}

We review the crystallographic theory of martensitic phase transformation, specifically, the mathematical description of the associated lattice distortions. We refer to [11] for further details. We assume that, at some (usually) high temperature, the atomic arrangement of a material adopts a high symmetry lattice configuration which we call the austenite phase. At some (usually) lower temperature, this configuration becomes unstable, as the material can reduce its overall energy by adopting a lower symmetry lattice configuration, which we call martensite. We consider only cases where this change in structure is essentially diffusionless.

In general, the atomic skeletal lattice of a single crystal may be described as a Bravais lattice, with basis vectors $\left\{\mathbf{e}_{1}, \mathbf{e}_{2}, \mathbf{e}_{3}\right\}$. We begin by assuming that a single-crystal starts in a uniform (single-domain) austenite phase, such that these basis vectors describe the high symmetry lattice. We introduce a second set of basis vectors, $\left\{\mathbf{f}_{1}, \mathbf{f}_{2}, \mathbf{f}_{3}\right\}$ to describe the lattice of a single domain of martensite. Since the martensitic phase transformation is 


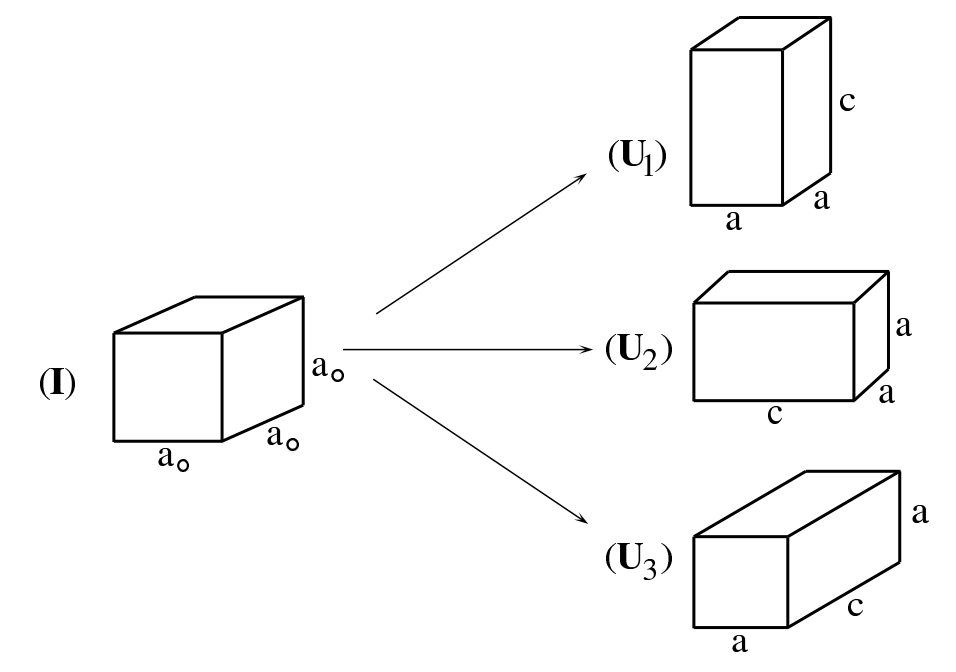

Austenite

Martensite

Figure 2.1: Variants of martensite in a cubic to tetragonal transformation. (From [11]. Reprinted with permission from author.)

diffusionless, these basis vectors are related by a linear mapping,

$$
\mathbf{f}_{k}=\mathbf{F e}_{k}
$$

The polar decomposition of $\mathbf{F}$ gives a unique, positive definite matrix $\mathbf{U}$ known as the Bain matrix.

The symmetry of the parent phase implies the existence of a set of rotations $\mathbf{R}_{i}$ that map the austenite lattice back onto itself (i.e. the point-group of the austenite, $\mathcal{P}_{a}$ ). This leads to a set of symmetry related variants of martensite (see Figure 2.1),

$$
\mathbf{U}_{i}=\mathbf{R}_{i}^{T} \mathbf{U R}_{i}, \quad \forall \mathbf{R}_{i} \in \mathcal{P}_{a}
$$


We note that those $\mathbf{R}_{i}$ which also belong to the point group of the martensite $\mathcal{P}_{m}$ do not each produce a new variant of martensite (i.e. some variants might be defined redundantly due to symmetries of the martensite phase). In general, the number of unique martensitic variants is given by $\frac{P_{a}}{P_{m}}$, the ratio of the number of unique members (i.e. the cardinality) of the austenite and martensite point groups, respectively.

Even though the lattice vectors give an atomistic description, we invoke the CauchyBorn hypothesis and assume that these vectors behave like material filaments, which deform according to the (continuum theory) deformation gradient $\mathbf{F}$.

The principle of material frame indifference for the Helmholtz free energy density of the lattice states,

$$
W(\mathbf{Q F})=W(\mathbf{F}), \quad \forall \mathbf{Q} \in S O(3)
$$

This implies that,

$$
W(\mathbf{F})=W(\mathbf{R U})=W(\mathbf{U})
$$

Further, material symmetry implies,

$$
W(\mathbf{F})=W\left(\mathbf{F} \mathbf{R}_{i}\right), \quad \forall \mathbf{R}_{i} \in \mathcal{P}_{a}
$$




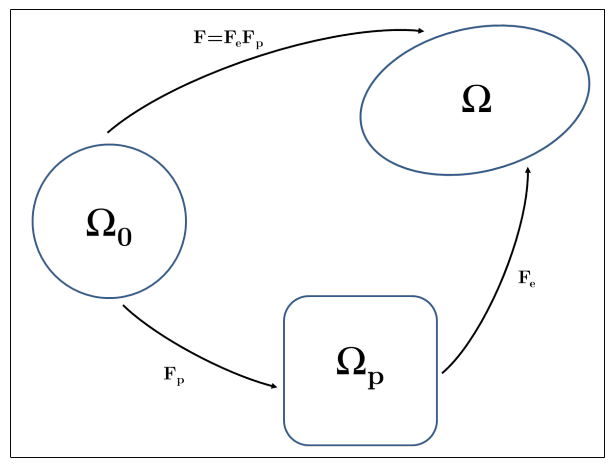

Figure 2.2: Stress-free intermediate configuration associated with plasticity.

This implies that,

$$
W\left(\mathbf{U}_{i}\right)=W\left(\mathbf{R}_{i}^{T} \mathbf{U R}_{i}\right), \quad \forall \mathbf{R}_{i} \in \mathcal{P}_{a}
$$

From Eq. (2.10) we conclude that the energy associated with each variant of martensite must be equal, and from Eq. (2.8), we conclude that each variant is a well in the energy landscape.

Since each $\mathbf{U}_{i}$ is symmetric positive-definite, the linearized transformation strain $\varepsilon_{i}^{t}$ associated with each variant is given simply by $\varepsilon_{i}^{t}=\mathbf{U}_{i}-\mathbf{I}$.

\subsection{Crystal plasticity}

We now look at the description of an elastic-plastic single crystal. We refer the reader to $[54,62]$ for further details. Given some deformed configuration $\Omega$ associated with $\mathbf{F}$ relative to $\Omega_{0}$, we postulate stress-free configuration $\Omega_{p}$ associated with $\mathbf{F}_{p}$. We define the relative 
displacement as $\mathbf{F}_{e}=\mathbf{F} \mathbf{F}_{p}^{-1}$ so that $\mathbf{F}_{p}=\mathbf{F}_{e}^{-1} \mathbf{F}$. We postulate that the deformation is elastic. This multiplicative decomposition of the deformation gradient is known as the Lee-Kroner decomposition.

From the chain rule and (2.4),

$$
\dot{\mathbf{F}}=\dot{\mathbf{F}}_{e} \mathbf{F}_{p}+\mathbf{F}_{e} \dot{\mathbf{F}}_{p}
$$

Consequently,

$$
\begin{aligned}
\mathbf{L} & =\left(\dot{\mathbf{F}}_{e} \mathbf{F}_{p}+\mathbf{F}_{e} \dot{\mathbf{F}}_{p}\right) \mathbf{F}_{p}^{-1} \mathbf{F}_{e}^{-1} \\
& =\dot{\mathbf{F}}_{e} \mathbf{F}_{e}^{-1}+\mathbf{F}_{e}\left(\dot{\mathbf{F}}_{p} \mathbf{F}_{p}\right) \mathbf{F}_{e}^{-1} \\
& =\mathbf{L}_{e}+\mathbf{F}_{e}\left(\mathbf{L}_{p}\right) \mathbf{F}_{e}^{-1}
\end{aligned}
$$

where $\mathbf{L}$ is the spatial velocity gradient in the current configuration relative to the reference configuration, $\mathbf{L}_{e}$ is velocity gradient in the current configuration relative to the plastic configuration, and $\mathbf{L}_{p}$ is the spatial velocity gradient in the plastic configuration relative to the reference configuration. For deformations due to simple dislocation slip (and where the first-order approximation of $\mathbf{F}_{p}$ is reasonable), the plastic velocity gradient is the sum of the shearing rate on each active slip system [73], so,

$$
\mathbf{L}_{p}=\sum_{\alpha}^{M} \dot{\gamma}_{\alpha}\left(\mathbf{s}_{\alpha} \otimes \mathbf{m}_{\alpha}\right)
$$


where $\dot{\gamma}_{\alpha}$ is the scalar shearing rate associated with the $\alpha$ slip system, $\mathbf{s}_{\alpha}$ is a unit vector in the direction of the slip, and $\mathbf{m}_{\alpha}$ is the unit normal to the plane of the slip, and $M$ is the number of slip systems. Above, $\mathbf{s}_{\alpha}$ and $\mathbf{m}_{\alpha}$ are defined in the reference configuration, and $\mathbf{s}_{\alpha} \cdot \mathbf{m}_{\alpha}=0$, so that the slip is a pure shear. 


\section{Chapter 3}

\section{Antiplane Shear Model}

\section{$3.1 \quad$ Kinematics}

We turn our attention to setting of antiplane shear. We envision a state of strain generated by out-of-plane displacements, i.e.,

$$
\mathbf{u}=\left(\begin{array}{c}
0 \\
0 \\
u_{3}
\end{array}\right), \quad u_{3}=u_{3}\left(X_{1}, X_{2}\right)
$$

We see that the displacement gradient has only two nonzero components,

$$
\nabla \mathbf{u}=\left(\begin{array}{ccc}
0 & 0 & \frac{\partial u_{3}}{\partial X_{1}} \\
0 & 0 & \frac{\partial u_{3}}{\partial X_{2}} \\
0 & 0 & 0
\end{array}\right)
$$


Recognizing that the entire state of deformation depends solely on the scalar function $u_{3}\left(X_{1}, X_{2}\right)$, we define a reduced strain vector,

$$
\varepsilon=\nabla u_{3}=\left(\begin{array}{l}
\varepsilon_{1} \\
\varepsilon_{2}
\end{array}\right)
$$

where $\varepsilon_{1}=\partial u_{3} / \partial X_{1}=2 \varepsilon_{31}$, and $\varepsilon_{2}=\partial u_{3} / \partial X_{2}=2 \varepsilon_{32}$. We drop the reference subscript $S 3$ in the rest of Chapters $3 \& 4$, and write $u=u_{3}\left(X_{1} X_{2}\right)$. The stress in this setting has at most two independent components, and we define the analogous stress vector,

$$
\sigma=\left(\begin{array}{c}
\sigma_{1} \\
\sigma_{2}
\end{array}\right)
$$

where $\sigma_{1}=\sigma_{31}$, and $\sigma_{2}=\sigma_{32}$.

In this special setting, we see that the deformation gradient $\mathbf{F}$ is commutative. Let $\varepsilon^{a}=\varepsilon_{1}^{a} \hat{\mathbf{e}}_{1}+\varepsilon_{2}^{a} \hat{\mathbf{e}}_{2}$ and $\varepsilon^{b}=\varepsilon_{1}^{b} \hat{\mathbf{e}}_{1}+\varepsilon_{2}^{b} \hat{\mathbf{e}}_{2}$, such that for two anti-plane shear deformation gradients $\mathbf{F}^{a}$ and $\mathbf{F}^{b}$

$$
\begin{aligned}
\mathbf{F}^{a} & =\mathbf{I}+\varepsilon^{a} \otimes \hat{\mathbf{e}}_{3} \\
\mathbf{F}^{b} & =\mathbf{I}+\varepsilon^{b} \otimes \hat{\mathbf{e}}_{3} \\
\mathbf{F}^{a} \mathbf{F}^{b} & =\mathbf{I}+\left(\varepsilon^{a}+\varepsilon^{b}\right) \otimes \hat{\mathbf{e}}_{3}
\end{aligned}
$$

since $\varepsilon^{a} \cdot \hat{\mathbf{e}}_{3}=\varepsilon^{b} \cdot \hat{\mathbf{e}}_{3}=0$. From Eqns. (2.1) and (3.7), this leads to a truly additive 
decomposition of Green-Lagrange strain measure.

For an elastic-plastic material which undergoes martensitic phase transformation, we assume that the strain can be linearly decomposed into three components,

$$
\varepsilon=\varepsilon^{e}+\varepsilon^{t}+\varepsilon^{p}
$$

where $\varepsilon^{e}$ is the elastic strain, $\varepsilon^{t}$ is the transformation strain and $\varepsilon^{p}$ is the plastic strain.

At the length-scale of interest, the transformation strain is itself the average over complex microstructures. Therefore, we assume that it can be further decomposed as

$$
\varepsilon^{t}=\sum_{i=1}^{N} \lambda_{i} \varepsilon_{i}^{t}
$$

where $\lambda_{i}$ and $\varepsilon_{i}^{t}$ are the volume fraction and stress-free strain of the $i$ th (correspondence) variant of martensite respectively. $\lambda_{0}$ is the volume fraction of austenite and we take the stress-free strain of the austenite to be zero by choice of reference configuration. The volume fractions satisfy the constraint

$$
\lambda_{i} \in[0,1], \quad \sum_{i=1}^{N} \lambda_{i} \leq 1, \quad \lambda_{0}=1-\sum_{i=1}^{N} \lambda_{i} .
$$

Similarly, the plastic strain $\varepsilon^{p}$ may be decomposed to be the sum of the contribution 
from each of the $M$ slip systems,

$$
\varepsilon^{p}=\sum_{\alpha=1}^{M} \gamma_{\alpha} \varepsilon_{\alpha}^{p}
$$

where $\varepsilon_{\alpha}^{p}$ is the strain (direction) associated with the $\alpha$ th slip system.

We regard the volume fractions $\lambda_{i}$ and the slip activities $\gamma_{\alpha}$ to be internal variables.

\subsection{Balance laws}

We assume the usual balance laws of continuum mechanics. The balance of linear momentum states

$$
\nabla \cdot \sigma+b=0
$$

where $\sigma$ denotes the stress and $b$ the body force, and we neglect inertia. The balance of angular momentum is automatic in antiplane shear. The balance of energy states

$$
\dot{\epsilon}=\sigma \cdot \dot{\varepsilon}-\nabla \cdot q+r
$$

where $\epsilon$ is the internal energy per unit volume, $q$ is the heat flux and $r$ is the radiative heating. The second-law of thermodynamics is formulated as the Claussius-Duhem inequality,

$$
\dot{\mathcal{D}}:=\sigma \cdot \dot{\varepsilon}-\dot{\epsilon}+\theta \dot{\eta}-\frac{\nabla \theta}{\theta} \cdot q \geq 0
$$


where $\dot{\mathcal{D}}$ is the rate of dissipation per unit volume, $\eta$ is the entropy density, and $\theta$ is the absolute temperature. Introducing the Helmholtz free energy density $W=\epsilon-\eta \theta$, we may rewrite the Claussius-Duhem inequality as

$$
\dot{\mathcal{D}}=\sigma \cdot \dot{\varepsilon}-\dot{W}-\eta \dot{\theta}-\frac{\nabla \theta}{\theta} \cdot q \geq 0
$$

\subsection{Constitutive framework}

We assume that the free energy density, stress and entropy are functions of the strain, the volume fractions, slip activities, temperature as well as the material point:

$$
W=W\left(\varepsilon, \lambda_{i}, \gamma_{\alpha}, \theta, x\right), \quad \sigma=\sigma\left(\varepsilon, \lambda_{i}, \gamma_{\alpha}, \theta, x\right), \quad \eta=\eta\left(\varepsilon, \lambda_{i}, \gamma_{\alpha}, \theta, x\right)
$$

Substituting these in (3.15), we conclude

$$
\dot{\mathcal{D}}=\sigma \cdot \dot{\varepsilon}-\frac{\partial W}{\partial \varepsilon} \cdot \dot{\varepsilon}-\sum_{i=0}^{N} \frac{\partial W}{\partial \lambda_{i}} \dot{\lambda}_{i}-\sum_{\alpha=1}^{M} \frac{\partial W}{\partial \gamma_{\alpha}} \dot{\gamma}_{\alpha}-\frac{\partial W}{\partial \theta} \dot{\theta}-\eta \dot{\theta}-\frac{\nabla \theta}{\theta} \cdot \dot{q}
$$

Arguing as in Coleman and Noll [23], we conclude

$$
\begin{aligned}
& \sigma=\frac{\partial W}{\partial \varepsilon}, \\
& \eta=-\frac{\partial W}{\partial \theta}
\end{aligned}
$$


Further, following Rice [73] and Abeyaratne and Knowles [2], we identify the thermodynamic driving forces for the kinetic or internal variables,

$$
\begin{aligned}
& d_{i}^{\lambda}=-\frac{\partial W}{\partial \lambda_{i}} \\
& d_{\alpha}^{\gamma}=-\frac{\partial W}{\partial \gamma_{\alpha}}
\end{aligned}
$$

and postulate that they evolve according to kinetic relations

$$
\begin{aligned}
& \dot{\lambda}_{i}=f_{i}\left(d_{i}^{\lambda}\right), \\
& \dot{\gamma}_{\alpha}=g_{\alpha}\left(d_{\alpha}^{\gamma}\right) .
\end{aligned}
$$

The functions $f_{i}, g_{\alpha}$ satisfy the relations

$$
f_{i}\left(d_{i}^{\lambda}\right) d_{i}^{\lambda} \geq 0, \quad g_{\alpha}\left(d_{\alpha}^{\gamma}\right) d_{\alpha}^{\gamma} \geq 0
$$

consistent with the Claussius-Duhem inequality. Finally we assume Fourier's law of heat conduction or

$$
q=-K \nabla \theta
$$

for positive definite $K$. 


\subsection{Isothermal setting and constitutive law}

The considerations above provide us the governing equations. Given initial conditions, we solve for the evolution of the displacement field, the transformation strains at each position, the plastic activity at each point and the temperature field over a period of time by simultaneously solving the equilibrium equation (3.12), the evolution equations $(3.22$, 3.23) and the energy balance (3.13).

We now specialize to the isothermal setting in the absence of body forces. The governing equations now are the equilibrium equation (3.12), and the evolution equations (3.22, 3.23).

In the rest of the Chapters $3 \& 4$, we work at a fixed temperature with the specific free energy of the form

$$
W=\frac{1}{2}\left(\varepsilon-\varepsilon^{t}-\varepsilon^{p}\right) \cdot C \cdot\left(\varepsilon-\varepsilon^{t}-\varepsilon^{p}\right)+w \sum_{i=1}^{N} \lambda_{i}
$$

where $w$ is the chemical free energy, $C$ is the elastic modulus assumed to be equal in both phases, and $\varepsilon^{t}$ and $\varepsilon^{p}$ are given by (3.9) and (3.11) respectively. In a heterogenous material as we are considering here, $C, \varepsilon_{i}^{t}$ and $\varepsilon_{\alpha}^{p}$ depend on position $x$. Substituting into (3.27), (3.20), (3.21), the stress and driving forces associated with the chosen constitutive law 
become

$$
\begin{aligned}
\sigma & =C \cdot\left(\varepsilon-\varepsilon_{t}-\varepsilon_{p}\right), \\
d_{i}^{\lambda} & =\sigma \cdot \varepsilon_{i}^{t}-w, \\
d_{\alpha}^{\gamma} & =\sigma \cdot \varepsilon_{\alpha}^{p} .
\end{aligned}
$$

We assume the kinetic relations

$$
\begin{aligned}
& \dot{\lambda}_{i}= \begin{cases}h_{t}\left(d_{i}^{\lambda}-d_{c}\right) & d_{i}^{\lambda} \geq d_{c}, \lambda_{i} \geq 0, \sum_{i=1}^{N} \lambda_{i} \leq 1, \\
0 & \text { else, } \\
h_{t}\left(d_{i}^{\lambda}+d_{c}\right) & d_{i}^{\lambda} \leq-d_{c}, \lambda_{i} \geq 0, \sum_{i=1}^{N} \lambda_{i} \leq 1\end{cases} \\
& \dot{\gamma}_{\alpha}= \begin{cases}h_{p}\left(d_{\alpha}^{\gamma}-\tau_{c}\right) & d_{\alpha}^{\gamma} \geq \tau_{c}, \\
0 & \text { else, } \\
h_{p}\left(d_{\alpha}^{\gamma}+\tau_{c}\right) & d_{\alpha}^{\gamma} \leq-\tau_{c}\end{cases}
\end{aligned}
$$

where $d_{c}$ is the critical driving force for transformation, $\frac{1}{h_{t}}$ is the transformation rate sensitivity coefficient (viscosity-like parameter), $\tau_{c}$ is the critical resolved stress for plasticity, $\frac{1}{h_{p}}$ is the plastic rate sensitivity coefficient (viscosity-like parameter). 


\subsection{Staggered update and FFT Solver}

We use a staggered implicit-explicit time discretization to obtain

$$
\begin{aligned}
& \nabla \cdot \sigma\left(\varepsilon^{n+1},\left\{\lambda_{i}^{n}\right\},\left\{\gamma_{\alpha}^{n}\right\}\right)=0, \\
& \frac{\lambda_{i}^{n+1}-\lambda_{i}^{n}}{\Delta t}=f_{i}\left(d_{i}^{\lambda}\left(\varepsilon^{n+1},\left\{\lambda_{i}^{n}\right\},\left\{\gamma_{\alpha}^{n}\right\}\right)\right), \\
& \frac{\gamma_{\alpha}^{n+1}-\gamma_{\alpha}^{n}}{\Delta t}=g_{\alpha}\left(d_{\alpha}^{\gamma}\left(\varepsilon^{n+1},\left\{\lambda_{i}^{n}\right\},\left\{\gamma_{\alpha}^{n}\right\}\right)\right),
\end{aligned}
$$

which are solved successively. With the staggered update, we see that in Eq. (3.32), we are solving for mechanical equilibrium of an elastic medium with fixed eigenstrains $\varepsilon_{t}$ and $\varepsilon_{p}$ (determined by $\left\{\lambda_{i}^{n}\right\},\left\{\gamma_{\alpha}^{n}\right\}$ ). This can be solved efficiently with an iterative Fast Fourier Transform solver following [41, 89].

Recalling (3.27), we rewrite (3.32) as

$$
\begin{aligned}
& \nabla \cdot s=0 \\
& \text { where }\left\{\begin{array}{l}
s=C\left(\cdot \nabla u-\varepsilon_{t}-\varepsilon_{p}\right) \\
u=E \cdot x+v, \quad \text { with } v \# \text { (periodic) } \\
\langle\nabla u\rangle=E
\end{array}\right.
\end{aligned}
$$

where we have decomposed $\nabla u=E+\nabla v$ into its spatial average $E=\langle\nabla u\rangle$ and a mean-free

periodic field $\nabla v(\langle\nabla v\rangle=0$ by periodicity). (This decomposition will be necessary since 
the non-trivial solution for the Laplacian operator under periodic boundary conditions is only unique up to a constant, i.e. the mean strain.) We introduce a uniform stiffness matrix $C^{0}$, and for simplicity, choose $C^{0}$ to be the scaled idenity matrix $C^{0}=\mu^{0} I$. (So long as it is uniform in space, the choice of $C^{0}$ does not affect the final solution, but will affect the rate of convergence of the method). We then have

$$
\begin{aligned}
& \nabla \cdot\left(C \cdot\left(E+\nabla v-\varepsilon_{t}-\varepsilon_{p}\right)\right)=0, \\
\Longleftrightarrow & \nabla \cdot(C \cdot \nabla v)=-\nabla \cdot\left(C \cdot\left(E-\varepsilon_{t}-\varepsilon_{p}\right)\right), \\
\Longleftrightarrow & \nabla \cdot\left(\left(C-C^{0}\right) \cdot \nabla v+C^{0} \cdot \nabla v\right)=-\nabla \cdot\left(C \cdot\left(E-\varepsilon_{t}-\varepsilon_{p}\right)\right), \\
\Longleftrightarrow & \nabla \cdot\left(C^{0} \cdot \nabla v\right)=-\nabla \cdot\left(\left(C^{0}-C\right) \cdot \nabla v+C \cdot\left(E-\varepsilon_{t}-\varepsilon_{p}\right)\right)
\end{aligned}
$$

We see that the last equation is the basis for a recursive alogrithm, which can be solved efficiently in the Fourier domain. Assuming an initial guess $v^{\text {old }}$, we solve for $v$. Letting 
$f=\left(C^{0}-C\right) \cdot \nabla v^{\text {old }}+C \cdot\left(E-\varepsilon_{t}-\varepsilon_{p}\right)$, then,

$$
\begin{aligned}
& \nabla \cdot\left(C^{0} \cdot \nabla v\right)=-\nabla \cdot\left(\left(C^{0}-C\right) \cdot \nabla v^{\text {old }}+C \cdot\left[E-\varepsilon_{t}-\varepsilon_{p}\right]\right) \\
& \Longleftrightarrow \nabla \cdot\left(C^{0} \cdot \nabla v\right)=-\nabla \cdot f \\
& \Longleftrightarrow \mathcal{F F} \mathcal{T}\left(\nabla \cdot\left(C^{0} \cdot \nabla v\right)\right)=\mathcal{F F} \mathcal{T}(-\nabla \cdot f), \\
& \Longleftrightarrow(-i k) \cdot C^{0} \cdot(-i k) \hat{v}=-(-i k) \cdot \hat{f}, \\
& \Longleftrightarrow i k \cdot C^{0} \cdot i k \hat{v}=i k \cdot \hat{f}, \\
& \Longleftrightarrow k \cdot C^{0} \cdot k \hat{v}=-i k \cdot \hat{f}, \\
& \Longleftrightarrow \hat{v}=-i\left(k \cdot C^{0} \cdot k\right)^{-1} k \cdot \hat{f}, \\
& \Longleftrightarrow(-i k) \hat{v}=(-i k) \otimes(-i)\left(k \cdot C^{0} \cdot k\right)^{-1} k \cdot \hat{f}, \\
& \Longleftrightarrow(-i k) \hat{v}=-k \otimes\left(k \cdot C^{0} \cdot k\right)^{-1} k \cdot \hat{f}, \\
& \Longleftrightarrow(-i k) \hat{v}=-\frac{k \otimes k}{\mu^{0}|k|^{2}} \cdot \hat{f}, k \neq 0, \\
& \Longleftrightarrow(-i k) \hat{v}=-\hat{\Gamma}^{0} \cdot \hat{f} \\
& \Longleftrightarrow \mathcal{I F} \mathcal{F} \mathcal{T}((-i k) \hat{v})=\mathcal{I} \mathcal{F} \mathcal{F} \mathcal{T}\left(-\hat{\Gamma}^{0} \cdot \hat{f}\right) \\
& \Longleftrightarrow \nabla v=-\Gamma^{0} * f
\end{aligned}
$$

where $i=\sqrt{-1}$ and $k$ is the frequency vector associated with the Fourier transform $x \rightarrow k$, i.e. $f\left(x_{1}, x_{2}\right) \rightarrow \hat{f}\left(k_{1}, k_{2}\right)$.

We see that, in the Fourier domain, the Green's function for the assumed uniform 
reference medium represented by $C^{0}$ has a very simple form, essentially, $\hat{\Gamma}^{0}=\frac{k \otimes k}{|k|^{2}}$. Also, note that for $k=0$, the value is undefined, but we know that the first term in the Fourier expansion corresponds precisely to the mean value, so for $v(x) \rightarrow \hat{v}(k), \hat{v}(0)=0$. We can now use the updated value of $v$ to recompute $f$ and run the loop until the desired convergence is achieved (measured either by the change in $v$, or by computing the residual divergence of the stress field in the Fourier domain). The recursive application of the Green's function $\Gamma^{0}$ (which comes from $C^{0}$ ) can be shown to be simply the Neumann expansion (power series) of the actual Green's function $\Gamma$ (which comes from the nonuniform $C)$, and so the method converges to the full heterogeneous strain field. Note that this method may suffer from ringing (i.e. Gibb's phenomenon) near discontinuities if $C$ is highly discontinuous.

We use a more robust version of this FFT-based iterative solver, based on the Augmented Lagrangian (AL) method of Michel et al. [59] to solve the elastic problem (3.32). The modified algorithm conceptually consists of iteratively adjusting two strain and two stress fields. By design, one of the strain fields is compatible and one of the stress fields is in equilibrium, while the other strain and stress fields are related through the constitutive relation. The iterative procedure is conceived to make the pairs of strain and stress fields to converge to each other. At convergence, the method delivers a compatible strain field related with an equilibrated stress field through the local constitutive equation (see also [42] for details).

For loading step $n+1$, and given the state from the previous step $\varepsilon^{n},\left\{\lambda_{i}^{n}\right\},\left\{\gamma_{\alpha}^{n}\right\}$, we 
solve iteratively for $\varepsilon^{n+1}$ with imposed macroscopic (average) strain $\left\langle\varepsilon^{n+1}\right\rangle=\bar{\varepsilon}^{n+1}$ :

- Initialize for $k=0: \varepsilon^{k}=\varepsilon^{n}, s^{k}=C \cdot\left(\varepsilon^{n}-\varepsilon_{t}^{n}-\varepsilon_{p}^{n}\right)$

- Iterate:

1. $\tau^{k+1}=s^{k}-C_{0} \cdot \varepsilon^{k}$

2. $\hat{\tau}^{k+1}=\mathrm{FFT}\left(\tau^{k+1}\right)$

3. $\hat{e}^{k+1}=-\hat{\Gamma_{0}} \cdot \hat{\tau}^{k+1}, \hat{e}^{k+1}(0)=\bar{\varepsilon}^{n+1}$

4. $e^{k+1}=\operatorname{IFFT}\left(\hat{e}^{k+1}\right)$

5. $\varepsilon^{k+1}=\left(C+C_{0}\right)^{-1} \cdot\left(s^{k}+C_{0} \cdot e^{k+1}+C \cdot\left(\varepsilon_{t}^{n}+\varepsilon_{p}^{n}\right)\right)$

6. $s^{k+1}=s^{k}+C_{0} \cdot\left(e^{k+1}-\varepsilon^{k+1}\right)$

- Check for convergence:

$$
\frac{\left\|s^{k+1}-C \cdot\left(\varepsilon^{k+1}-\varepsilon_{t}^{n}-\varepsilon_{p}^{n}\right)\right\|_{\max }}{\left\|s^{k+1}\right\|_{\max }}<\text { tolerance, } \quad \frac{\left\|e^{k+1}-\varepsilon^{k+1}\right\|_{\max }}{\left\|\varepsilon^{n+1}\right\|}<\text { tolerance }
$$

- If converged, update $\varepsilon^{n+1}=\varepsilon^{k+1}$.

In the above, $\tau$ is the so-called polarization stress field, $e$ is the auxiliary (compatible) strain field, and $s$ is the auxiliary (equilibrated) stress field. $C_{0}$ is a solver parameter whose value affects the rate of convergence of the algorithm, but not the final solution, and $\Gamma_{0}$ the Green's operator associated with $C_{0}$ [89]. In the results that follow, the value of $C_{0}$ is 
chosen to be the arithmetic mean of all the local stiffness matrices; the numerical tolerance for convergence is $10 \times 10^{-6}$.

We then use the kinetic relations (3.33) and (3.34) to explicitly compute the new volume fractions $\left\{\lambda_{i}^{n+1}\right\}$ and slip activities $\left\{\gamma_{\alpha}^{n+1}\right\}$.

\subsection{Enforcement of constraints}

To enforce the constraints in Eq. (3.33), we first check non-negativity on each volume fraction individually, and correct if necessary. Given a newly computed $\lambda^{n+1}$,

$$
\lambda_{i}^{n+1}= \begin{cases}0, & \text { if } \lambda_{i}^{n+1}<0 \\ \lambda_{i}^{n+1}, & \text { otherwise }\end{cases}
$$

We then enforce the constraint on the total volume fraction,

$$
\lambda_{i}^{n+1}= \begin{cases}\lambda_{i}^{n+1}, & \text { if } \sum_{i=1}^{N} \lambda_{i}^{n+1} \leq 1 \\ \frac{\lambda_{i}^{n+1}}{\sum_{i=1}^{N} \lambda_{i}^{n+1}}, & \text { otherwise }\end{cases}
$$

which is equivalent to a radial return mapping back onto the face of the hyperplane which intercepts each axis at unity. An argument could be made for making a normal projection back onto the constraint surface, though for small increments, the two methods differ negligibly. 


\section{Chapter 4}

\section{Anti-plane Shear Results}

\subsection{Standard specimen}

We generate a polycrystal by picking 512 spatial points at random, and construct a periodic Voronoi tesselation using the DelaunayTri function in MATLAB ${ }^{\circledR}$. We then assign the orientation of each grain from a uniform random distribution of polar angles. We display most results for a specific realization, and this is shown in Figure 4.1(a). To illustrate the details of the fields, we often display the strain fields in one quarter of the domain that is also marked in the figure.

The energy density of material is given as

$$
W\left(\varepsilon,\left\{\lambda_{i}\right\},\left\{\gamma_{\alpha}\right\}\right)=W^{0}\left(R(x) \varepsilon,\left\{\lambda_{i}\right\},\left\{\gamma_{\alpha}\right\}\right)
$$

with

$$
\varepsilon_{i}^{t}(x)=R(x) \varepsilon_{i}^{t, 0}, \quad \varepsilon_{\alpha}^{p}(x)=R(x) \varepsilon_{\alpha}^{p, 0} .
$$




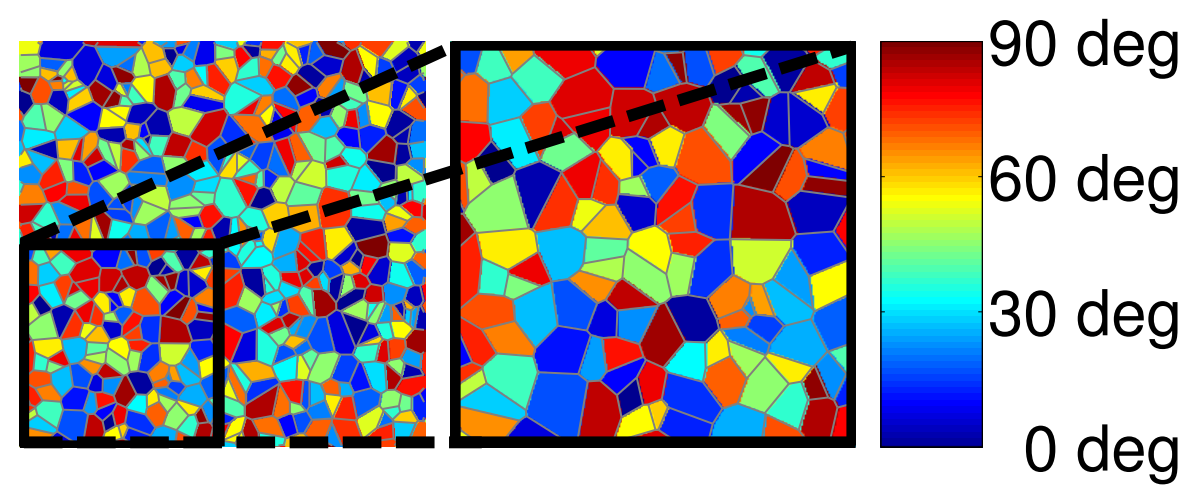

(a)

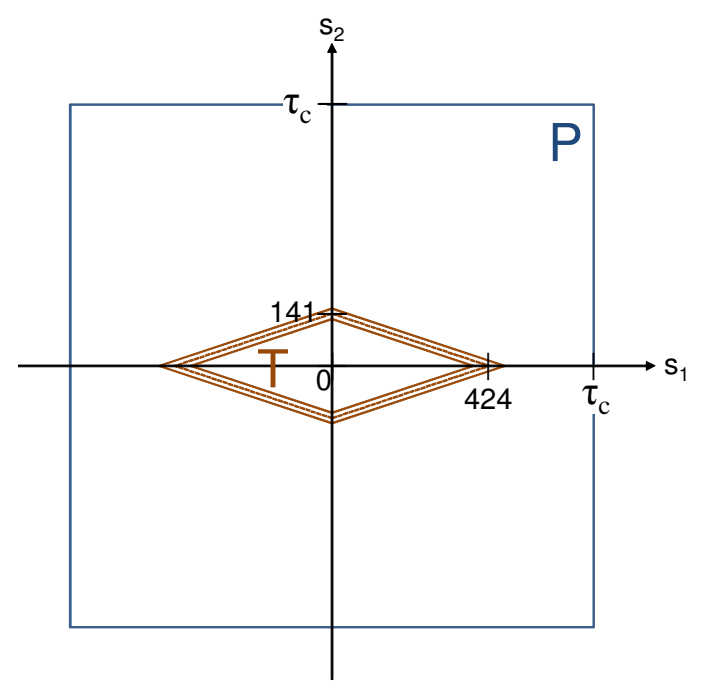

(b)

Figure 4.1: (a) The standard polycrystal with 512 grains, and a zoomed in region where we display strain fields in subsequent figures. (b) The transformation surface of the standard material, and an arbitrary plastic yield surface. The same transformation surface was used for all of the results presented herein, but $\tau_{c}$ was varied from $141 \mathrm{MPa}$ to $\infty$. There are three transformation surfaces, the outer (respectively middle and inner) corresponding to the forward (respectively thermodynamic and reverse) transformation. 
$R(x)$ is the rotation that maps the lattice of reference grain to that of the grain at $x, W^{0}$ is the energy density of the reference grain, $\varepsilon_{i}^{t, 0}$ is the transformation strain of the $i$ th variant of the reference grain and $\varepsilon_{\alpha}^{p, 0}$ is the strain of the $\alpha$ th slip system. We consider a reference material with four variants of martensite with transformation strains

$$
\varepsilon_{1}^{t, 0}=\left(\begin{array}{c}
\alpha \\
\beta
\end{array}\right), \quad \varepsilon_{2}^{t, 0}=\left(\begin{array}{c}
-\alpha \\
\beta
\end{array}\right), \quad \varepsilon_{3}^{t, 0}=\left(\begin{array}{c}
-\alpha \\
-\beta
\end{array}\right), \quad \varepsilon_{4}^{t, 0}=\left(\begin{array}{c}
\alpha \\
-\beta
\end{array}\right),
$$

and two slip systems, given by the plastic strain directions

$$
\varepsilon_{\alpha}^{p, 0}=\left(\begin{array}{c}
1 \\
0
\end{array}\right), \quad \varepsilon_{\alpha}^{p, 0}=\left(\begin{array}{c}
0 \\
1
\end{array}\right)
$$

The material parameters we choose are

$$
\begin{gathered}
C=\left(\begin{array}{cc}
70 & 0 \\
0 & 77
\end{array}\right) \text { GPa, } w=6 \times 10^{6} \mathrm{~J} / \mathrm{m}^{3}, \\
\alpha=0.0141, \quad \beta=0.0424, \\
d_{c}=0.6 \times 10^{6} \mathrm{~J}^{3} \mathrm{~m}^{3}, \quad h_{t}=1.984 \times 10^{-9} \mathrm{MPa}^{-1}, \\
\tau_{c}=424 \mathrm{MPa}, \quad h_{p}=7.14 \times 10^{-12} \mathrm{MPa}^{-1}
\end{gathered}
$$

unless we specify otherwise. Note that we have chosen the elastic modulus to be anisotropic, 
and so the polycrystal is not elastically homogeneous.

The set of recoverable strains for a single crystal as defined in [15] as all possible strains associated with the variants of martensite

$$
\mathcal{S}=\left\{\varepsilon:\left|\varepsilon_{1}\right| \leq 0.0141,\left|\varepsilon_{2}\right| \leq 0.0424\right\}
$$

is a rectangle with sides $0.04 \times 0.12$ centered at the origin. The Taylor inner bound on the set of recoverable uniaxial strains for an equiaxed polycrystal

$$
\mathcal{T}=\cap_{R} R \mathcal{S}=\{\varepsilon:|\varepsilon| \leq 0.0141\}
$$

is a ball of radius 0.0141 centered at the origin.

Since $w>0$, the material prefers austenite in its stress-free state. When subjected to a uniaxial stress, the single crystal begins to transform when $\min _{i} d_{i}^{\lambda}=d_{c}$ and this defines a transformation yield surface

$$
\mathcal{Y}^{t}=\left\{\sigma:|\sigma|=\min _{i} \frac{w+d_{c}}{\hat{\sigma} \cdot \varepsilon_{i}^{t}}\right\}
$$

This is the convex dual of the set of recoverable strains [16], and is shaped like a diamond in our case. This is shown in Figure 4.1(b) as the outer-most diamond. The inner-most diamond is the set obtained by replacing $-d_{c}$ with $+d_{c}$ in the formula above and represents the yield set for the reverse transformation. The central diamond is the equilibrium 
transformation surface we would have if $d_{c}$ were zero. The Sachs inner bound on the transformation yield surface for an equiaxed polycrystal

$$
\mathcal{Y}_{S}^{t}=\cap_{R} R \mathcal{Y}^{t}=\{\sigma: \sigma \leq 146 M P a\}
$$

is a ball of radius $146 \mathrm{MPa}$ centered at the origin.

The square in Figure 4.1(a) shows the plastic yield set

$$
\mathcal{Y}^{p}=\left\{\sigma:\left|\sigma_{1}\right| \leq \tau_{c},\left|\sigma_{2}\right| \leq \tau_{c}\right\}
$$

for $\tau_{c}=424 \mathrm{MPa}$.

All simulations are conducted at a simulated loading rate of $5 \times 10^{-5} \mathrm{~s}^{-1}$.

\subsection{Uniaxial loading}

\subsubsection{Superelasticity}

We begin by examining superelasticity with no plasticity by setting the yield strength to infinity. We load the specimen in strain control to $4 \%$ macroscopic strain in the $x$-direction, and then return in strain control to zero strain.

Figure 4.2 shows the macroscopic stress-strain curve as well as the strain field. As loading begins, the behavior is initially elastic. However, at a stress of around $142 \mathrm{MPa}$, we see the first evidence of transformation in a small region of an isolated grain (at a 


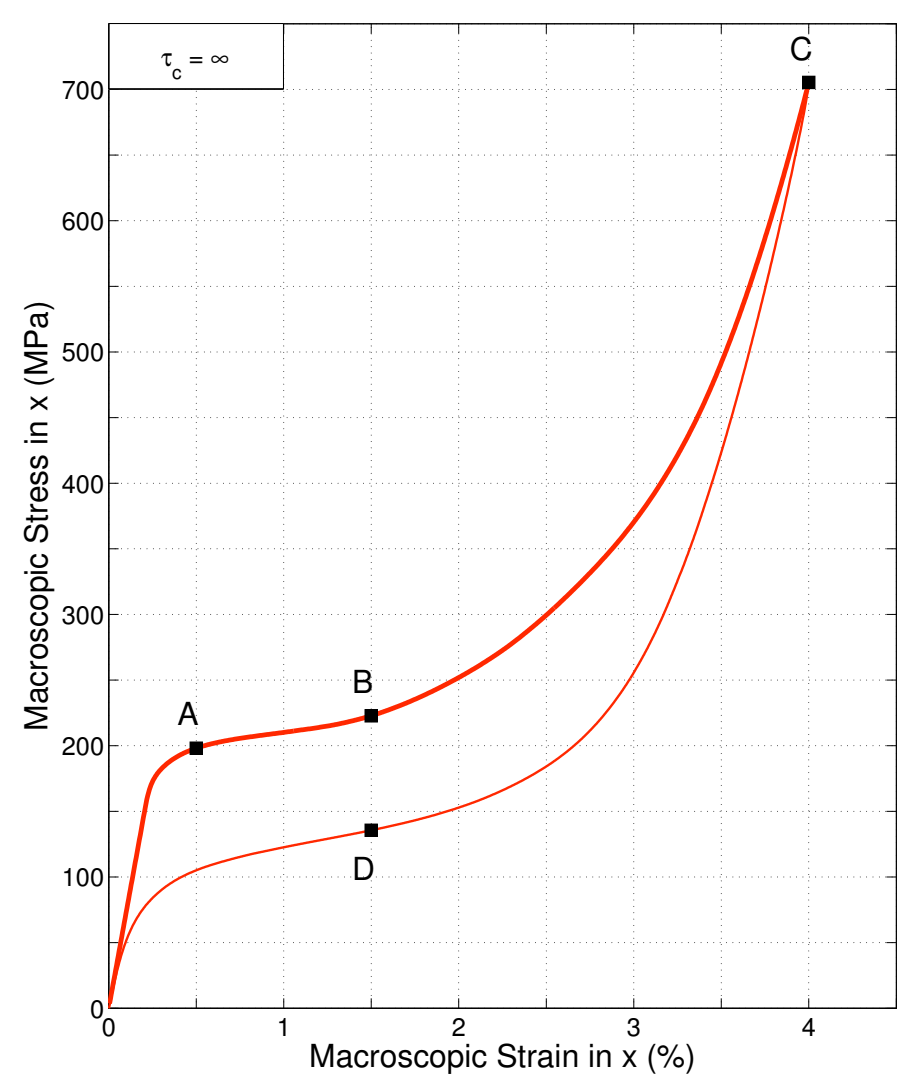

(a)

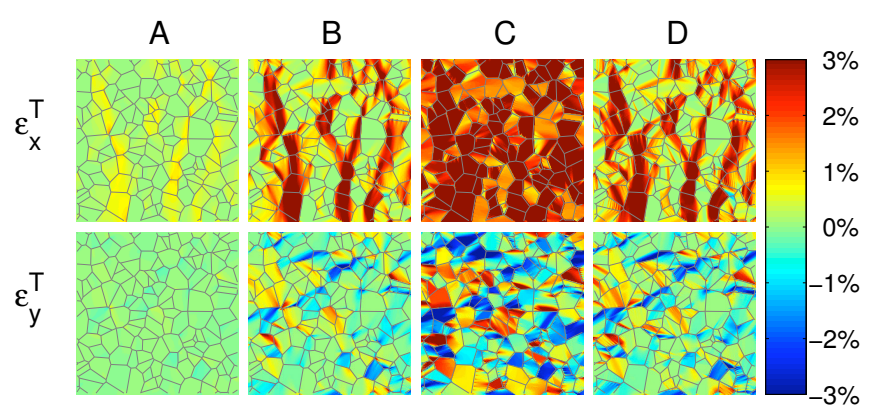

(b)

Figure 4.2: Superelasticity with no plasticity. (a) The overall stress-strain relation. (b) The transformation strain field at the macroscopic initiation of transformation, at the beginning of reorientation and at the macroscopic saturation of the transformation. 
single pixel of the calculation). Bhattacharya and Schlömerkemper [16] predicted that the onset of transformation would occur in an minute region of an isolated grain when the stress reaches the Sachs bound. However, the first appearance occurs at a value of stress that is less that the Sachs bound of $146 \mathrm{MPa}$. The reason for this is that the elastic modulus is heterogeneous (as opposed to the assumption in [16]) and therefore one has stress concentrations, especially at the triple junctions between grains. It also follows that the stress at which the first transformation occurs will depend on the numerical resolution. This is analogous to the observation of Brenner et al. [17] in the context of plasticity. However, the amount of transformation is very small and we do not see any consequence in the macroscopic behavior.

We see the macroscopic initiation of transformation at a stress of around $170 \mathrm{MPa}$, and the stress-strain curve displays an elbow. The strain field at the point marked A is shown in the figure. Note that the transformation occurs in patches and it is largely oriented with the load (i.e, the strain is largely in the imposed loading $x$-direction with little transverse strain).

Beyond this point, the transformation progresses and the strain increases rapidly. The strain field is shown for the point marked B in the figure. Note that the transformation is not uniform, but proceeds in localized bands perpendicular to the loading direction (consistent with the compatibility). Importantly, there are non-transformed grains and we see small patches of significant transverse strain. As the transformation is resisted by poorly oriented grains, a complex stress field develops and variants not necessarily aligned 
with the load begin to appear. In other words, the martensite begins to reorient.

The resistance to transformation, the amount of reorientation and hardening begins to increase beyond the point $\mathrm{B}$. So, by the time we reach the point $\mathrm{C}$, the transformation appears to be macroscopically saturated. However, the local strain field shows that there are regions that are untransformed or incompletely transformed.

All of these results - initiation in local regions, progress through localized bands and incomplete saturation - are consistent with the experimental observations of Brinson et al. [19] and Daly et al. [26].

We now begin to unload. The unloading is initially largely elastic, and thus the incremental modulus of unloading is larger than that of loading close to the point $\mathrm{C}$. The reverse transformation broadly follows the reverse sequence from that of transformation. In other words, the last region to transform to martensite is typically the first region to transform back to austenite and so forth. Indeed, comparing the strain fields at point D and point B which share the same macroscopic strain shows that the strains field are very similar, though not identical. Further, the reverse sequence means that reverse transformation begins in small isolated regions, then in increasing regions, and lastly, the final bits of martensite transform to austenite close to zero stress. For our choice of parameters, the entire specimen returned to austenite at zero load.

Further cycles repeat the sequence. 


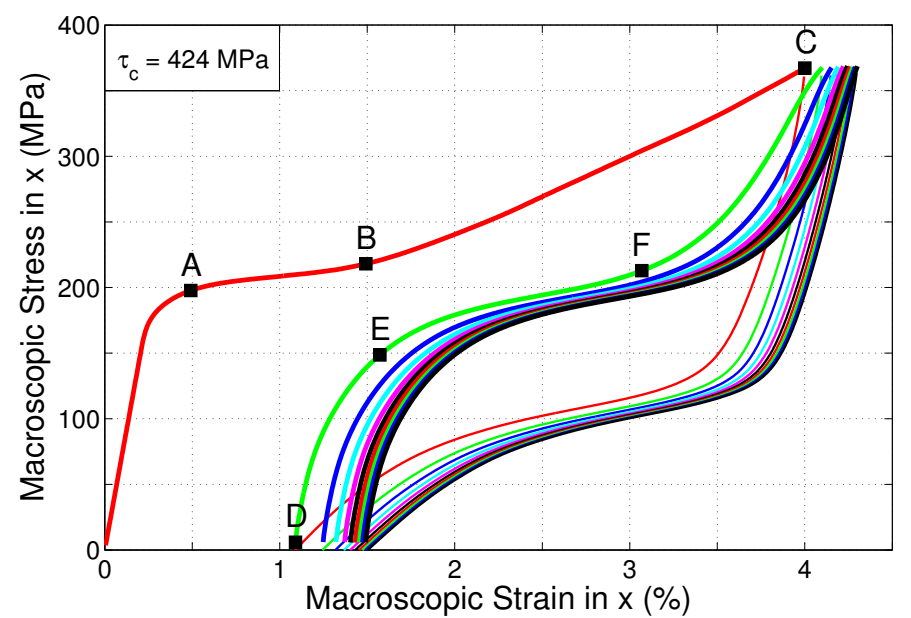

(a)
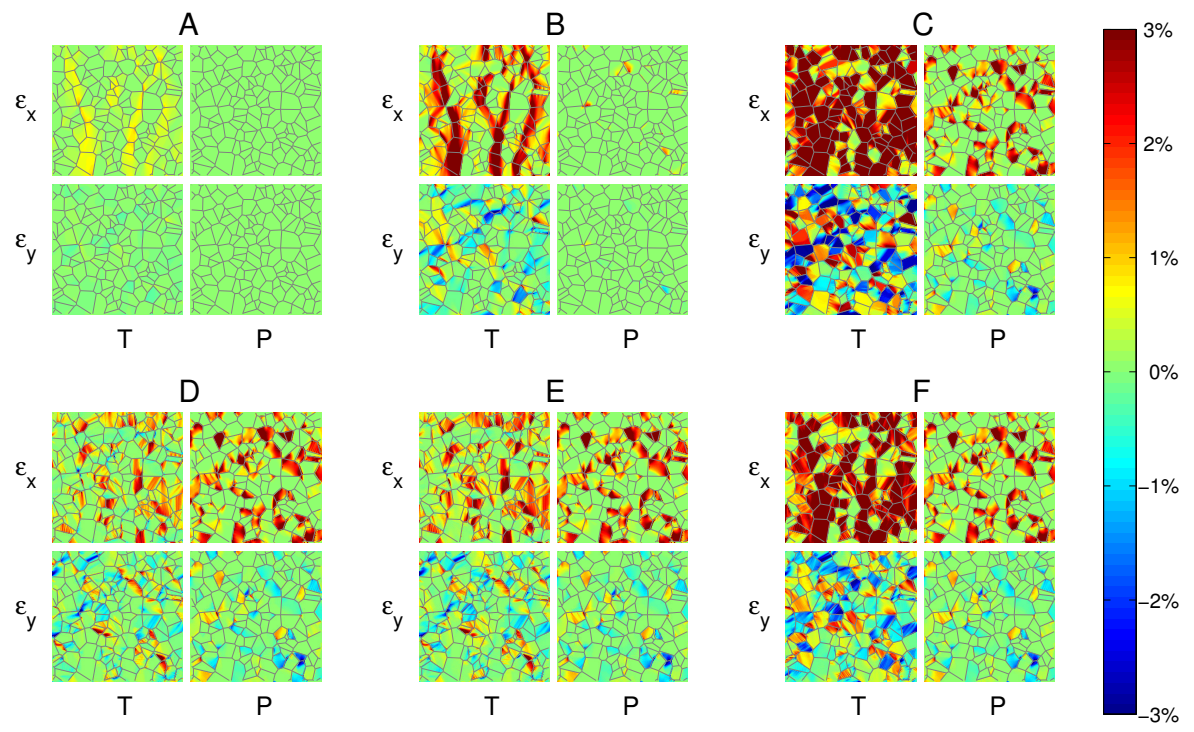

(b)

Figure 4.3: The interplay between transformation and plasticity under the cycling load protocol for a critical resolved stress of $424 \mathrm{MPa}$. (a) The stress-strain response of a protocol where the specimen is loaded in strain-control to $4 \%$ macroscopic strain, unloaded in strain-control to zero stress and then reloaded in strain-control cycles between zero stress and the value of the stress at the end of the first strain-control cycle. (b) The strain fields corresponding to six points. In each panel, the left column displays the transformation strain while the right column displays the plastic strain. 


\subsubsection{Interplay between transformation and plasticity}

We now look at the interplay between plasticity and phase-transformation for the standard specimen. We begin with a cyclic loading protocol which is as follows. First, the standard specimen is subjected to a strain controlled loading till $4 \%$ macroscopic strain in the $x$ direction (the $y$-direction is constrained to have zero strain). The peak macroscopic stress is recorded, and the polycrystal is unloaded in strain-control until the macroscopic stress returns to zero. Subsequently, the specimen is loaded in strain-control until the peak load from the first cycle is achieved, and then unloaded to zero stress.

Figure 4.3 shows results for a specimen with a critical resolved stress of $424 \mathrm{MPa}$. The transformation initiates as in the case of superelasticity with the strain field at the point marked A being similar with no plasticity. The progress of transformation is similar till we reach the point $\mathrm{B}$ where reorientation and hardening start to occur. At this point, we begin to see plastic deformation in isolated grains that are oriented poorly for transformation. In other words, plasticity provides a bridge across non-transforming grains. Further, note that we see plastic deformation in these grains though the macroscopic stress of about 220 $\mathrm{MPa}$ is significantly smaller than the critical resolved stress of $424 \mathrm{MPa}$ because of the significant heterogeneity and concentration in the stress field.

We observe hardening beyond B but note that the amount of hardening is smaller than in superelasticity. This is for two reasons. First, plasticity provides an additional deformation mechanism. Second, plasticity continues to provide a bridge across non-transforming 
grains thereby enabling additional transformation. By the time we reach the point marked C, we see significant plasticity. We also see transformation and plastic strain in a direction perpendicular to the macroscopic loading indicating the complex stress field.

As we unload the specimen, reverse transformation begins. However, the regions with significant amounts of plasticity resist the reverse transformation in the neighborhood. Consequently, there is a residual stress field, residual martensite and residual strain in the macroscopically unloaded specimen (point marked D).

The residual fields affect the transformation behavior on subsequent loading. One has a very small elastic region — if at all — as the initiation of transformation is earlier and much more spread out. Further, the transformation and plastic fields at the end of initiation (point marked E) are quite different from the corresponding quantities in the virgin specimen (point marked A). Further still, the plateau is lower and smaller, and the transformation appears to saturate with significant hardening.

Subsequent cycles cause further evolution with similar but with diminishing evolution. The material ultimately settles into a limit loop (within the resolution of the simulation) at about 60 cycles.

These results are consistent with the experimental observations of Morin et al. [60]. Figure 10 of that work also shows similar incomplete recovery and evolution with cycling, as well as the change of shape of the hysteresis loop.

An important feature that emerges from the cases we have studied is the significant complexity in the strain and stress field. We probe this further in Figure 4.4 by examining 
50
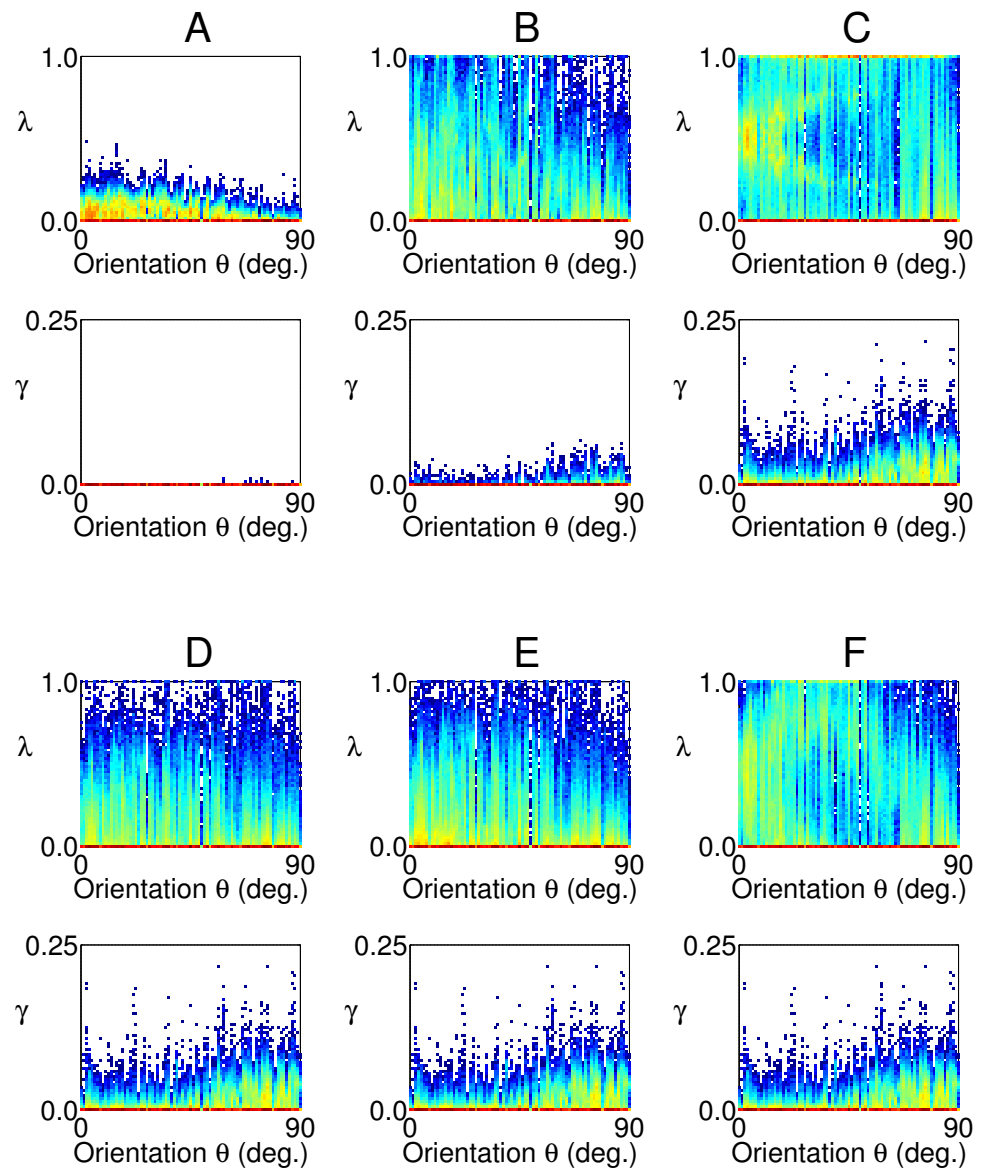

(a)
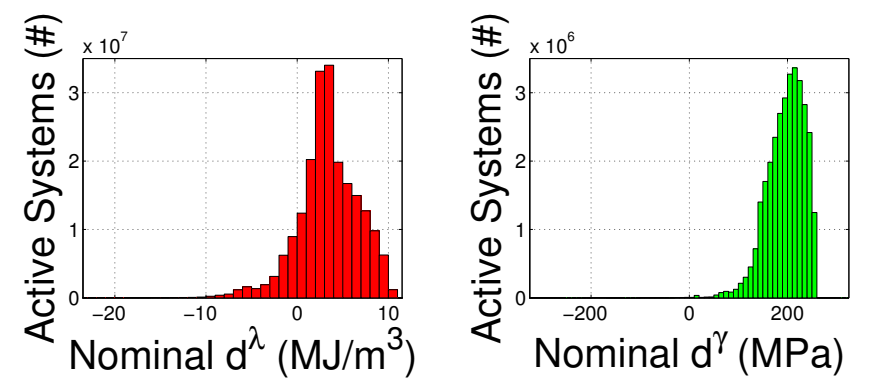

(b)

Figure 4.4: Statistical features of transformation and plasticity for the case shown in Figure 4.3. (a) Heat map showing the distribution of transformation volume fraction and plastic activity as a function of grain orientation. A white pixel represents no region at that orientation and volume fraction while blue to red pixels represent increasing regions with that orientation and volume fraction. (b) Cumulative histogram of transformation and plastic activity vs. the nominal driving force. 
some statistical measures. Figure 4.4(a) shows a heat map depicting the volume fraction of martensite (all variants) and plastic activity (all slip systems) vs. the local orientation at a computational pixel. We see a range of volume fractions and plastic activity at each orientation. Thus, the amount of transformation and amount of plasticity can be very different in grains that are oriented similarly, and in fact even in different parts of the same grain. We recall that a common assumption in the application of many mean-field theories, and in many experimental studies, is that grains of similar orientation deform similarly, and thus microstructural evolution can be predicted using average values of the micromechanical fields in the grains. Our results show that this assumption does not hold. This has also been recognized in other phenomena $[36,43,32]$ and led to the development of self-consistent models that take into account field fluctuations [36, 43]. This figure also shows the difference in state between the first and the second loading cycle (contrast A-B-C and E-F).

Another manifestation of how different the local stress can be from the global one is shown in Figure 4.4(b) which shows a cumulative histogram of the active systems vs. the nominal driving force (resolved stress) computed by projecting the macroscopic load on the local orientation. It counts the number of pixels that were active either in transformation or plasticity during the entire loading history (up to point $\mathrm{C}$ ) at each value of nominal driving force. It shows that transformation and plasticity occur at various nominal driving forces, including some where the nominal driving force is negative. Further, the peaks are unrelated to the critical values of $0.6 \mathrm{MJ} / \mathrm{m}^{3}$ in transformation and $424 \mathrm{MPa}$ in plasticity. 
The transformation peak is higher than the critical value since we use a rate-dependent model, while the plasticity is below the critical value due to the fluctuation in stress field as transformation proceeds heterogeneously across grains.

Figure 4.5 shows the analogous results for the situation where the critical resolved stress is reduced to $283 \mathrm{MPa}$. The results are similar except the plasticity sets in earlier (and again before the macrosopic stres reaches the critical resolved stress) and is more extensive.

We now examine the interplay between transformation and plasticity using a different loading protocol shown in Figure 4.6. The flow stress is $424 \mathrm{MPa}$. We load the specimen in strain control to $1 \%$, unload to zero stress and reload in strain control until an overall strain of $2 \%$, and repeat for total strains of $5 \%$ and $10 \%$. The first cycle where we load to a total strain of $1 \%$ is superelastic, and the specimen returns to the original austenite state with no plastic deformation on unloading. On the second cycle, the loading path coincides with that of the first cycle but we load to an overall strain of $2 \%$. As expected, we begin to see some hardening at around $1.5 \%$. As we unload, the reverse transformation is more diffuse compared to the the first cycle. The specimen appears to return to the original state with very little residual strain. However, there are small amounts of plasticity. This becomes evident on reloading where the transformation begins a little earlier and the transformation plateau is a little lower. The hardening is a little more so that it reaches approximately the earlier point at a total strain of $2 \%$. On the further loading, the material continues to harden, at a significant rate. On unloading after $5 \%$ total strain, the reverse transformation begins late and continues till zero load. There is a significant residual strain. On reloading, 


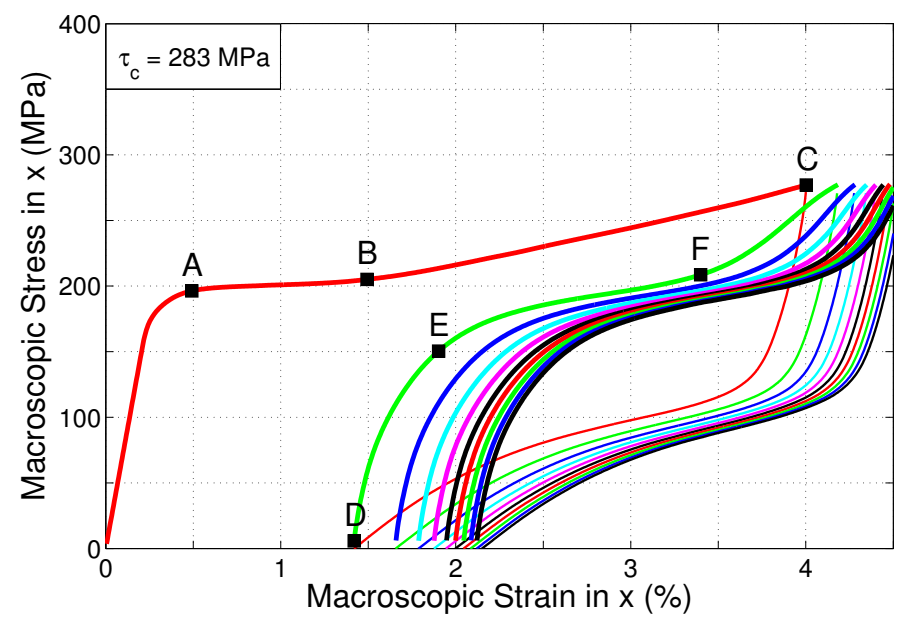

(a)
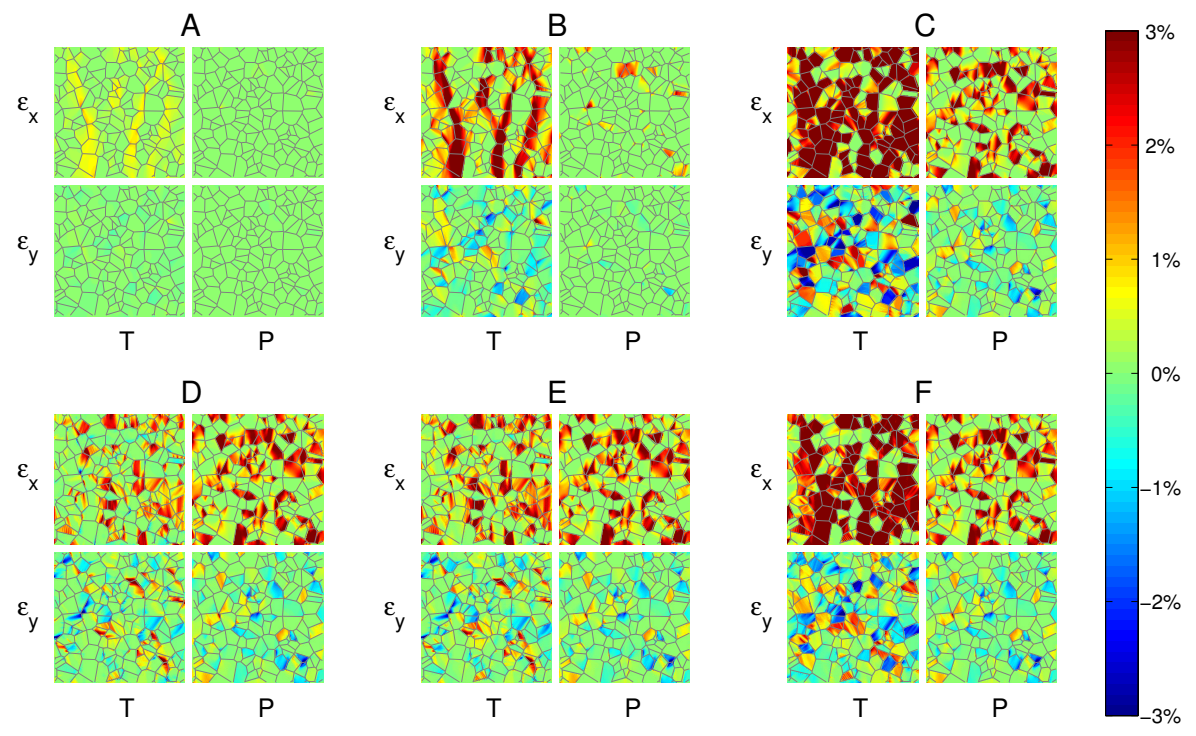

(b)

Figure 4.5: The interplay between transformation and plasticity under the cycling load protocol for a critical resolved stress of $283 \mathrm{MPa}$. (a) The stress-strain response of a loading protocol described in Figure 4.5. (b) The strain fields corresponding to six points. In each panel, the left column displays the transformation strain while the right column displays the plastic strain. 
54

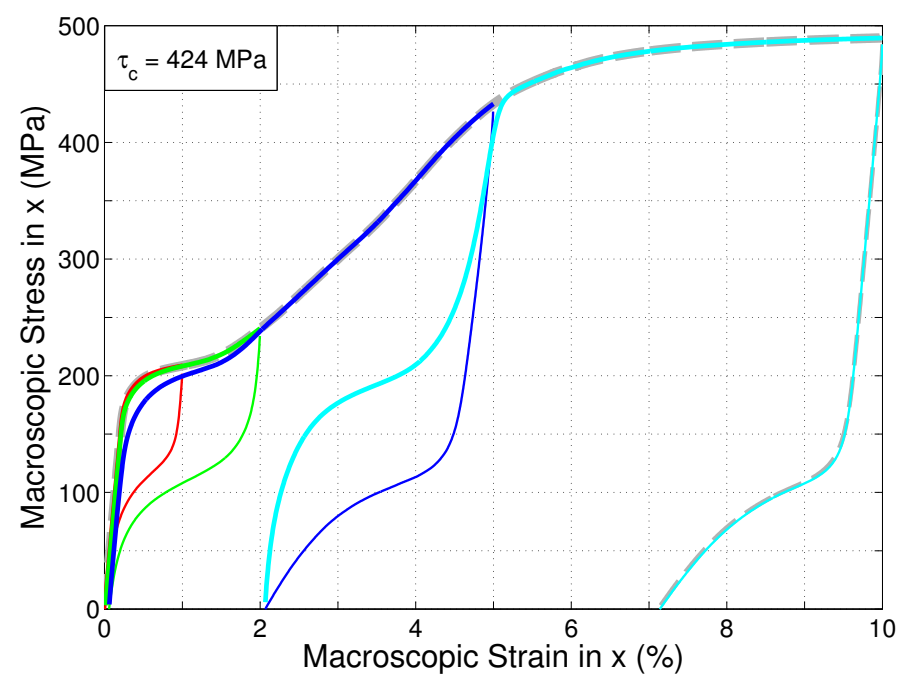

Figure 4.6: The interplay between transformation and plasticity under a increasing strain protocol for a critical resolved stress of $424 \mathrm{MPa}$. The specimen is loaded in strain control to $1 \%$, unloaded to zero stress and reloaded in strain control until an overall strain of $2 \%$, $5 \%$ and $10 \%$. The dashed line shows the response of a virgin material strained to $10 \%$.

the specimen transforms and hardens quickly to reach the previous level of stress at the previous maximum imposed strain, and then continues on. Eventually, the specimen yields plastically in a macroscopic manner. The unloading is then much quicker.

Figure 4.6 also shows the response of a virgin specimen loaded to $10 \%$ strain in an uninterrupted manner. Note that every time we unload and reload, the macroscopic response of the material returns to the previous maximum point, and then responds like a virgin specimen on continued loading. 

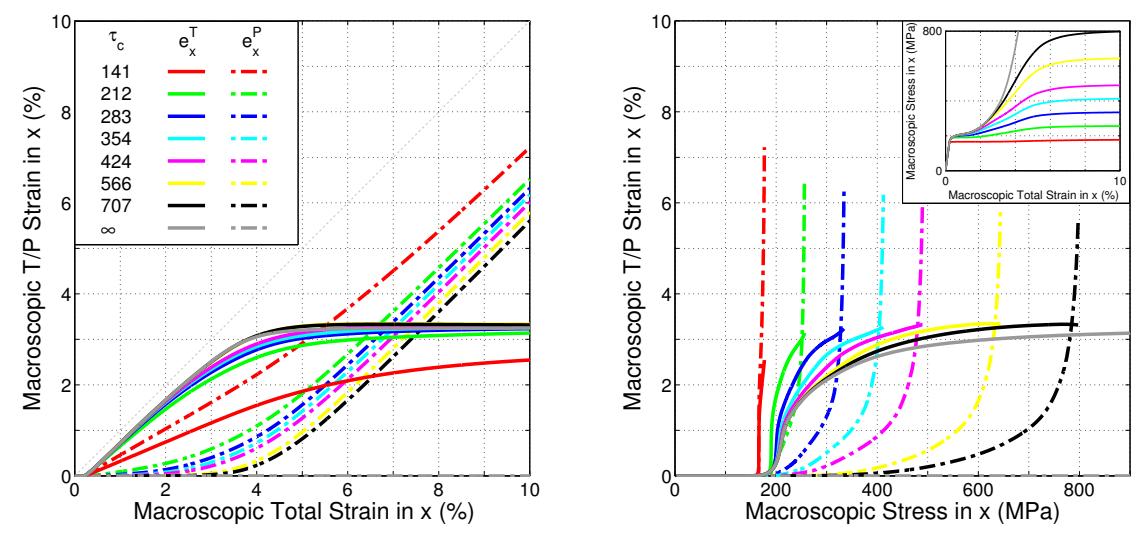

Figure 4.7: Decomposition of strain components for various yield strengths.

\subsubsection{The effect of flow stress}

The interplay between transformation and plasticity depends critically on the relative stress at which these occur. So we consider various flow stresses while keeping the transformation strains (and thus the critical stress for transformation) constant. The critical resolved stress ranges from $141 \mathrm{MPa}$ to $707 \mathrm{MPa}$ which traverses the range where the plastic yield surface for plasticity is completely within the transformation yield surface and vice-versa (see Figure 4.1).

We load the virgin standard specimen with a specified critical resolved stress to $10 \%$ strain in strain control, and monitor the total strain, the macroscopic transformation strain (i.e., the transformation averaged over all variants and all grains) and the macroscopic plastic strain (i.e., the plastic strain averaged over all slip systems and all grains). Figure 4.7 shows the macroscopic transformation (solid lines) and macroscopic plastic (dashed lines) strains as a function of the total strain. Aside from the case of flow stress of $141 \mathrm{MPa}$ where 


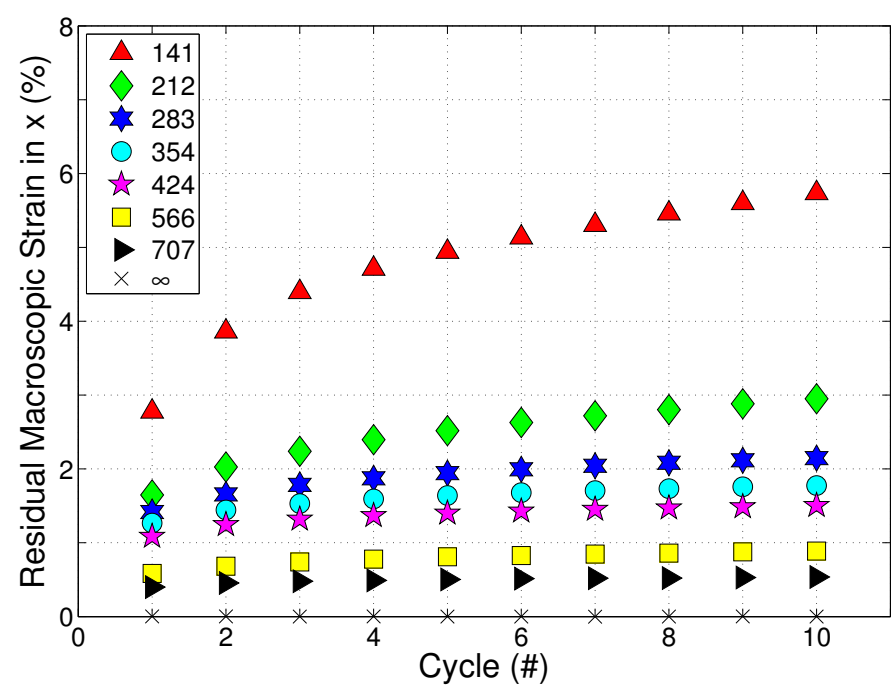

Figure 4.8: The evolution of the residual strain with cycling for various values of the critical resolved stress.

the plastic yield surface is essentially inside the transformation yield surface, the other cases follow a universal curve. In other words, the amount of transformation and the amount of of plastic strain is roughly independent of the critical resolved stress of plasticity. This is true despite the fact that the stress-strain curves look vastly different. This observation provides a key insight into the interplay between transformation and plasticity. As the material continues to transform, intergranular constraints resist the transformation leading to hardening (increased stress for incremental strain). The material will harden to the extent that is necessary to create plasticity to overcome the intergranular constraints and enable further transformation. In other words, the incompatibility of transformation is overcome by plasticity, and the stress reaches a level that is necessary for plasticity.

The effect of flow stress on the residual strain under cyclic loading is shown in Figure 


\section{7}

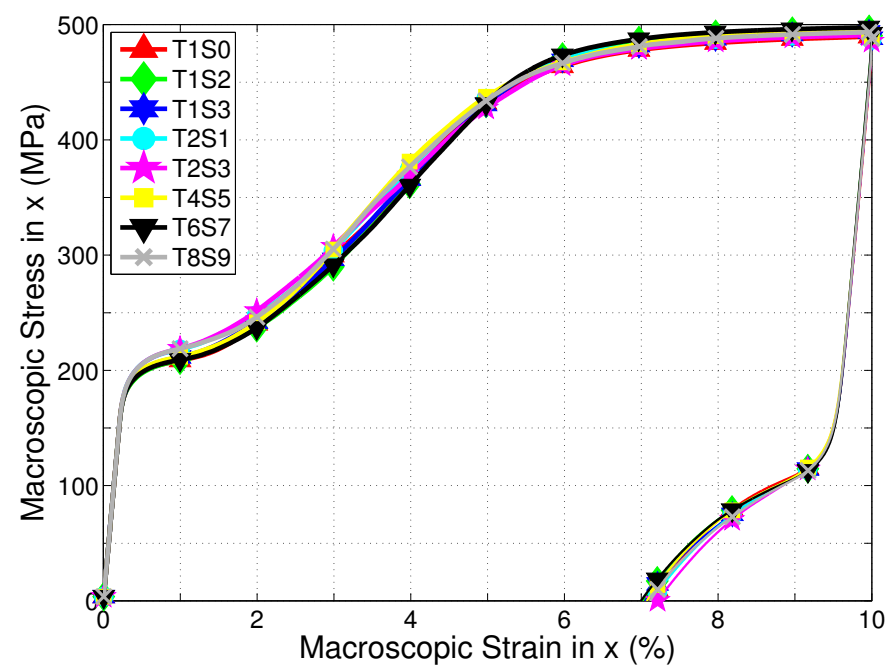

Figure 4.9: Specimen variation.

4.8. A virgin standard specimen with a specified critical resolved stress is loaded using the cyclic protocol described earlier (load till 4\%, unload and reload to the previous maximum stress). As expected, we see the residual strain increases with decreasing critical resolved stress, and to increase with cycling. We also see that the residual strain evolves significantly with cycling for low critical resolved stress, but settles down early for high critical resolved stress.

\subsubsection{Specimen variation}

We have so far displayed results with a standard specimen described in Section 4.1. Figure 4.9 shows the macroscopic stress-strain response of eight specimens. Each has 512 grains as before, and is generated by the same algorithm. We see that the results are very close to each other. Thus, we conclude that the results displayed earlier for the standard specimen 
are in fact representative results for an equiaxed specimen.

\subsubsection{Textured specimens}

As previously described, the spatial location and orientation of the 512 grains were assigned using a pseudo-random number generator. If the same seed value is used for picking both the spatial locations and the grain orientations, the orientations become perfectly correlated to the spatial locations, resulting in the banded microstructure shown in Figure 4.10(a). The macroscopic response of this textured microstructure is seen to differ substantially from the standard specimen with random texture. In particular, the transformation plateau is much more clearly defined, and the curvature of the following regime is opposite that of the standard specimen. The shape of this macroscopic stress-strain curve is more reminiscent of Nitinol wires and rods, which tend to be highly textured due to the manufacturing process.

\subsubsection{Loading rate}

We have noted that our model is rate sensitive, but thus far, we have interpreted the results from an essentially quasistatic perspective. To verify this interpretation, we vary

the uniaxial loading rates by factors of $\frac{1}{10}$ to 10 , relative to the standard loading rate of $50 \mu \mathrm{\epsilon} / \mathrm{s}$ (Figure 4.11). We see that there is no qualitative change in uniaxial loading response for rates up to twice the standard loading rate, and that the decomposition of the strain components again seems to follow a master curve, shown in Figure 4.12. 

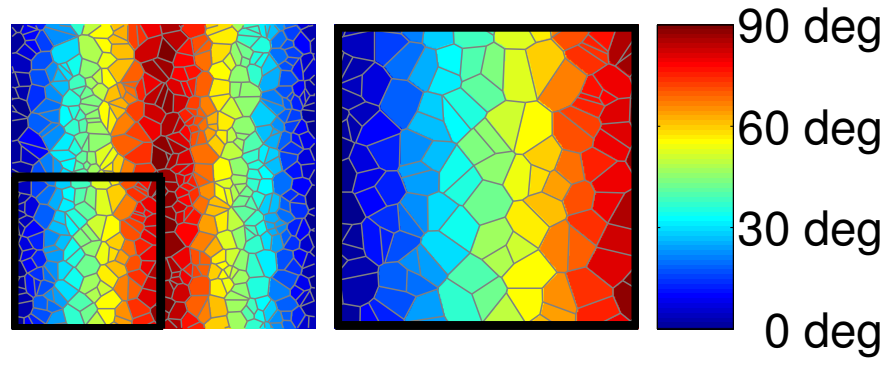

(a)

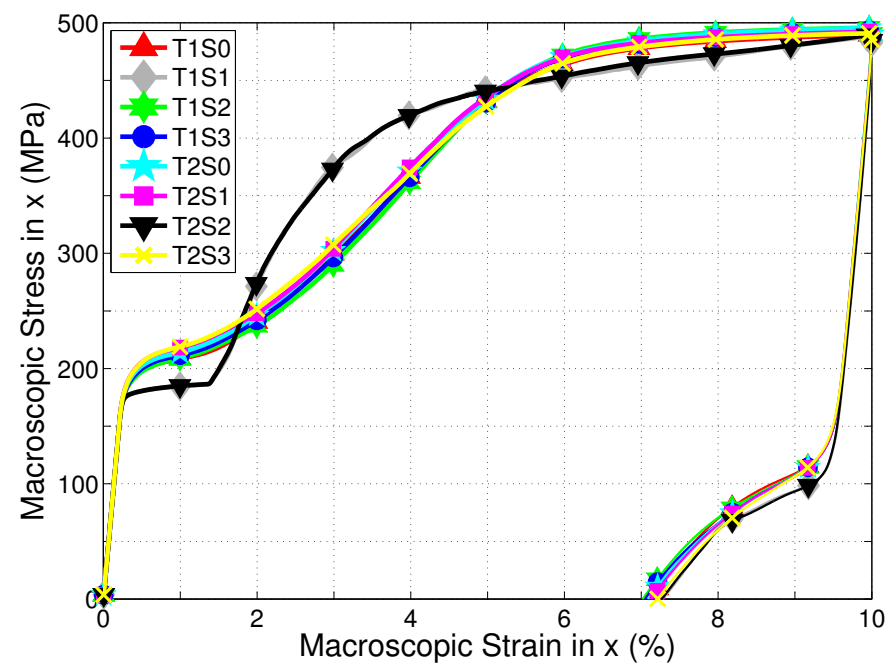

(b)

Figure 4.10: Effects of texture. (a) Gradient microstructure where the orientation and spatial location are highly correlated. (b) Comparison of macroscopic response of specimens with gradient microstructures (black and gray lines) to random microstructures. 


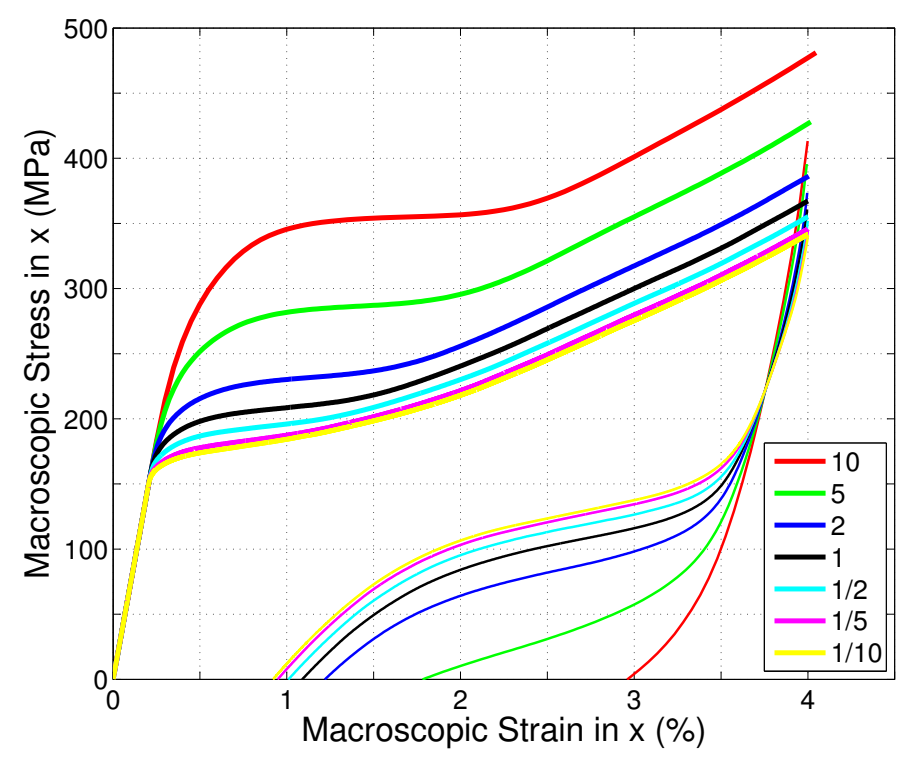

Figure 4.11: Uniaxial loading; reference loading rate (black) is $50 \mu \epsilon / \mathrm{s}$.
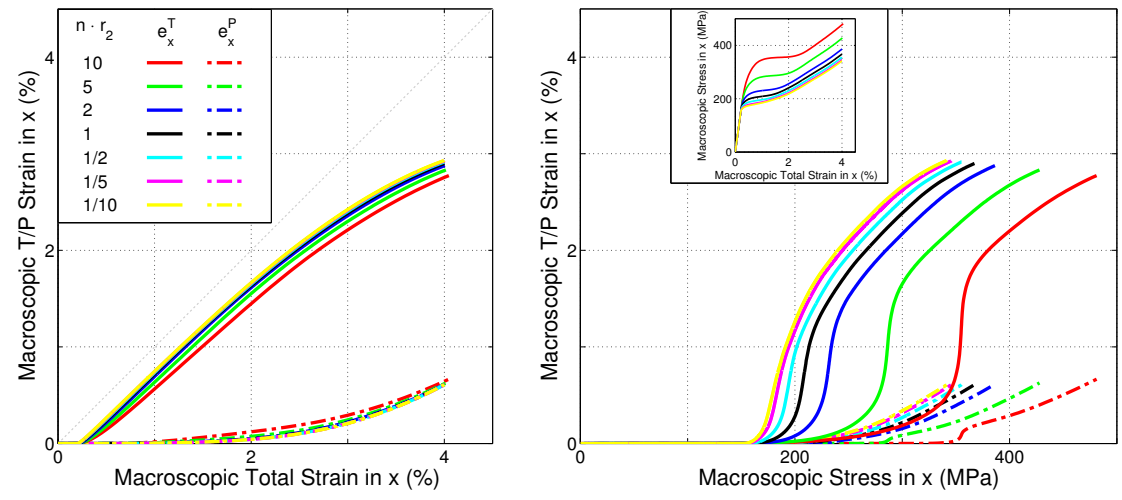

Figure 4.12: Decomposition of strain components for various loading rates. 

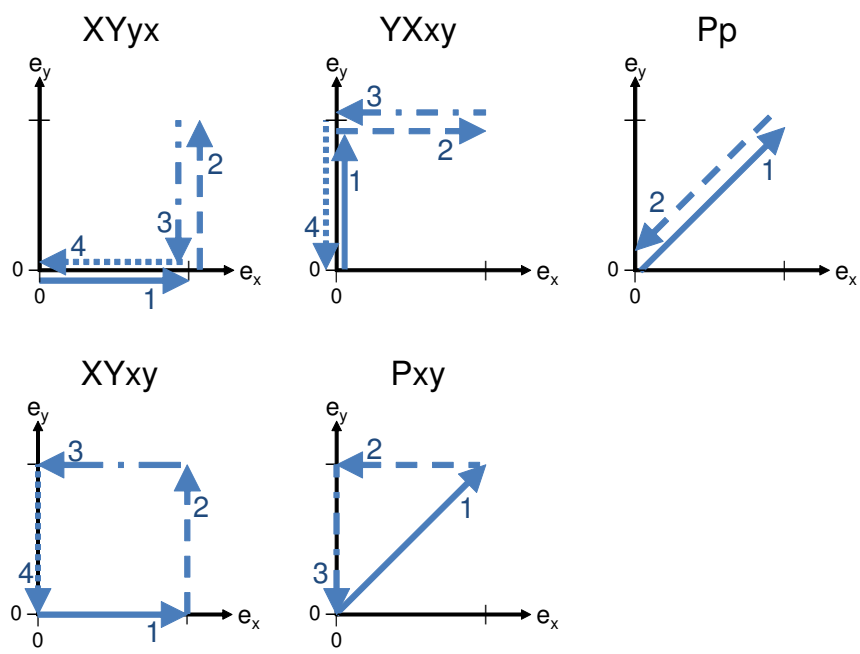

Figure 4.13: Five multiaxial straining protocols.

\subsection{Multiaxial and nonproportional straining}

We consider five multiaxial straining protocols all of which are in strain control shown schematically in Figure 4.13:

1. XYyx: Strain in $x$ till $\varepsilon_{x}$ reaches a specified value while holding $\varepsilon_{y}$ at zero, strain in $y$ till $\varepsilon_{y}$ reaches a specified value while holding $\varepsilon_{x}$ fixed, unstrain in $y$ till $\varepsilon_{y}$ reaches zero while holding $\varepsilon_{x}$ fixed and finally unstrain in $x$ till $\varepsilon_{x}$ reaches zero while holding $\varepsilon_{y}$ fixed.

2. YXxy: Strain in $y$ till $\varepsilon_{y}$ reaches a specified value while holding $\varepsilon_{x}=0$, strain in $x$ till $\varepsilon_{x}$ reaches a specified value while holding $\varepsilon_{y}$ fixed, unstrain in $x$ till $\varepsilon_{x}$ reaches zero while holding $\varepsilon_{y}$ fixed and finally unstrain in $y$ till $\varepsilon_{y}$ reaches zero while holding $\varepsilon_{y}$ fixed. 
3. Pp: Strain in both $x y$ with $\varepsilon_{x}$ and $\varepsilon_{y}$ equal till they reach a specified value, and then unstrain along the same path.

4. XYxy: Strain in $x$ till $\varepsilon_{x}$ reaches a specified value while holding $\varepsilon_{y}$ at zero, strain in $y$ till $\varepsilon_{y}$ reaches a specified value while holding $\varepsilon_{x}$ fixed, unstrain in $x$ till $\varepsilon_{x}$ reaches zero while holding $\varepsilon_{y}$ fixed and finally unstrain in $y$ till $\varepsilon_{y}$ reaches zero while holding $\varepsilon_{x}$ fixed.

5. Pxy: Strain in both $x y$ with $\varepsilon_{x}$ and $\varepsilon_{y}$ equal till they reach a specified value, unstrain in $y$ till $\varepsilon_{y}$ reaches zero while holding $\varepsilon_{x}$ fixed and finally unstrain in $x$ till $\varepsilon_{x}$ reaches zero while holding $\varepsilon_{y}$ fixed.

Figure 4.14 shows the stress response of the standard specimen with critical resolved stress of $424 \mathrm{MPa}$ subjected to the first three protocols. We begin by looking at the protocol XYyx which is indicated by the red curves. Figure 4.14(a) shows the response when the material is strained to a maximum of $1 \%$ in both directions. The response is completely superelastic. As we strain in the $x$ direction, the stress increases monotonically and uniaxially (consistent with the isotropy of the standard specimen). As we begin straining in the $y$ direction, the stress increases in the $y$ direction but the stress drops in the $x$-direction. This indicates that the martensite is reorienting to optimize the variants for the imposed strain, and reflects the shape of the so-called transformation yield surface. At the end of the loading stage, the $x$ and $y$ components of stress are not equal even though the corresponding strains are equal (to within the capability of the method). This shows the history 

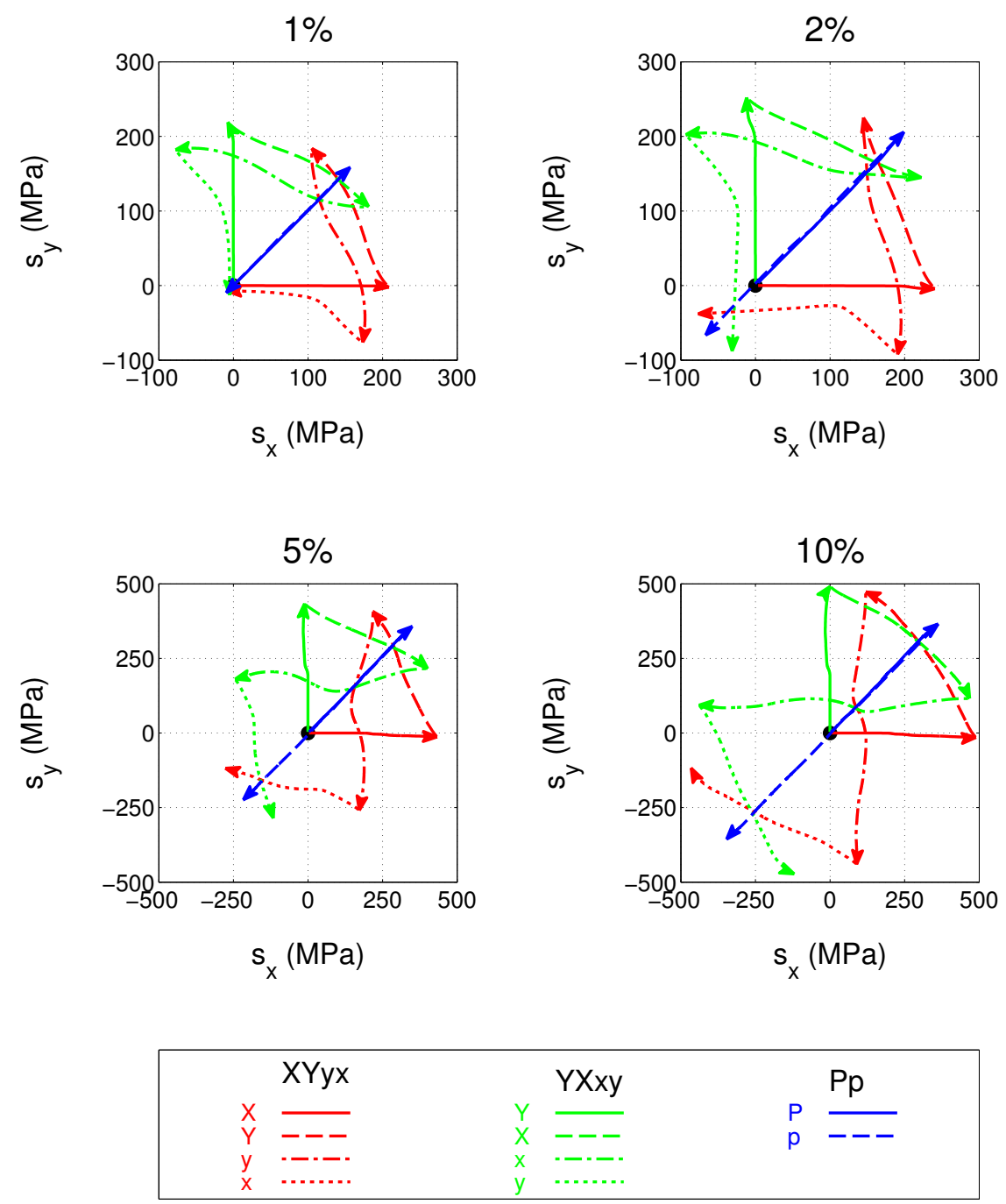

Figure 4.14: The evolution of the stress when the standard specimen with critical resolved stress of $424 \mathrm{MPa}$ is subjected to multiaxial straining according to protocols XYyx, YXxy and Pp till a total strain of $1 \%, 2 \%, 5 \%$ and $10 \%$. The red colored curves show protocol $\mathrm{XYyx}$, the green curves show the protocol YXxy and the blue diagonal lines show the protocol Pp. 
dependance of the material. As we reduce the strain in $y$, the stress does not follow the straining path and this is a manifestation of the hysteresis. All of this is consistent with the experimental observations of McNaney et al. [58] who studied the tension-torsion of thin-walled tubes. Further, the stress becomes zero and then negative in the $y$-direction even before the strain in the $y$-direction reaches zero. So we have to reverse load the specimen to return to zero strain. However, the stress macroscopically returns to zero as the strain returns to zero. In other words the material remains completely superelastic at this level of straining. Figure 4.14(b,c,d) shows the response when the material is strained to a maximum of $2 \%, 5 \%$ and $10 \%$ in both directions. The results are similar, but the specimen shows (increasing) plastic yielding, and so we are left with an (increasing) residual stress at zero strain.

The figure also reinforces the macroscopic symmetry of the standard specimen. Note that the stress associated with the proportional Pp straining and unstraining response always satisfies $\sigma_{x}=\sigma_{y}$. Further, the response to the XYyx protocol is symmetric to the YXxy protocol.

Figure 4.15 compares two protocols XYyx and YXyx where the straining segments are different, but the unstraining segments are the same. Not surprisingly the two protocols lead to the different stress states at the end of the straining. Consequently, the initial unstraining response is also different. However, in the case of pure transformation as in Figure $4.15(\mathrm{a})$ with $1 \%$ imposed strain as well as in the case of dominant plasticity as in Figure $4.15(\mathrm{~d})$, we see that the final portion of the unstraining response is similar. In other 

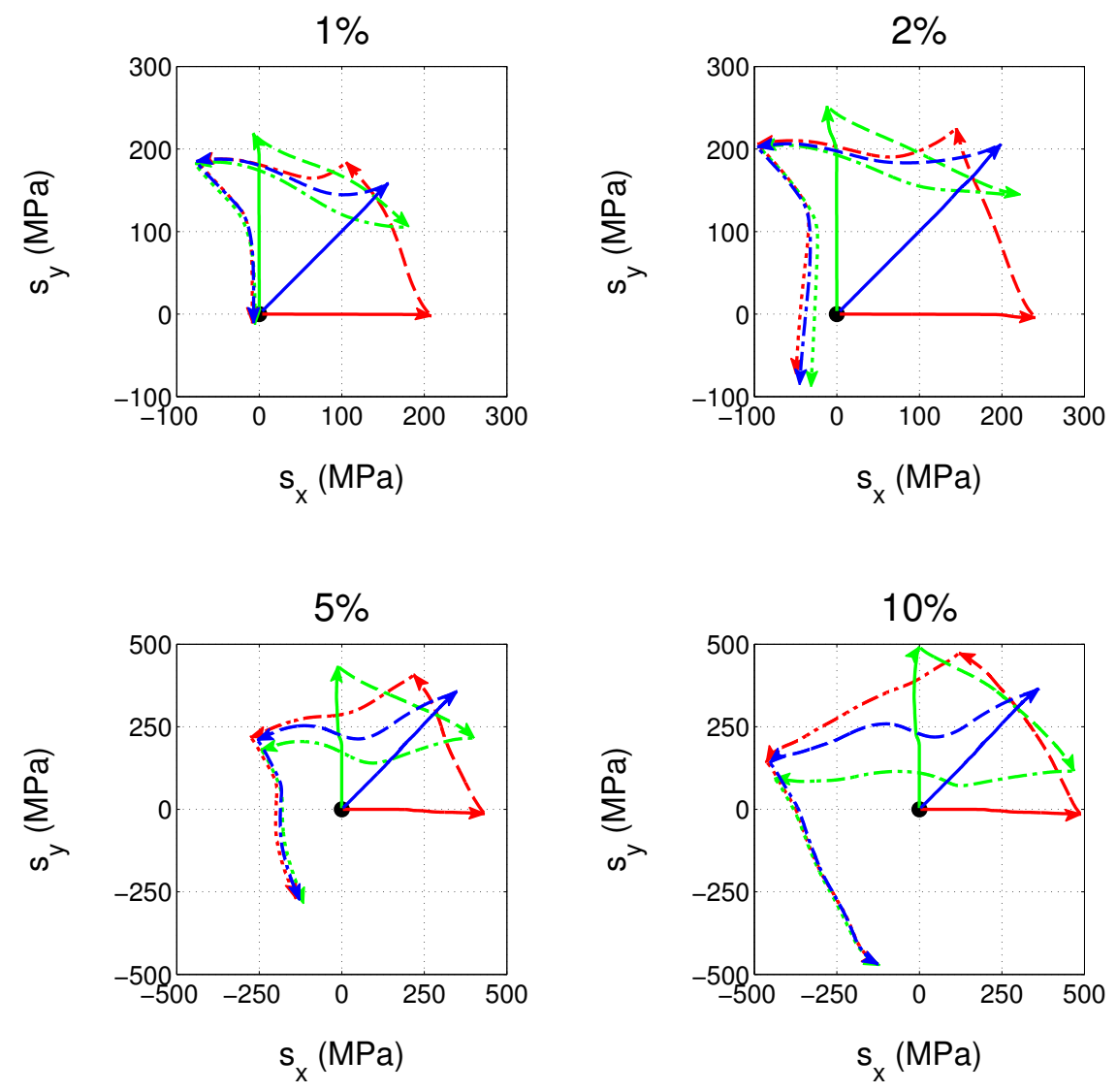

\begin{tabular}{|c|c|c|c|c|c|}
\hline & $X Y x y$ & & YXxy & & Pxy \\
\hline $\begin{array}{l}X \\
Y \\
Y\end{array}$ & $\overline{---}$ & $\begin{array}{l}Y \\
X\end{array}$ & $\overline{---}$ & $\begin{array}{l}P \\
x\end{array}$ & $\overline{---}$ \\
\hline $\begin{array}{l}x \\
y\end{array}$ & --- & $\begin{array}{l}x \\
y\end{array}$ & --- & $\hat{y}$ & - - . \\
\hline
\end{tabular}

Figure 4.15: The evolution of the stress when the standard specimen with critical resolved stress of $300 \mathrm{MPa}$ is subjected to multiaxial straining according to protocols YXxy and XYxy till a total strain of $1 \%, 2 \%, 5 \%$ and $10 \%$. The red colored curves show protocol XYxy, the green curves show the protocol YXxy and the blue curves show the protocol Pxy. 
words, when only one mechanism is active the specimen eventually forgets its (straining) history. However, when both mechanisms are active as in Figure 4.15(c,d), the final portion of the response and the end states are different. 


\section{Chapter 5}

\section{Generalized Framework}

\subsection{Linearized kinematics in 3-D}

We look towards generalized 3-D loading. We note that the previous anti-plane setting was unique in that the and that the additive decomposition of the strain was exact. We now introduce the approximation of 3-D linearized kinematics,

$$
\varepsilon=\frac{1}{2}\left(\nabla \mathbf{u}+(\nabla \mathbf{u})^{T}\right)
$$

which drops the nonlinear term $(\nabla \mathbf{u})^{T} \nabla \mathbf{u}$ from the Green-Lagrange strain. Due to linearity, we may again suppose an additive decomposition,

$$
\varepsilon=\varepsilon_{e}+\varepsilon_{t}+\varepsilon_{p}
$$


whose components may again be further decomposed,

$$
\begin{aligned}
& \varepsilon_{t}=\sum_{i=1}^{N} \lambda_{i} \varepsilon_{i}^{t}, \quad \varepsilon_{i}^{t}=\mathbf{U}^{i}-\mathbf{I}, \\
& \lambda_{i} \in[0,1], \quad \sum_{i=1}^{N} \lambda_{i} \leq 1, \quad \lambda_{0}=1-\sum_{i=1}^{N} \lambda_{i},
\end{aligned}
$$

and

$$
\begin{aligned}
& \varepsilon_{p}=\sum_{\alpha=1}^{M} \gamma_{\alpha} \varepsilon_{\alpha}^{p}, \quad \varepsilon_{\alpha}^{p}=\frac{1}{2}\left(\mathbf{s}_{\alpha} \otimes \mathbf{m}_{\alpha}+\mathbf{m}_{\alpha} \otimes \mathbf{s}_{\alpha}\right), \\
& \dot{\gamma}_{\alpha} \geq 0 .
\end{aligned}
$$

\subsection{Governing equations}

The formulation for the 3-D setting is similar to Sections 3.2 and 3.3, except we add the inertia,

$$
\begin{aligned}
\nabla \cdot \sigma+\mathbf{b} & =\rho \ddot{\mathbf{u}} \\
\dot{\epsilon} & =\sigma \cdot \dot{\varepsilon}-\nabla \cdot q+r \\
\dot{\mathcal{D}} & :=\sigma \cdot \dot{\varepsilon}-\dot{\epsilon}+T \dot{\eta}-\frac{\nabla T}{T} \cdot q \geq 0
\end{aligned}
$$

where $\rho$ is the mass density per unit volume and $\ddot{\mathbf{u}}$ is the second derivative of the displacement with respect to time (acceleration). 
We now postulate an additive form for the Helmholtz free energy $A=\epsilon-T \eta$, s.t.,

$$
A(\varepsilon, \lambda, \gamma, T)=W^{e}\left(\varepsilon^{e}, T\right)+W^{T}(T)+W^{t}(\lambda, T)+W^{p}(\gamma, T)+\ldots
$$

where $W^{e}$ is the elastic energy density, $W^{T}$ is the thermal energy density, $W^{t}$ represents the energy penalty associated with phase transformation, and $W^{p}$ represents the stored energy due to plastic work (essentially, plastic hardening); additional hardening potentials, including those with mixed dependence on transformation and hardening, could be added as desired without altering the proposed framework. Substituting $\epsilon=A-T \eta$ into (5.9), we get,

$$
\dot{\mathcal{D}}=\sigma \cdot \dot{\varepsilon}^{e}-\frac{\partial A}{\partial \varepsilon} \cdot \dot{\varepsilon}^{e}-\sum_{i=0}^{N} \frac{\partial A}{\partial \lambda_{i}} \dot{\lambda}_{i}-\sum_{\alpha=1}^{M} \frac{\partial A}{\partial \gamma_{\alpha}} \dot{\gamma}_{\alpha}-\frac{\partial W}{\partial T} \dot{T}-\eta \dot{T}-\frac{\nabla \theta}{\theta} \cdot \dot{q}
$$

We again define the stress, entropy, and the driving forces following Coleman and Noll [23] as

$$
\begin{aligned}
\sigma & =\frac{\partial A}{\partial \varepsilon^{e}}, & \eta & =\frac{\partial A}{\partial T} \\
d_{\lambda_{i}} & =-\frac{\partial A}{\partial \lambda_{i}}, & d_{\gamma_{\alpha}} & =-\frac{\partial A}{\partial \gamma_{\alpha}}
\end{aligned}
$$

and prescribe kinetic relations governing the evolution of the transformation and plasticity, 
assuming gradient flow [73],

$$
\dot{\lambda_{i}}=K_{t}\left(d_{\lambda_{i}}, T\right), \quad \dot{\gamma_{\alpha}}=K_{p}\left(d_{\gamma_{\alpha}}, T\right)
$$

such that the rate of change of each kinetic variable is some function of its associated driving force. Alternately, we introduce dissipation functions $D_{t}$ and $D_{p}$, consistent with the evolution laws, s.t.

$$
d_{\lambda_{i}}=\frac{\partial D_{t}\left(\dot{\lambda_{i}}, T\right)}{\partial \dot{\lambda_{i}}}, \quad d_{\gamma_{\alpha}}=\frac{\partial D_{p}\left(\dot{\gamma_{\alpha}}, T\right)}{\partial \dot{\gamma}_{\alpha}}
$$

where $D_{t}$ and $D_{p}$ are the inverse or "dual" potentials to $K_{t}$ and $K_{p}$, in that the "derivatives" of $D_{t}$ and $D_{p}$ are effectively $K_{t}^{-1}$ and $K_{p}^{-1}$. Since the inverse of the kinetic functions may be undefined, we must clarify that the derivatives in Eq. 5.14 are understood more generally as subdifferentials in the context of convex anaylsis (cf. [77]). As such, $\partial_{d_{\dot{\lambda}_{i}}} D_{t}\left(\dot{\lambda}_{i}, T\right)$ and $\partial_{\dot{d}_{\alpha}} D_{p}\left(\dot{\gamma}_{\alpha}, T\right)$ are chosen from a class of functions to be multi-valued at $\dot{\lambda}_{i}=0$ and $\dot{\gamma}_{\alpha}=0$, respectively, giving rise to stick-slip behavior (cf. $[3,1])$. 
Returning to Equation (5.8) and noting $\epsilon=A+T \eta$,

$$
\begin{aligned}
\dot{\epsilon} & =\dot{A}+\dot{T} \eta+T \dot{\eta} \\
& =\left(\frac{\partial A}{\partial \varepsilon} \dot{\varepsilon}+\sum_{i=1}^{N} \frac{\partial A}{\partial \lambda_{i}} \dot{\lambda}_{i}+\sum_{\alpha=1}^{M} \frac{\partial A}{\partial \gamma_{\alpha}} \dot{\lambda}_{\alpha}+\frac{\partial A}{\partial T} \dot{T}\right)+\dot{T} \eta+T \dot{\eta} \\
& =\left(\sigma \dot{\varepsilon}-\sum_{i=1}^{N} d_{i}^{\lambda} \dot{\lambda}_{i}-\sum_{\alpha=1}^{M} d_{\alpha}^{\gamma} \dot{\lambda}_{\alpha}-\eta \dot{T}\right)+\dot{T} \eta+T \dot{\eta} \\
& =\sigma \dot{\varepsilon}-\sum_{i=1}^{N} d_{i}^{\lambda} \dot{\lambda}_{i}-\sum_{\alpha=1}^{M} d_{\alpha}^{\gamma} \dot{\lambda}_{\alpha}+T \dot{\eta} .
\end{aligned}
$$

Equating (5.8) and (5.15), and assuming an adiabatic process $(q=r=0)$, we see that,

$$
T \dot{\eta}=-\sum_{i=1}^{N} d_{i}^{\lambda} \dot{\lambda}_{i}-\sum_{\alpha=1}^{M} d_{\alpha}^{\gamma} \dot{\lambda}_{\alpha}
$$

From the definition of the entropy (5.12), we get,

$$
\begin{aligned}
-\dot{\eta} & =\frac{\partial}{\partial t}\left(\frac{\partial A}{\partial T}\right) \\
& =\frac{\partial^{2} A}{\partial \varepsilon \partial T} \dot{\varepsilon}+\sum_{i=1}^{N} \frac{\partial^{2} A}{\partial \lambda_{i} \partial T} \dot{\lambda}_{i}+\sum_{\alpha=1}^{M} \frac{\partial^{2} A}{\partial \gamma_{\alpha} \partial T} \dot{\gamma}_{\alpha}+\frac{\partial^{2} A}{\partial T^{2}} \dot{T}
\end{aligned}
$$

We note that, in Equation (5.17), the first term on the right-hand side is associated with thermal expansion, the second term gives rise to the latent heat of transformation, the third term is the entropic change stored in the lattice due to plasticity, and the fourth term 
gives rise to the heat capacity. Substitution of (5.17) into (5.16) gives

$$
T\left(\frac{\partial^{2} A}{\partial \varepsilon \partial T} \dot{\varepsilon}+\sum_{i=1}^{N} \frac{\partial^{2} A}{\partial \lambda_{i} \partial T} \dot{\lambda}_{i}+\sum_{\alpha=1}^{M} \frac{\partial^{2} A}{\partial \gamma_{\alpha} \partial T} \dot{\gamma}_{\alpha}+\frac{\partial^{2} A}{\partial T^{2}} \dot{T}\right)=\sum_{i=1}^{N} d_{i}^{\lambda} \dot{\lambda}_{i}+\sum_{\alpha=1}^{M} d_{\alpha}^{\gamma} \dot{\gamma}_{\alpha}
$$

We define

$$
\begin{aligned}
\rho C_{v} & =T\left(\frac{\partial^{2} A}{\partial T^{2}}\right) \\
\beta_{\alpha} & =\frac{d_{\alpha}^{\gamma}-T\left(\frac{\partial^{2} A}{\partial \gamma_{\alpha} \partial T}\right)}{d_{\alpha}^{\gamma}}=\beta \\
\frac{L_{i}}{T_{c_{i}}} & =T\left(\frac{\partial^{2} A}{\partial \lambda_{i} \partial T}\right)=\frac{L}{T_{c}}
\end{aligned}
$$

where $C_{v}$ is interpreted as the heat capacity, $\beta$ is the so-called Taylor-Quinney coefficient, and $\frac{L_{i}}{T_{c_{i}}}$ is the latent heat associated with transformation. Substituting these parameters into (5.18), and ignoring the contributions due to thermal expansion and direct dissipation due to phase transition, we get,

$$
\rho C_{v} \dot{T}=\sum_{i=1}^{N} \frac{L}{T_{c}} \dot{\lambda}_{i}+\sum_{\alpha=1}^{M} \beta d_{\alpha}^{\gamma} \dot{\gamma}_{\alpha}
$$

In summary, Equation (5.22), along with (5.7), (5.12), and (5.14), gives rise to the 
systems of equations,

$$
\begin{aligned}
\nabla \cdot \frac{\partial A}{\partial \varepsilon}(\varepsilon, \lambda, \gamma, T) & =\rho \ddot{\mathbf{u}} \\
-\frac{\partial A}{\partial \lambda_{i}} & =\frac{\partial D_{t}\left(\dot{\lambda}_{i}, T\right)}{\partial \dot{\lambda}_{i}} \\
-\frac{\partial A}{\partial \gamma_{\alpha}} & =\frac{\partial D_{p}\left(\dot{\gamma}_{\alpha}, T\right)}{\partial \dot{\gamma}_{\alpha}} \\
\rho C_{v} \dot{T} & =\sum_{i=1}^{N} \frac{L}{T_{c}} \dot{\lambda}_{i}+\sum_{\alpha=1}^{M} \beta d_{\alpha}^{\gamma} \dot{\gamma}_{\alpha}
\end{aligned}
$$

which we must solve simultaneously subject to the constraints

$$
\begin{array}{r}
\lambda_{i} \in[0,1], \quad \sum_{i=1}^{N} \lambda_{i} \leq 1, \\
d_{i}^{\lambda} \dot{\lambda}_{i} \geq 0, \quad d_{\alpha}^{\gamma} \dot{\gamma}_{\alpha} \geq 0 .
\end{array}
$$

\subsection{Implicit time discretization and variational principle}

We note that the implicit time discretization of Equations (5.23)-(5.25) is given by,

$$
\begin{aligned}
\nabla \cdot \frac{\partial A}{\partial \varepsilon}\left(\varepsilon^{n+1}, \lambda^{n+1} \gamma^{n+1}, T^{n}\right) & =\rho \frac{\mathrm{u}^{n+1}-2 \mathrm{u}^{n}+\mathrm{u}^{n-1}}{(\Delta t)^{2}} \\
-\frac{\partial A}{\partial \lambda_{i}}\left(\varepsilon^{n+1}, \lambda^{n+1}, \gamma^{n+1}, T^{n}\right) & =\frac{\partial D_{t}}{\partial \dot{\lambda}_{i}}\left(\frac{\lambda_{i}^{n+1}-\lambda_{i}^{n}}{\Delta t}, T^{n}\right), \\
-\frac{\partial A}{\partial \gamma_{\alpha}}\left(\varepsilon^{n+1}, \lambda^{n+1}, \gamma^{n+1}, T^{n}\right) & =\frac{\partial D_{p}}{\partial \dot{\gamma}_{\alpha}}\left(\frac{\gamma_{\alpha}^{n+1}-\gamma_{\alpha}^{n}}{\Delta t}, T^{n}\right)
\end{aligned}
$$


where we have staggered the time step of the temperature. We note that, given $\varepsilon^{n+1}$, we can solve for $\lambda^{n+1}$ and $\gamma^{n+1}$ using (5.30) \& (5.31). We do so variationally (see, e.g., [66]). We define a pseudo-potential $g$, s.t.

$$
\begin{aligned}
& g\left(\lambda_{n+1}, \gamma_{n+1}\right)= \\
& \quad A\left(\varepsilon_{n+1}, \lambda_{n+1}, \gamma_{n+1}, T_{n}\right)-A_{n}+\Delta t D_{t}\left(\frac{\lambda_{n+1}-\lambda_{n+1}, T_{n}}{\Delta t}\right)+\Delta t D_{p}\left(\frac{\gamma_{n+1}-\gamma_{n+1}, T_{n}}{\Delta t}\right)
\end{aligned}
$$

If we minimize this potential at every material point,

$$
\min _{\substack{\lambda_{n+1} \\ \gamma_{n+1}}} g\left(\lambda_{n+1}, \gamma_{n+1}\right)
$$

we get

$$
\begin{aligned}
& \delta_{\lambda_{n+1}} \rightarrow 0=\frac{\partial A}{\partial \lambda_{i}}\left(\varepsilon^{n+1}, \lambda^{n+1}, \gamma^{n+1}, T^{n}\right)+\frac{\partial D_{t}}{\partial \dot{\lambda}_{i}}\left(\frac{\lambda_{i}^{n+1}-\lambda_{i}^{n}}{\Delta t}, T^{n}\right) \\
& \delta_{\gamma_{n+1}} \rightarrow 0=\frac{\partial A}{\partial \gamma_{\alpha}}\left(\varepsilon^{n+1}, \lambda^{n+1}, \gamma^{n+1}, T^{n}\right)+\frac{\partial D_{p}}{\partial \dot{\gamma}_{\alpha}}\left(\frac{\gamma_{\alpha}^{n+1}-\gamma_{\alpha}^{n}}{\Delta t}, T\right) .
\end{aligned}
$$

We observe that (5.34) \& (5.35) are precisely the implicit discrete time equations (5.30) and (5.31).

We can now update the temperature in a staggered step. The adiabatic heating due to plastic work can be evaluated using the Taylor-Quinney coefficient $\beta$ (also called the inelastic heat fraction coefficient), to which we add (or subtract) the latent heat from the 
transformation, s.t.,

$$
\begin{aligned}
\frac{T^{n+1}-T^{n}}{\Delta t} & =\sum_{\alpha}^{M}\left(\frac{\beta \tau_{\alpha}\left(\frac{\gamma_{\alpha}^{n+1}-\gamma_{\alpha}^{n}}{\Delta t}\right)}{\rho C_{v}}\right)+\sum_{i}^{N}\left(\frac{L\left(\frac{\lambda_{i}^{n+1}-\lambda_{i}^{n}}{\Delta t}\right)}{T_{c} \rho C_{v}}\right) \\
T^{n+1} & =T^{n}+\sum_{\alpha}^{M}\left(\frac{\beta \tau_{\alpha}\left(\gamma_{\alpha}^{n+1}-\gamma_{\alpha}^{n}\right)}{\rho C_{v}}\right)+\sum_{i}^{N}\left(\frac{L\left(\lambda_{i}^{n+1}-\lambda_{i}^{n}\right)}{T_{c} \rho C_{v}}\right)
\end{aligned}
$$

where $\tau_{\alpha}$ is the plastic flow stress, $C_{v}$ is the specific heat (per unit mass), and $L$ is the latent heat of transformation. This assumes that only some fraction $\beta$ of the plastic rate of work contributes to the the heating, and that the thermal contribution from phase transformation is completely dominated by the latent heat.

This discrete time formulation allows us to implement our framework using the Optimal Transport Meshfree (OTM) method $[52,53]$. We first solve $(5.29)-(5.31)$ using the OTM method (where the minimization (5.33) associated with (5.30) and (5.31) is solved locally at the material points), and use (5.36) to update the temperature.

\section{$5.4 \quad$ Finite kinematics}

A major difficulty arises in the formulation of models in finite kinematics due to the geometric nonlinearities and associated finite strain measures. Modern crystal plasticity for-

mulations employ the notion of an intermediate stress-free configuration $\Omega_{p}$, and the well- 
established multiplicative (Kroner-Lee) decomposition of the deformation gradient [47, 61],

$$
\mathbf{F}=\mathbf{F}^{e} \mathbf{F}^{p}
$$

where $\mathbf{F}, \mathbf{F}^{e}, \mathbf{F}^{p}$ are the total, elastic, and plastic deformation gradients, respectively. Such a construction is successful in part because the form of the plastic rate of deformation is assumed to be known from the crystallographic properties; under certain assumptions (cf. e.g. $[54,79])$, the integral of this rate is of the form,

$$
\mathbf{F}^{p}=\mathbf{I}+\sum_{\alpha=1}^{n} \gamma_{\alpha}\left(\mathbf{s}_{\alpha} \otimes \mathbf{m}_{\alpha}\right)
$$

where $\gamma_{\alpha}$ is the scalar slip accumulated on the $\alpha$ slip-system, $\mathbf{s}_{\alpha}$ is the slip direction (unit Burgers vector), and $\mathbf{m}_{\alpha}$ is the unit normal to the slip-plane. A problem arises when we attempt to introduce an equivalent transformation deformation gradient, $\mathbf{F}^{t}$, if we again assume its form is dictated by crystallography: there is clearly an ambiguity in the appropriate order of the multiplicative decomposition, since in general,

$$
\mathbf{F}=\mathbf{F}^{e} \mathbf{F}^{p} \mathbf{F}^{t} \neq \mathbf{F}^{e} \mathbf{F}^{t} \mathbf{F}^{p}
$$

For a given deformation $\mathbf{F}$, arbitrarily choosing one order of the decomposition over another in Eq. 5.39 affects the partitioning of the energy into elastic and inelastic parts.

We propose an extension of the generalized framework to finite kinematics inspired 
by the method of [24], in which an exponential mapping of the update from a model with linearized kinematics is used to extend stress update algorithms to finite kinematics, independent of the material model. This has parallels to a logarthmic (Hencky) strain measure; if we define $\varepsilon$, the finite strain tensor, as

$$
\varepsilon=\frac{1}{2} \log \left(\mathbf{F}^{T} \mathbf{F}\right)
$$

where $\mathbf{F}$ is the deformation gradient, and we again suppose an additive decomposition of the strain,

$$
\varepsilon=\varepsilon_{e}+\varepsilon_{t}+\varepsilon_{p}
$$

then, similar to the case in [24], the multiplicative decomposition would only map to an additive decomposition when the individual components commute (i.e. the components have the same eigenvectors). This is attributable to a fundamental property of the log function itself,

$$
\log (\mathbf{A B})=\log (\mathbf{A})+\log (\mathbf{B}) \Longleftrightarrow \mathbf{A B}=\mathbf{B A}
$$

which essentially implies that, for the decomposition to be valid, the elastic, transformation, and plastic strains must all be in the same "direction" (coaxial) or at least approximately so, though the principal directions of the components need not be isotropic, and the magnitudes of each may differ arbitrarily. Clearly, the transformation and plastic deformation components do not commute, as they are defined by scalar multiples of different crystal- 
lographic systems. We see then that this extension does not actually avoid the problem associated with the ambiguity in the decomposition of the deformation gradient; rather, it makes explicit the assumptions otherwise hidden when blindly choosing one multiplicative decomposition over another - that we are ignoring the difference in the rotation between the different strain components.

The actual method outlined in [24] requires, not that the strain components themselves be coaxial, but rather that the incremental update in plastic deformation (which forms the plastic corrector) be coaxial with the elastic predictor for the right Cauchy-Green deformation tensor. In general, this is rarely so, but as an approximation, we ignore any errors introduced by assuming the quantities commute.

We note that, in the context of crystal plasticity, an increment might be the sum of contributions from several slip systems, consistent with the form of the velocity gradient postulated in [73]. We extend our method by proposing a multiplicative decomposition of the total inelastic deformation gradient and an additive decomposition of the total inelastic velocity gradient,

$$
\mathbf{L}_{i n}=\sum_{\alpha}^{n} \dot{\gamma}_{\alpha}\left(\mathbf{s}_{\alpha} \otimes \mathbf{m}_{\alpha}\right)+\sum_{i}^{n} \dot{\lambda}_{i}\left(\mathbf{U}_{i}-\mathbf{I}\right)
$$

which treats the transformation and plasticity as though they were originating in the same intermediate configuration (analogous to simply increasing the number of slip systems in the plasticity formulation, and treating transformation as being equivalent to another type 
of slip). Following the formulation in [24], this results in an update,

$$
\begin{aligned}
& \mathbf{F}^{n+1}=\mathbf{F}_{e}^{n+1} \mathbf{F}_{i n}^{n+1} \\
& \mathbf{F}_{i n}^{n+1}=\exp \left(\hat{\mathbf{L}}_{i n}^{n+1} \Delta t\right) \mathbf{F}_{i n}^{n} \\
& \mathbf{S}^{n+1}=\mathbf{S}\left(\frac{1}{2} \log \left(\mathbf{C}_{e}^{n+1}\right)\right)
\end{aligned}
$$

where $\hat{\mathbf{L}}_{i n}$ is the modified (symmetric) inelastic deformation gradient afforded by the method of [24], and $\mathbf{S}$ is the second Piola-Kirchhoff stress tensor, defined in the intermediate configuration. We assume $\hat{\mathbf{L}}_{i n}$ to be of the form,

$$
\hat{\mathbf{L}}_{i n}^{n+1}=\sum_{\alpha=1}^{M}\left(\frac{\gamma_{\alpha}^{n+1}-\gamma_{\alpha}^{n}}{\Delta t}\right) \varepsilon_{p}^{\alpha}+\sum_{i=1}^{N}\left(\frac{\lambda_{i}^{n+1}-\lambda_{i}^{n}}{\Delta t}\right) \varepsilon_{t}^{i}
$$

where $\varepsilon_{t}^{i}=\mathbf{U}_{i}-\mathbf{I}$ and $\varepsilon_{p}^{\alpha}=\frac{1}{2}\left(\mathbf{s}_{\alpha} \otimes \mathbf{m}_{\alpha}+\mathbf{m}_{\alpha} \otimes \mathbf{s}_{\alpha}\right)$.

Satisfied with the approximation, this method allows us to compute the increment as in the linear setting, setting $\varepsilon_{e}^{n+1}=\frac{1}{2} \log \left(\mathbf{C}_{e}^{n+1}\right)$ and $\sigma^{n+1}=\mathbf{S}^{n+1}$, and using the kinetic potentials to determine $\lambda_{i}^{n+1}$ and $\gamma_{\alpha}^{n+1}$; we then use this increment to update $\mathbf{F}_{i n}^{n+1}$ via the exponential mapping [65]. 


\section{Chapter 6}

\section{Iron Model: Implementation \& Results}

We demonstrate the suitability of the generalized framework to study two phase transitions

in elemental iron. Iron has many allotropes that are stable at various temperatures and pressures, as shown in Figure 6.1. We note that, unlike SMAs, the phases of iron are not related to a common finite symmetry group (cf. [12]). While still martensitic (i.e. nondiffusional), the transformations in iron are reconstructive, which leads to largely inelastic deformations accompanying transformation.

We emphasize that all results presented in this chapter are merely proof-of-concept; in some instances, non-physical parameters have been used in order to demonstrate the capabilities of the framework. 


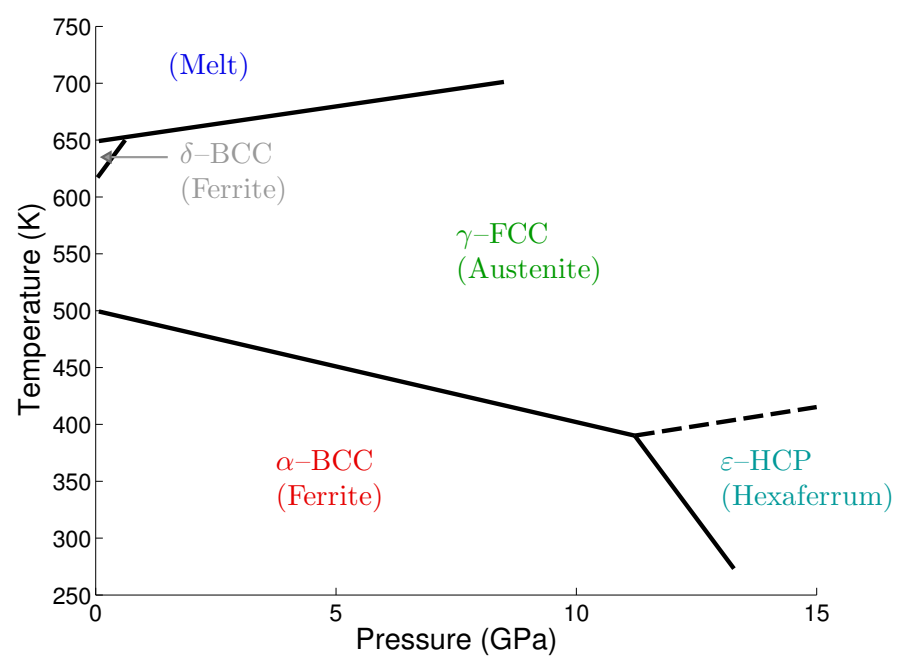

Figure 6.1: Idealized phase diagram for pure iron. (Adapted from [30].)

\section{1 $\alpha-\gamma$ transformation model}

At room temperature and atmospheric conditions, elemental iron has a Body-Centered Cubic (BCC) crystal structure, called ferrite or $\alpha$-iron. For pressures below $11 \mathrm{GPa}$, the crystal structure changes to Face-Centered Cubic (FCC) at a temperature of 500$1000^{\circ} \mathrm{C}$, depending on the pressure (see Figure 6.1). This FCC phase is called $\gamma$-iron, and the transformation is known as the $\alpha-\gamma$ transition $^{1}$. In the context of carbon steels, $\gamma$-phase is traditionally known as austenite, hence austenitic stainless steels; however, in the present formulation, we use the Bain relation between the $\mathrm{BCC}$ lattice and a BodyCentered Tetragonal (BCT) lattice to model the transformation strain, since the FCC lattice is analogue to a BCT lattice (cf. e.g. [11]). Since this description treats the BCC lattice as the parent phase, we shall treat $\alpha$-iron as our "austenitic" phase, and $\gamma$-iron as our "martensitic" phase, and refrain from using the terms ferrite and austenite. Additionally, 
further confusion arises for iron in that the parent $\alpha$-phase is actually the low-temperature phase, and the apparent loss in symmetry is observed at high temperatures, whereas the opposite is true in most SMAs like Nitinol.

\subsection{1 $\gamma$-Transformation systems}

The kinematics for the $\alpha-\gamma$ transition are given in [11],

$$
\mathbf{U}_{1}=\left(\begin{array}{ccc}
\beta & 0 & 0 \\
0 & \alpha & 0 \\
0 & 0 & \alpha
\end{array}\right), \quad \mathbf{U}_{2}=\left(\begin{array}{ccc}
\alpha & 0 & 0 \\
0 & \beta & 0 \\
0 & 0 & \alpha
\end{array}\right), \quad \mathbf{U}_{3}=\left(\begin{array}{ccc}
\alpha & 0 & 0 \\
0 & \alpha & 0 \\
0 & 0 & \beta
\end{array}\right)
$$

where the principle stretches are derived from the crystallographic parameters,

$$
\alpha=\frac{a_{\mathrm{BCT}}}{a_{\mathrm{BCC}}}=\frac{a_{\mathrm{FCC}}}{\sqrt{2} a_{\mathrm{BCC}}}, \quad \beta=\frac{c_{\mathrm{BCT}}}{a_{\mathrm{BCC}}}=\frac{a_{\mathrm{FCC}}}{a_{\mathrm{BCC}}}
$$

Reported measurements for $a_{\mathrm{BCC}} a_{\mathrm{FCC}}$ lead to as much as $5 \%$ variance in the volume of the unit cell (compare [6] to [20]); for the present simulations we have taken values from $[6]$

$$
a_{\mathrm{BCC}}=0.286 \mathrm{~nm}, \quad a_{\mathrm{FCC}}=0.355 \mathrm{~nm}
$$

\footnotetext{
${ }^{1}$ Historically, there has been a so-called $\beta$-iron or $\beta$-ferrite phase, which referred to the paramagnetic form of ferrite above the Curie temperature. This is a second-order phase transition from $\alpha$-ferrite, with the $\beta$-ferrite being crystallographically identical, except for the loss of magnetic domains. At atmospheric pressure, the any difference in the BCC lattice parameter of paramagnetic ferrite and $\alpha$-ferrite is attributable to simple thermal expansion, which is given as $12 \mu \epsilon / \mathrm{K}[20]$. The term $\beta$-iron is now considered obsolete, so we proceed with our use of $\alpha-\gamma$ transition in favor of $\beta-\gamma$ found in some references (e.g. ibid.).
} 


\subsubsection{Plastic slip systems}

In our framework, we assume that any plastic strains can be mapped back to plastic strains in the parent phase. Therefore, we consider only the BCC slip systems in the parent $\alpha$-iron phase, irrespective of the actual phase the material is in when a slip occurs. Since BCC crystals have no truly close-packed planes, there are two families of slip systems with nearly identical activation energies, namely the $\mathbf{m}_{\alpha}=\{110\}$ and $\mathbf{s}_{\alpha}=\langle 111\rangle$ systems, and the $\mathbf{m}_{\alpha}=\{211\}$ and $\mathbf{s}_{\alpha}=\langle 111\rangle$ systems $^{2}$. These are listed in Table 6.1.

\subsubsection{Elastic energy density}

The elastic energy density in Eq. (5.10) is assumed to be of the form

$$
W^{e}\left(\varepsilon^{e}\right)=f\left(\operatorname{tr}\left(\varepsilon^{e}\right), T\right)+W^{e, \operatorname{dev}}\left(\varepsilon^{e, \operatorname{dev}}, T\right)
$$

We use an empirical, quadratic equation of state $f\left(\operatorname{tr}\left(\varepsilon^{e}\right), T\right)$, with a bulk modulus $\kappa=$ 170.9GPa. In the absence of experimental curves for the deviatoric elastic strain energy density, we approximate the general function with a Taylor series expansion in temperature and deviatoric strain, s.t.

$$
W^{e, \operatorname{dev}}\left(\varepsilon^{e, \operatorname{dev}}, T\right)=\frac{1}{2} \varepsilon^{e, \operatorname{dev}} \cdot \mathrm{C}^{\operatorname{dev}}(T) \cdot \varepsilon^{e, \operatorname{dev}}
$$

\footnotetext{
${ }^{2}$ Additionally, the family of $\mathbf{m}_{\alpha}=\{312\}$ and $\mathbf{s}_{\alpha}=\langle 111\rangle$ is sometimes considered as a possible slip system in BCC crystals. These are enumerated in [63].
} 
84

Table 6.1: Slip systems for BCC crystals (unnormalized).

Source: [28]

\begin{tabular}{|c|c|c|}
\hline Slip System & Plane Normal $\mathbf{m}_{\alpha}$ & Slip Direction $\mathbf{s}_{\alpha}$ \\
\hline $1 \mathrm{C}$ & $\left(\begin{array}{lll}0 & 1 & 1\end{array}\right)$ & $\pm\left[\begin{array}{lll}\overline{1} & \overline{1} & 1\end{array}\right]$ \\
\hline $1 \mathrm{D}$ & $\left(\begin{array}{llll}0 & 1 & 1\end{array}\right)$ & $\pm\left[\begin{array}{lll}1 & \overline{1} & 1\end{array}\right]$ \\
\hline $1^{\prime} \mathrm{C}$ & $(2 \overline{1} 1)$ & $\pm\left[\begin{array}{lll}\overline{1} & \overline{1} & 1\end{array}\right]$ \\
\hline $1^{\prime \prime} \mathrm{D}$ & $(\overline{2} \overline{1} 1)$ & $\pm\left[\begin{array}{lll}1 & \overline{1} & 1\end{array}\right]$ \\
\hline $2 \mathrm{~A}$ & $(0 \overline{1} 1)$ & $\pm\left[\begin{array}{lll}1 & 1 & 1\end{array}\right]$ \\
\hline $2 \mathrm{~B}$ & $(0 \overline{1} 1)$ & $\pm\left[\begin{array}{lll}1 & 1 & 1\end{array}\right]$ \\
\hline $2^{\prime} \mathrm{A}$ & $(211)$ & $\pm\left[\begin{array}{lll}1 & 1 & 1\end{array}\right]$ \\
\hline $2^{\prime \prime} \mathrm{B}$ & $(\overline{2} 11)$ & $\pm\left[\begin{array}{lll}1 & 1 & 1\end{array}\right]$ \\
\hline $3 \mathrm{~A}$ & $\left(\begin{array}{lll}1 & 0 & 1\end{array}\right)$ & $\pm\left[\begin{array}{lll}1 & 1 & 1\end{array}\right]$ \\
\hline $3 \mathrm{C}$ & $\left(\begin{array}{lll}1 & 0 & 1\end{array}\right)$ & $\pm\left[\begin{array}{lll}\overline{1} & \overline{1} & 1\end{array}\right]$ \\
\hline $3^{\prime} \mathrm{A}$ & $(\overline{1} \overline{2} 1)$ & $\pm\left[\begin{array}{lll}1 & 1 & 1\end{array}\right]$ \\
\hline $3^{\prime \prime} \mathrm{C}$ & $(\overline{1} 21)$ & $\pm\left[\begin{array}{lll}\overline{1} & \overline{1} & 1\end{array}\right]$ \\
\hline $4 \mathrm{~B}$ & $\left(\begin{array}{lll}1 & 0 & 1\end{array}\right)$ & $\pm\left[\begin{array}{lll}1 & 1 & 1\end{array}\right]$ \\
\hline $4 \mathrm{D}$ & $\left(\begin{array}{lll}\overline{1} & 0 & 1\end{array}\right)$ & $\pm\left[\begin{array}{lll}1 & \overline{1} & 1\end{array}\right]$ \\
\hline $4^{\prime} \mathrm{B}$ & $(1 \overline{2} 1)$ & $\pm\left[\begin{array}{lll}1 & 1 & 1\end{array}\right]$ \\
\hline $4^{\prime \prime} \mathrm{D}$ & $\left(\begin{array}{lll}1 & 2 & 1\end{array}\right)$ & 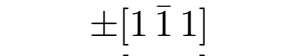 \\
\hline $5 \mathrm{~B}$ & $(\overline{1} 10)$ & $\pm\left[\begin{array}{lll}1 & 1 & 1\end{array}\right]$ \\
\hline $5 \mathrm{C}$ & $(\overline{1} 10)$ & $\pm\left[\begin{array}{lll}\overline{1} & \overline{1} & 1\end{array}\right]$ \\
\hline $5^{\prime} \mathrm{B}$ & $(11 \overline{2})$ & $\pm\left[\begin{array}{lll}1 & 1 & 1\end{array}\right]$ \\
\hline $5^{\prime \prime} \mathrm{C}$ & $(\overline{1} \overline{1} \overline{2})$ & $\pm\left[\begin{array}{lll}\overline{1} & \overline{1} & 1\end{array}\right]$ \\
\hline $6 \mathrm{~A}$ & $(110)$ & $\pm\left[\begin{array}{lll}\overline{1} & 1 & 1\end{array}\right]$ \\
\hline $6 \mathrm{D}$ & $(110)$ & $\pm\left[\begin{array}{lll}1 & \overline{1} & 1\end{array}\right]$ \\
\hline $6^{\prime} \mathrm{A}$ & $(\overline{1} 1 \overline{2})$ & $\pm\left[\begin{array}{lll}1 & 1 & 1\end{array}\right]$ \\
\hline $6^{\prime \prime} \mathrm{D}$ & $(1 \overline{1} \overline{2})$ & $\pm\left[\begin{array}{lll}1 & \overline{1} & 1\end{array}\right]$ \\
\hline
\end{tabular}

where $\mathrm{C}^{\mathrm{dev}}(T)$ is the deviatoric projection of an anisotropic elastic modulus $\mathrm{C}(T)$. A first order expansion of $\mathrm{C}^{\text {dev }}$ in temperature gives

$$
\mathrm{C}^{\mathrm{dev}}(T)=\mathrm{C}_{0}^{\mathrm{dev}}-\mathrm{C}_{1}^{\mathrm{dev}} T
$$


The components of $\mathrm{C}_{0}^{\mathrm{dev}}$ are computed from the total stiffness $\mathrm{C}_{0}$ obeying cubic symmetry, given by [93] (citing Simmons \& Wang, 1971)

$$
\mathrm{C}_{0}^{11}=236.9 \mathrm{GPa}, \quad \mathrm{C}_{0}^{12}=140.6 \mathrm{GPa}, \quad \mathrm{C}_{0}^{44}=116.0 \mathrm{GPa},
$$

and similarly for $\mathrm{C}_{1}^{\text {dev }}$ from $\mathrm{C}_{1}$,

$$
\mathrm{C}_{1}^{11}=0.012 \cdot \mathrm{C}_{0}^{11} \mathrm{GPa} / \mathrm{K}, \quad \mathrm{C}_{1}^{12}=0.004 \cdot \mathrm{C}_{0}^{12} \mathrm{GPa} / \mathrm{K}, \quad \mathrm{C}_{1}^{44}=0.008 \cdot \mathrm{C}_{0}^{44} \mathrm{GPa} / \mathrm{K}
$$

\subsubsection{Transformation stored energy}

The transformation stored energy density, or chemical potential, determines the relative stability of the $\alpha$ and $\gamma$ phases. In iron, it prefers the FCC- $\gamma$ phase above the critical temperature, and the BCC- $\alpha$ phase below. We assume,

$$
W^{t}(\lambda, T)=w(T) \sum_{i=1}^{3} \lambda_{i}
$$

with

$$
w(T)=L \frac{T-T_{c}}{T_{c}}
$$


where $L$ is the latent heat of transformation, given in $[20]$ as $0.900 \mathrm{~kJ} / \mathrm{mol}(16.11 \mathrm{~kJ} / \mathrm{kg}$, 126.9 $\left.\mathrm{MJ} / \mathrm{m}^{3}\right)$ at $T_{c}=910^{\circ} \mathrm{C}$.

\subsubsection{Pastic work-hardening}

We choose a simple power-law to model the hardening associated with plastic slip, which we scale by a decoupled power-law dependence on temperature, s.t.

$$
W^{p}(\gamma, T)=\left(\sum_{\alpha=1}^{N} \tau_{\alpha}^{c 0} \gamma_{\alpha}+\frac{n}{n+1} \tau_{0} \gamma_{0}\left(\frac{\gamma_{e q}}{\gamma_{0}}\right)^{\frac{n+1}{n}}\right)\left(1-\left(\frac{T-T_{0}}{T_{m}-T_{0}}\right)^{a}\right)
$$

where $\gamma_{e q}=\sum_{\beta} \gamma_{\beta}, \tau_{\alpha}^{c 0}$ is the initial yield stress, $T_{0}$ is the reference temperature (usually room temperature), and $T_{m}$ is the melt temperature (where the material loses all strength); these parameters were chosen to be,

$$
\begin{aligned}
& \tau_{\alpha}^{c 0}=10(\mathrm{MPa}), \quad \tau_{0}=100(\mathrm{MPa}), \quad \gamma_{0}=1, \\
& n=10, \quad a=1, \\
& T_{0}=295(\mathrm{~K}), \quad T_{m}=1811(\mathrm{~K}) .
\end{aligned}
$$

We note that, for the more general form $\gamma_{e q}=\sqrt{a_{\alpha \beta} \gamma_{\alpha} \gamma_{\beta}}$, this potential is capable of modelling latent hardening; however, many numerical difficulties are encountered as such a potential leads to nonconvexities in the energy landscape (cf. e.g. []). 


\subsubsection{Kinetic laws}

For transformation, we choose a broken parabolic potential to give stick slip behavior, s.t.

$$
D_{t}(\dot{\lambda})=\sum_{i=1}^{M}\left(\frac{1}{2} r_{2} \dot{\lambda}_{i}^{2}+r_{1}\left|\dot{\lambda}_{i}\right|+r_{0}\right)
$$

where

$$
r_{2}=100(\mathrm{MPa} \cdot \mathrm{s}), \quad r_{1}=L / 10=12.7(\mathrm{MPa}), \quad r_{0}=0
$$

For plasticity, we slightly relax the notion of the stick-slip kinetic laws (borrowed from transformation kinetics) in favor of a power law, which gives regularity at the origin. In plasticity, this results in a creep-like behavior, which can be minimized (or amplified) by an appropriate choice of the rate hardening exponent. We assume the general form,

$$
D_{p}(\dot{\gamma})=\sum_{\alpha=1}^{N} \tilde{\tau}_{\alpha}^{c 0} \dot{\gamma}_{\alpha}+\frac{m}{m+1} \tilde{\tau}_{0}^{\alpha} \dot{\gamma}_{0}\left(\frac{\dot{\gamma}_{\alpha}}{\dot{\gamma}_{0}}\right)^{\frac{m+1}{m}}
$$

where

$$
\begin{array}{ll}
\tilde{\tau}_{\alpha}^{c 0}=0(\mathrm{MPa}), & \tilde{\tau}_{0}^{\alpha}=100(\mathrm{MPa}), \\
\dot{\gamma}_{0}=1\left(\mathrm{~s}^{-1}\right), & n=100 .
\end{array}
$$




\subsubsection{Temperature update}

For the temperature update in Equation (5.26)

$$
\Delta T=\sum_{\alpha}^{M}\left(\frac{\beta \tau_{\alpha} \Delta \gamma_{\alpha}}{\rho C_{v}}\right)+\sum_{i}^{N}\left(\frac{L \Delta \lambda_{i}}{T_{c} \rho C_{v}}\right)
$$

we choose $\beta=0.9, \tau_{\alpha}=\sigma \cdot \varepsilon_{\alpha}^{p, 0}$ (such that the plastic rate of work is given by $\tau_{\alpha} \dot{\gamma}_{\alpha}$ ), and the specific heat per unit volume $\rho C_{v}=3.5 \frac{\mathrm{MJ}}{\mathrm{m}^{3} \cdot \mathrm{K}}$.

\subsubsection{Constraint enforcement}

In order to satisfy the constraint on $\lambda$ Eq. (5.4), we augment the potential in Eq. (5.10) with a high-order polynomial,

$$
W^{\text {penalty }}(\lambda)=\left(1-\sum_{i=1}^{N} \lambda_{i}\right)^{-p}, \quad p=12
$$

which stays very small when $\lambda$ is small, and grows rapidly as the constraint is approached. It should be noted that this modifies the driving force slightly, through a straightforward differentiation of the total pseudo-potential. This adds a very small amount of hardening to the initial behavior of the phase transformation, until saturation nears; in general, it may cause the transformation to saturate slightly below unity. Such a penalty term is

numerically effective so long as the loading step-size is small enough that $\sum_{i=1}^{N} \lambda_{i}$ never exceeds unity during intermediate iterations. 
As previously mentioned, transformation in iron is accompanied by unrecoverable deformations. For ease of implementation, we introduce the additional constraint,

$$
\dot{\lambda_{i}}, \geq 0
$$

which effectively prevents reverse transformation. This limits the behavior of the material such that, once it transforms to the $\gamma$-phase, it can never return to the parent $\alpha$-phase. Such "frozen" kinetics make the formulation for transformation identical to that of plasticity, which also has the constraint $\dot{\gamma_{\alpha}} \geq 0$ when positive and negative slip directions are counted as separate systems. This constraint is in no way inherent to the generalized framework, and was merely implemented for algorithmic convenience (described below); in principle, an explicit interaction term could be added to Equation (5.10), and the nature of the unrecoverable strains could be investigated using the generalized framework.

\subsubsection{Active slip and transformation systems}

In the iron model, not all slip or transformation systems are active during a given loading

step, and the set of active systems may change from step to step. The identification of active constraints is non-trivial, as many of the slip and transformation systems are not linearly independent. To identify the optimal combination of active constraints, we use the method described in [25], in which we compute a fully elastic predictor, and identify the transformation or slip system with the largest driving force. If the driving force is 
above the critical "stick" force in the stick-slip kinetic law, we compute the increment in slip (or transformation) via Newton-Raphson and recheck the driving forces. If any driving force is still over the critical value, we add the next most overloaded system to the active constraints, and simultaneously solve for the increment on each system. We continue this procedure, adding and removing constraints from the active set, until all driving forces are at (or below) their respective critical values.

This method, while giving the optimal combination, is known to suffer from oscillations and slow convergence. For the $\alpha-\gamma$ iron simulations that follow, we observed this to be problematic only in isolated nodes near constrained surfaces. When a suitable combination of active systems could not be determined after a reasonable number of iterations $(100 \times$ $\left.\left[N^{t}+N^{p}\right]=2700\right)$, the algorithm computed an explicit update and proceeded to the next loading step.

\subsection{Taylor impact}

The Taylor impact test [91] is a widely used experimental technique for characterizing the dynamic response of materials. The test consists of impacting a cylindrical projectile perpendicularly against a rigid, planar surface, and measuring (postmortem) the length of the intact (undeformed) portion of the bar. If the initial velocity of the bar is known, this length can be used to compute a lower bound the maximum deceleration of the bar, and thereby compute a lower bound on the dynamic yield strength. Starting with [94], 
91

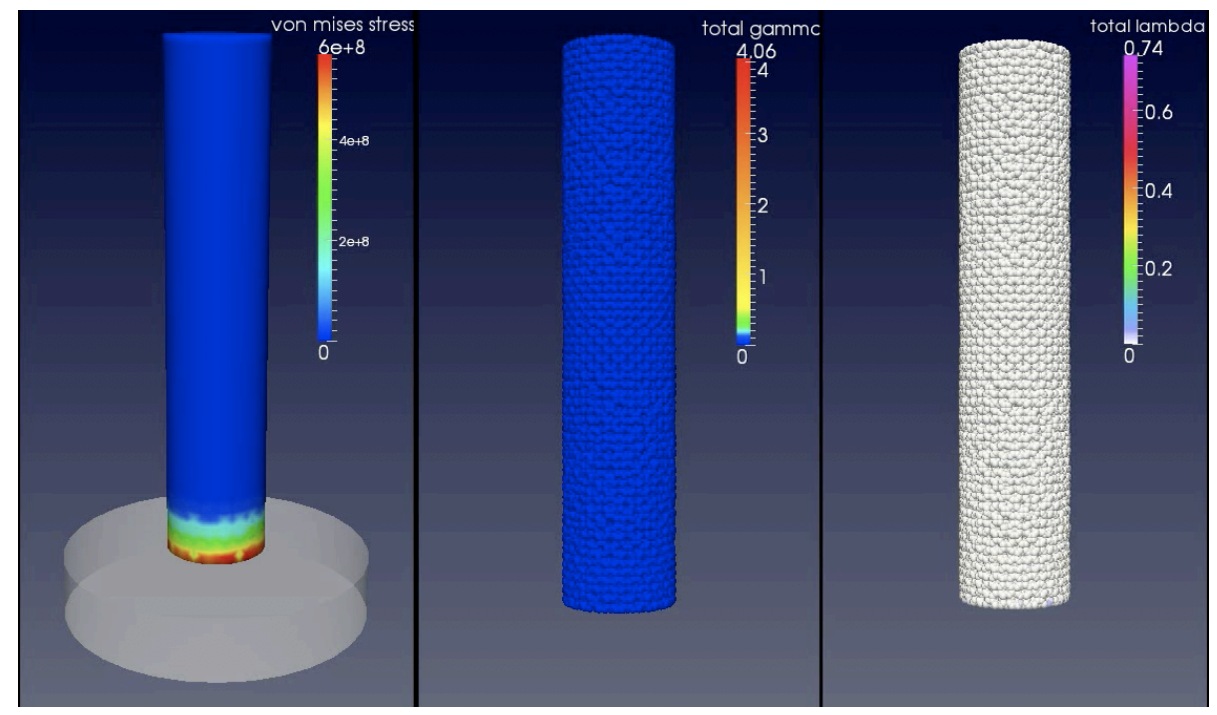

Figure 6.2: Taylor impact simulation of an iron bar for $\alpha-\gamma$ transformation at $227 \mathrm{~m} / \mathrm{s}$ (from scalability study). (Step 2.) The left field shows the von Mises stress; the center field shows the accumlated slip, summed over all systems; the right field shows the total martensite volume fraction, summed over all variants. We see the elastic precursor propagating upwards.

numerical simulations of the Taylor impact test have become a popular means of evaluating new constitutive laws.

\subsubsection{Simulation results}

To demonstrate the suitability of the framework to large-scale numerical investigations, we simulate a Taylor impact experiment of an iron bar hitting a rigid wall at $227 \mathrm{~m} / \mathrm{s}$. The implementation makes use of a parallelized OTM solver [52] coded in C++ and OpenMPI. We use a "gumball" model for the bar, where each material point is assigned a random 3-D orientation representing an individual grain, such that the grains are severely underresolved; explicit dynamics are computed for the bar, and the constitutive response at each 


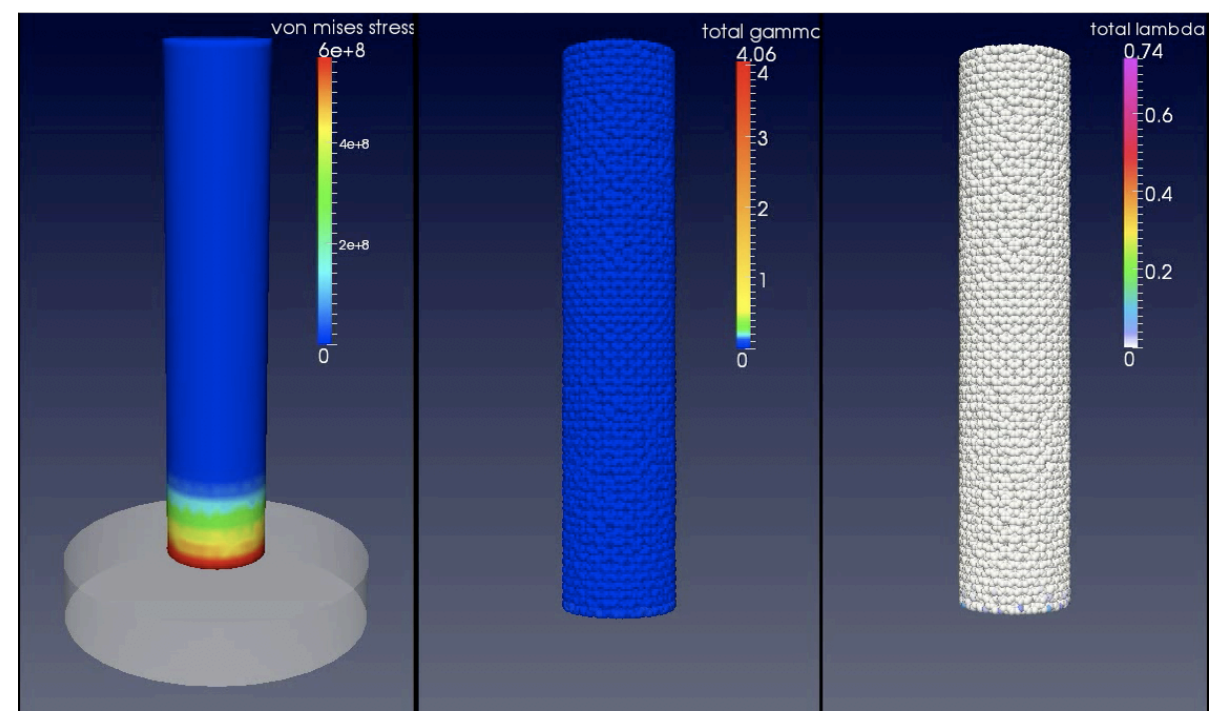

Figure 6.3: Taylor impact simulation of an iron bar for $\alpha-\gamma$ transformation at $227 \mathrm{~m} / \mathrm{s}$ (from scalability study). (Step 3.) Initiation of plasticity and transformation is seen at the base of the specimen.

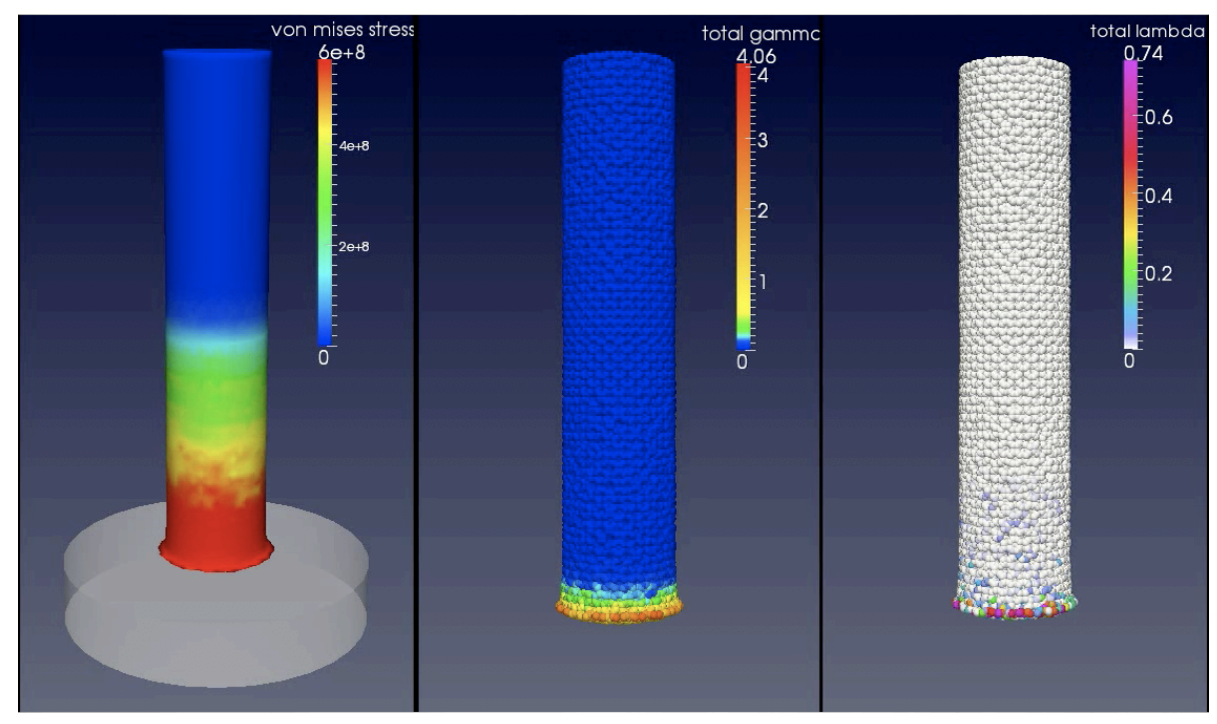

Figure 6.4: Taylor impact simulation of an iron bar for $\alpha-\gamma$ transformation at $227 \mathrm{~m} / \mathrm{s}$ (from scalability study). (Step 9.) We see the onset of the inelastic wave, propagating upwards. 


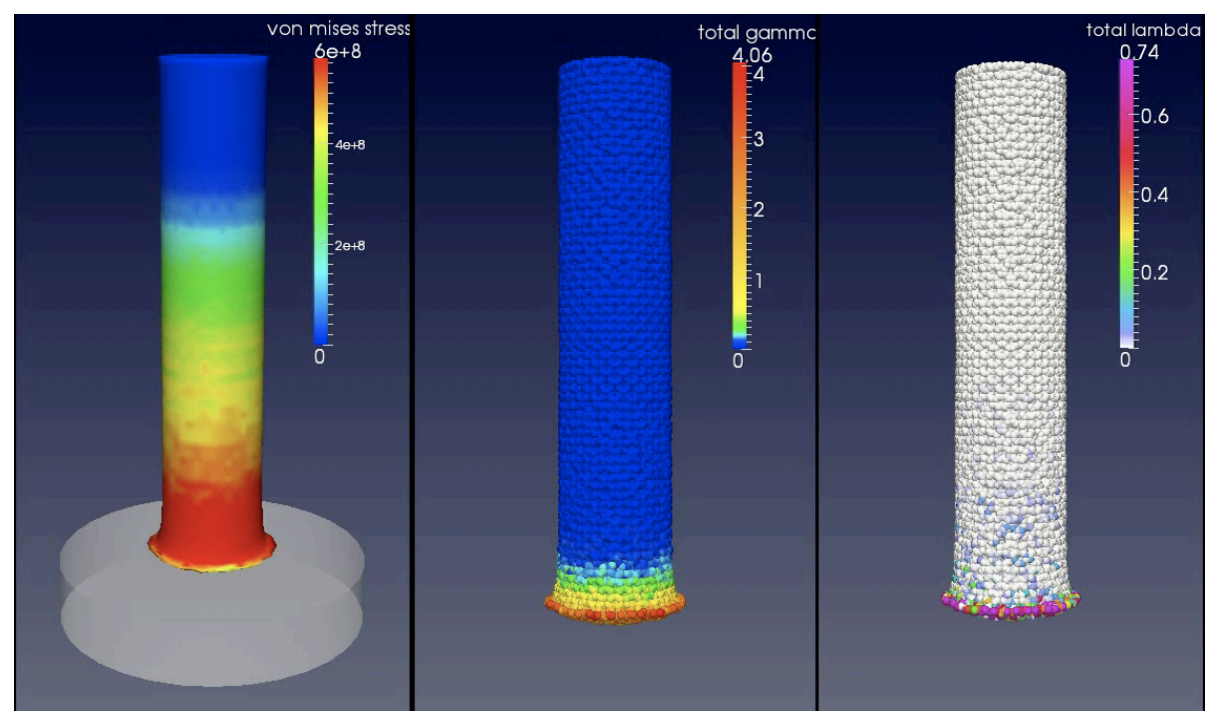

Figure 6.5: Taylor impact simulation of an iron bar for $\alpha-\gamma$ transformation at $227 \mathrm{~m} / \mathrm{s}$ (from scalability study). (Step 11.) The inelastic wave continues to propagate. We note that isolated transformation is visible ahead of the plastic wave, but that extensive transformation lags the plastic wavefront.

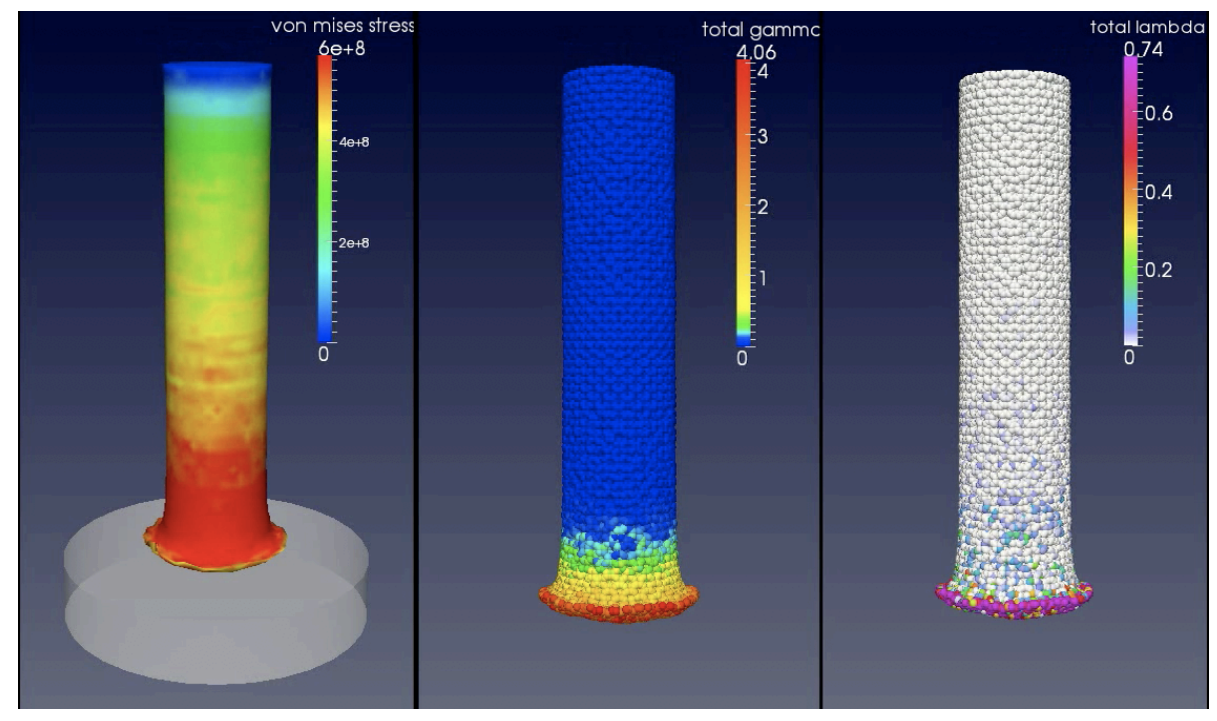

Figure 6.6: Taylor impact simulation of an iron bar for $\alpha-\gamma$ transformation at $227 \mathrm{~m} / \mathrm{s}$ (from scalability study). (Step 16.) We see the elastic wave reaching the end of the bar. 


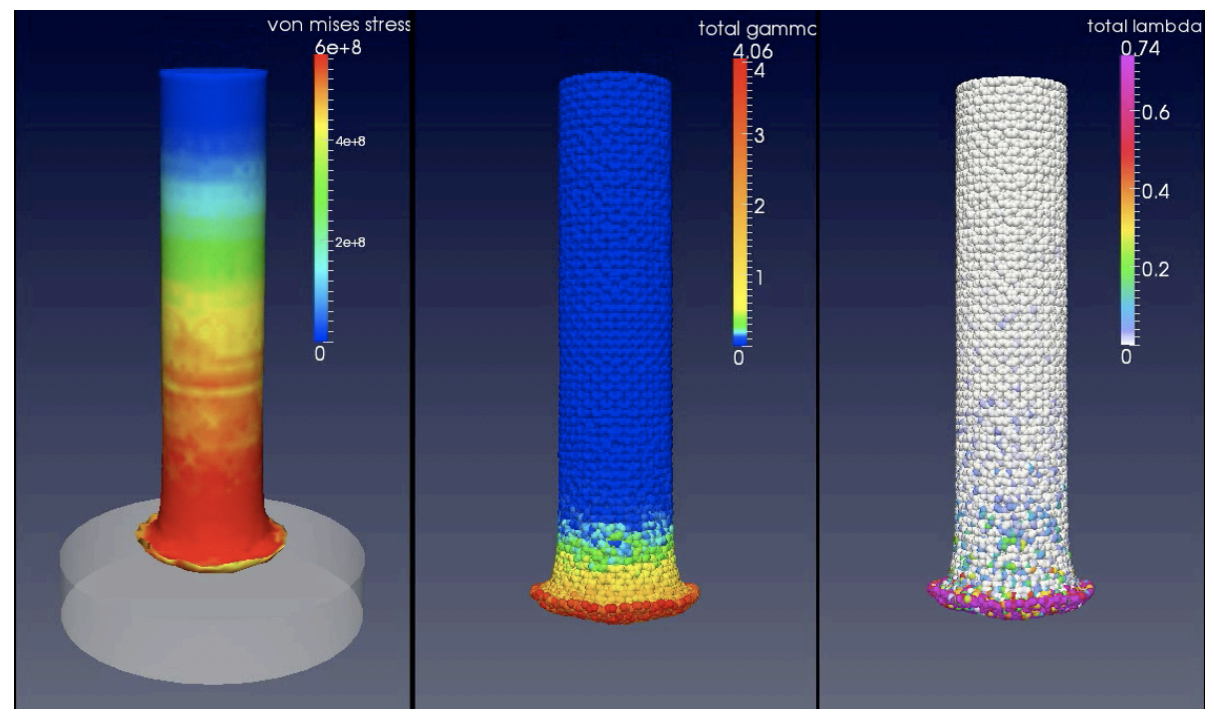

Figure 6.7: Taylor impact simulation of an iron bar $\alpha-\gamma$ transformation at $227 \mathrm{~m} / \mathrm{s}$ (from scalability study). (Step 21.) The elastic wave has reflected as a tensile wave, propagating downwards.

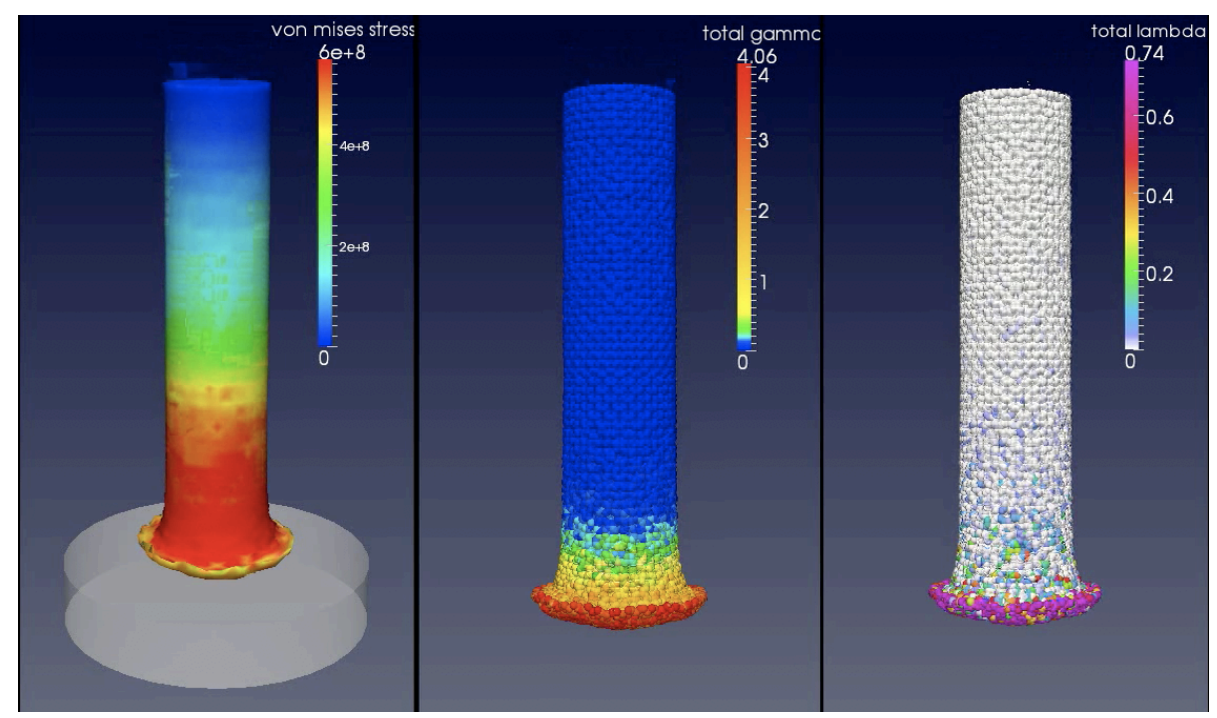

Figure 6.8: Taylor impact simulation of an iron bar $\alpha-\gamma$ transformation at $227 \mathrm{~m} / \mathrm{s}$ (from scalability study). We see the reflected wave tensile wave meeting the plastic wave front. 
material point is determined locally using the framework developed above. In all, 125,309 randomly oriented grains were used to model a $32.4 \mathrm{~mm}$ cylindrical rod with a radius of $3.2 \mathrm{~mm}$.

In Figure 6.2, we see the von Mises stress depicted alongside the slip (summed over all systems) and the martensitic volume fraction (summed over all variants) near the beginning of the simulation; the upwards-travelling elastic precursor is visible in stress plot, with no evolution in the kinetic variables. In each of the figures, each output step corresponds to 50 simulation time steps. In Figure 6.3, a few grains at the bottom of the bar have just begun to plastify and transform. In Figures $6.4 \& 6.5$, we see an inelastic wave begin to spread; we note that isolated spots of transformation lead the plastic wavefront, appearing closer to the elastic wavefront, but that regions of extensive transformation appear to lag the plastic wave. In Figure 6.6, we see the elastic wave reach the top, and reflect back down the bar as a tensile wave in Figures $6.7 \& 6.8$.

\subsubsection{Scalability study}

To test the scalability of the framework, we run the simulation for the Taylor impact test on Caltech's Shared Heterogeneous Computing (SHC) cluster, varying the number of MPI tasks. For this batch of simulations, each material point represents a grain in the microscale, and is assigned a random 3-D orientation. The crystal plasticity and phase transformation model is employed to simulate the dynamic response of each grain. In all, 125,309 grains are used for each simulation. As can be seen in Figure 6.9, the 


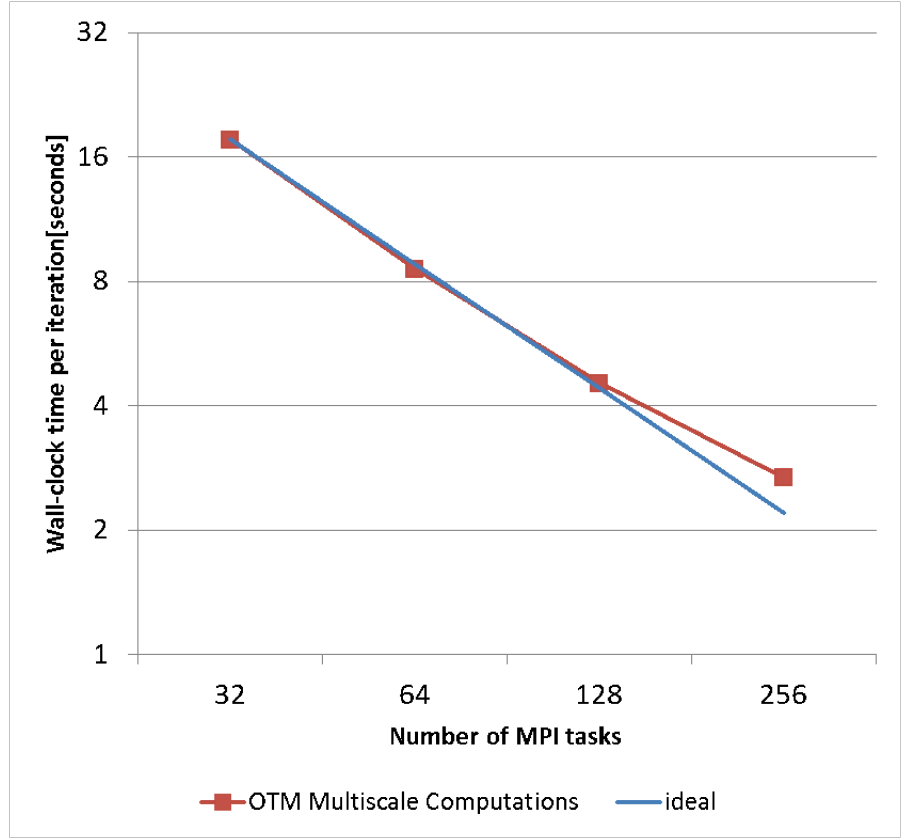

Figure 6.9: Scalability study of the generalized framework for a Taylor anvil impact simulation of an iron bar. [Figure courtesy of Dr. B. Li.] 
implementation achieves the ideal linear speed-up (on a log plot) through 128 tasks (using rank-1 tasks, i.e. one processor per task); at 256 tasks, we observe a very slight decrease from optimal performance. It should be noted that, for the scalability study, some of the material parameters were changed to tax the algorithm, guaranteeing that many slip and transformation systems were active within a single material point from the very beginning

of the simulations. In particular, the latent heat was lowered to $L=1600 \mathrm{~J} / \mathrm{m}^{3}$, and the yield stress was lowered to $\tau_{\alpha}=10 \mathrm{MPa}$.

\section{$6.3 \quad \varepsilon$-iron and $\alpha$-twinning}

At moderately low temperatures $\left(<500^{\circ} \mathrm{C}\right)$ and extremely high pressures $(>10 \mathrm{GPa})$, $\alpha$-iron is observed to transform to a Heaxgonal Close-Packed (HCP) crystal structure, socalled $\varepsilon$-iron or hexaferrum. The kinematics of this transformation result in six martensitic 
variants (cf. [50], and refs. therein),

$$
\begin{array}{rlrl}
\mathbf{U}^{1} & =\left(\begin{array}{ccc}
f+g & -f+g & 0 \\
-f+g & f+g & 0 \\
0 & 0 & h
\end{array}\right), & \mathbf{U}^{2}=\left(\begin{array}{ccc}
f+g & f-g & 0 \\
f-g & f+g & 0 \\
0 & 0 & h
\end{array}\right), \\
\mathbf{U}^{3}=\left(\begin{array}{ccc}
f+g & 0 & f-g \\
0 & h & 0 \\
f-g & 0 & f+g
\end{array}\right), & \mathbf{U}^{4}=\left(\begin{array}{ccc}
f+g & 0 & -f+g \\
0 & h & 0 \\
-f+g & 0 & f+g
\end{array}\right), \\
\mathbf{U}^{5}=\left(\begin{array}{ccc}
f \\
h & 0 & 0 \\
0 & f+g & -f+g \\
0 & -f+g & f+g
\end{array}\right), & \mathbf{U}^{6}=\left(\begin{array}{lll}
h & 0 & 0 \\
0 & f+g & f-g \\
0 & f-g & f+g
\end{array}\right)
\end{array}
$$

where $f=\frac{3}{4 \sqrt{2}}, g=\sqrt{\frac{3}{32}} \frac{c}{a}, h=\frac{\sqrt{3}}{2}$. For iron, the ratio between the lattice parameters $c / a$ appears to be roughly 1.58 [ibid., Table 5]. It should be noted that these Bain strains are for the ideal rigid-sphere geometry, and have not been corrected for the difference between BCC and HCP lattice parameters; therefore, the volumetric strain associated with the HCP transformation in the results that follow is overestimated (cf. [56]). (In the future, this could be corrected using a different definition for the Bain strains.)

Two complications arise in $\alpha-\varepsilon$ transformation. First, we can no logner neglect the possibility of deformation twinning in the $\alpha$-iron, as the critical stress for twinning in the $\mathrm{BCC}$ crystal is on par with that of the transformation stress for the $\varepsilon$-iron. Second, due 
to the symmetries of the two crystal classes involved, the possibility of the martensitic $\varepsilon$ iron reverse transforming into a twin of its original parent phase then transforming again, leads to an energy landscape which, in the absence of other processes, could potentially accommodate an arbitrary (infinite) deformation at constant stress once the transformation plateau is reached (cf. $[50,13])$, which is apparently non-physical. This later issue does not affect our model, as effects from the kinetic laws naturally limit this process.

We make an initial attempt to account for twinning in the parent phase in the following way. First, we see that analogous to the BCC-FCC transformation, we can equally describe 
the kinematics of a BCC-BCC-twin as a transformation using a Bain strain approach,

$$
\begin{aligned}
& \mathbf{U}^{1}=\frac{1}{4}\left(\begin{array}{ccc}
1 & 3 & 2 \\
3 & 1 & -2 \\
-3 & 3 & -2
\end{array}\right), \quad \mathbf{U}^{2}=\frac{1}{4}\left(\begin{array}{ccc}
1 & 3 & -2 \\
3 & 1 & 2 \\
3 & -3 & -2
\end{array}\right), \quad \mathbf{U}^{3}=\frac{1}{4}\left(\begin{array}{ccc}
1 & -3 & 2 \\
-3 & 1 & 2 \\
-3 & -3 & -2
\end{array}\right), \\
& \mathbf{U}^{4}=\frac{1}{4}\left(\begin{array}{ccc}
1 & -3 & -2 \\
-3 & 1 & -2 \\
3 & 3 & -2
\end{array}\right), \quad \mathbf{U}^{5}=\frac{1}{4}\left(\begin{array}{ccc}
1 & -2 & -3 \\
3 & -2 & 3 \\
-3 & -2 & 1
\end{array}\right), \quad \mathbf{U}^{6}=\frac{1}{4}\left(\begin{array}{ccc}
1 & 2 & -3 \\
-3 & -2 & -3 \\
-3 & 2 & 1
\end{array}\right), \\
& \mathbf{U}^{7}=\frac{1}{4}\left(\begin{array}{ccc}
1 & -2 & 3 \\
3 & -2 & -3 \\
3 & 2 & 1
\end{array}\right), \quad \mathbf{U}^{8}=\frac{1}{4}\left(\begin{array}{ccc}
1 & 2 & 3 \\
-3 & -2 & 3 \\
3 & -2 & 1
\end{array}\right), \quad \mathbf{U}^{9}=\frac{1}{4}\left(\begin{array}{ccc}
-2 & -3 & 3 \\
2 & 1 & 3 \\
-2 & 3 & 1
\end{array}\right), \\
& \mathbf{U}^{10}=\frac{1}{4}\left(\begin{array}{ccc}
-2 & 3 & -3 \\
-2 & 1 & 3 \\
2 & 3 & 1
\end{array}\right), \quad \mathbf{U}^{11}=\frac{1}{4}\left(\begin{array}{ccc}
-2 & -3 & -3 \\
2 & 1 & -3 \\
2 & -3 & 1
\end{array}\right), \quad \mathbf{U}^{12}=\frac{1}{4}\left(\begin{array}{ccc}
-2 & 3 & 3 \\
-2 & 1 & -3 \\
-2 & -3 & 1
\end{array}\right)
\end{aligned}
$$

We introduce a new kinetic variable $\alpha$, which represents the volume fraction at a material point of the BCC-twins listed above, s.t.,

$$
\sum_{i=1}^{6} \lambda_{i}+\sum_{j=1}^{12} \alpha_{j}=1, \quad 0 \leq \lambda_{i}, \alpha_{j} \leq 1, \quad \dot{\lambda_{i}}, \dot{\alpha}_{j} \geq 0
$$




\subsubsection{Constitutive law}

Since twinning in the parent phase has no associated chemical energy (i.e. the wells are all at the same height), there is no additional energy density potential associated with $\alpha$ (or equally, we could prescribe the same law as for $\lambda$, and just set the latent heat $\mathcal{L}=0$ ),

$$
W^{w}(\alpha)=\mathcal{L}^{w}(T) \sum_{j=1}^{12} \alpha_{j}, \quad \mathcal{L}^{w} \equiv 0
$$

$W^{t}(\lambda, T)$ is left essentially unchanged, except for the difference in material parameters for the $\varepsilon$-transformation [81],

$$
W^{t}(\lambda)=L \frac{T-T_{c}}{T_{c}} \sum_{i=1}^{6} \lambda_{i}, \quad L=11.47 \frac{\mathrm{GJ}}{\mathrm{m}^{3}}, \quad T_{c}=800 \mathrm{~K}
$$

We now choose the same power-law form for the transformation and twinning kinetic law as we used in plasticity. Here, the creep-like behavior is undesirable, but is still an acceptable approximation to stick-slip behavior. We choose

$$
D_{t}(\dot{\lambda})=\sum_{i=1}^{N} \hat{\tau}_{i}^{c 0} \dot{\lambda}_{i}+\frac{r}{r+1} \hat{\tau}_{0}^{i} \dot{\lambda}_{0}\left(\frac{\dot{\lambda}_{i}}{\dot{\lambda}_{0}}\right)^{\frac{r+1}{r}}
$$


where

$$
\begin{array}{ll}
\hat{\tau}_{i}^{c 0}=L / 10=1.5(\mathrm{GPa}), & \hat{\tau}_{0}^{i}=125(\mathrm{MPa}), \\
\dot{\lambda}_{0}=1, & r=10
\end{array}
$$

We note that the parameter $\hat{\tau}_{i}^{c 0}$ controls the hysteresis of the transformation plateau: the nominal plateau is completely determined by the storage function $W^{t}(\lambda, T)$ and the crystallographic properties, but the critical projected stress is then shifted by $\hat{\tau}_{i}^{c 0}$. We prescribe a kinetic law for $\alpha$ nearly identical to that for $\lambda$, but choose the parameters so that the critical stress for the onset of twinning is on par with that for transformation. For twinning,

$$
D_{w}(\dot{\alpha})=\sum_{j=1}^{N} \hat{\tau}_{j}^{c 0} \dot{\alpha}_{j}+\frac{s}{s+1} \hat{\hat{\tau}}_{0}^{j} \dot{\alpha}_{0}\left(\frac{\dot{\alpha}_{j}}{\dot{\alpha}_{0}}\right)^{\frac{s+1}{s}}
$$

where

$$
\begin{array}{ll}
\hat{\hat{\tau}}_{j}^{c 0}=\hat{\tau}_{i}^{c 0}+L^{\lambda} \frac{T_{0}-T_{c}}{T_{c}}(\mathrm{MPa}), & \hat{\hat{\tau}}_{0}^{j}=100(\mathrm{MPa}), \\
\dot{\alpha}_{0}=1, & s=100
\end{array}
$$

We assume all other elastic and plastic properties to be the same as in the $\alpha-\gamma$ transformation. 


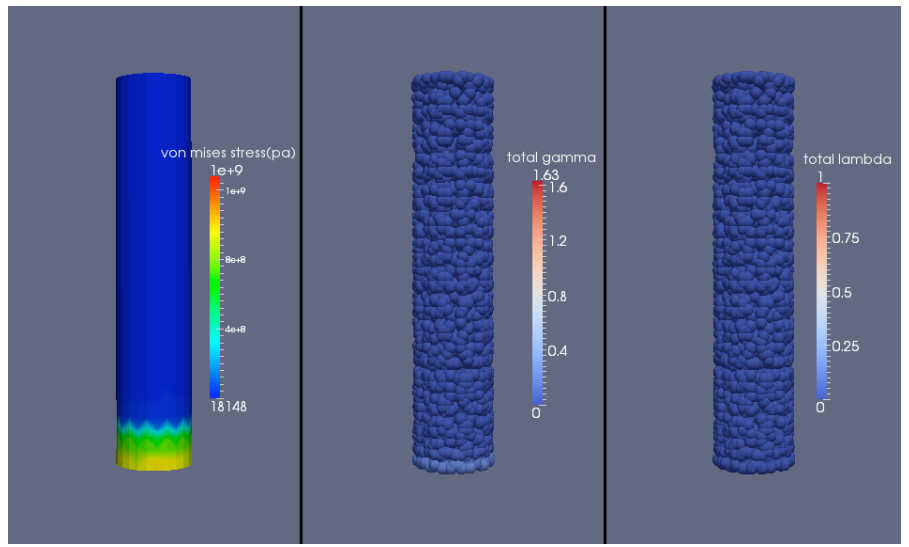

Figure 6.10: Taylor anvil impact simulation of an iron bar for $\alpha-\varepsilon$ transformation at $227 \mathrm{~m} / \mathrm{s}$. (Step 2.) The left field shows the von Mises stress; the center field shows the accumlated slip, summed over all systems; the right field shows the total martensite volume fraction, summed over all variants. We see the elastic precursor travelling upwards, and initiation of plasticity at the base of the specimen.

We note that, with the additional non-negative constraints on the change in volume fraction, we could directly make use of the improved projected-Newton or SQP algorithms described in [66] for optimizing the active constraint sets; this would be advantageous as the $\alpha-\varepsilon$ model seems to suffer more from the oscillations associated with the basic algorithm.

\subsection{Taylor bar: $\alpha-\varepsilon$}

We again simulate a Taylor impact experiment for a $32.4 \mathrm{~mm}$ cyclinder with $3.2 \mathrm{~mm}$ radius at $227 \mathrm{~m} / \mathrm{s}$, this time using the $\alpha-\varepsilon$ model with 6,616 randomly oriented grains, and the material parameters listed in Section refsec:hexaferrum. We see a similar progression as before, with an elastic precursor followed by an inelastic wave (see Figures 6.10-6.12). However, we note that the inelastic wave in this case is entirely plastic, with no transformation 


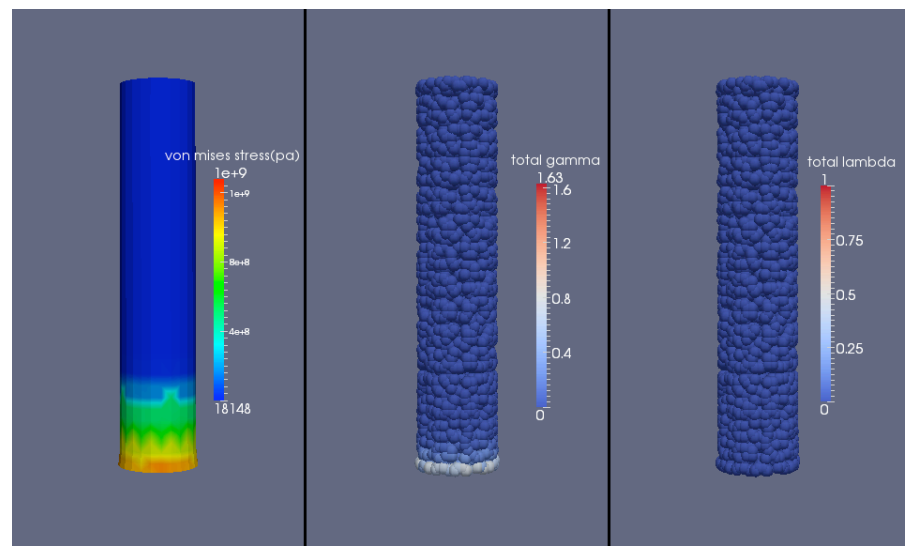

Figure 6.11: Taylor impact simulation of an iron bar for $\alpha-\varepsilon$ transformation at $227 \mathrm{~m} / \mathrm{s}$. (Step 3.) We see the onset of a plastic wave, propagating upwards.)

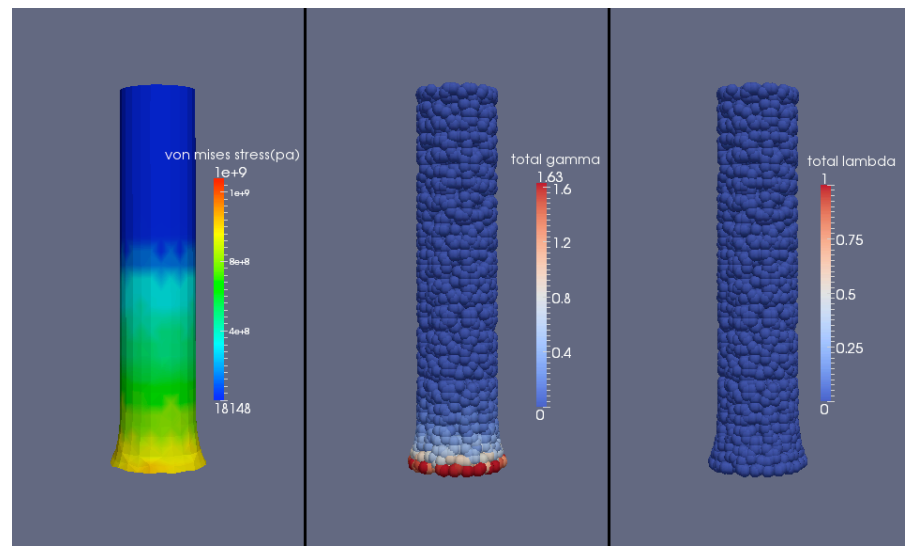

Figure 6.12: Taylor impact simulation of an iron bar for $\alpha-\varepsilon$ transformation at $227 \mathrm{~m} / \mathrm{s}$. (Step 7.) We see the plastic wave continue, and note that no transformation or twinning is observed. 
or twinning observed.

Evidently, for the material parameters used, a very high velocity $(>227 \mathrm{~m} / \mathrm{s})$ is needed to trigger the $\alpha-\varepsilon$ transformation. Additional simulations were started for $1000 \mathrm{~m} / \mathrm{s}$, then $500 \mathrm{~m} / \mathrm{s}$. However, the temperature rise associated with these velocities quickly approached (and surpassed) the melt temperature of iron, apparently exceeding the limitations of the linear-thermal constitutive laws used in this implementation. (See Figure 6.13.)

\subsection{Split-Hopkinson Pressure Bar}

A Split-Hopkinson Pressure Bar (SHPB), also called a Kolsky bar, is another commonly used experimental technique to characterize materials at moderately high strain rates [39]. The traditional method is used for determining the compressive stress-strain response of a material subjected to uniaxial (1-D) plane strain. Modern setups consist of a striker bar, an incident bar, an output bar, and a relatively short (thin) specimen sandwiched between the later two. Initially, the incident bar, specimen, and output bar are aligned coaxially (end-to-end-to-end) in static contact. The striker bar is accelerated (typically using a gas gun) and caused to impact the incident bar at some given velocity. This impact creates a coaxial compressive wave in both bars (travelling in opposite directions). In the striker bar, this wave travels back to the free end of the bar, and reflects as a tension wave; when this tension wave again encounters the interface with the incident bar, the two separate, such that the compression pulse in the incident bar is twice the 'length' of the incident bar. 


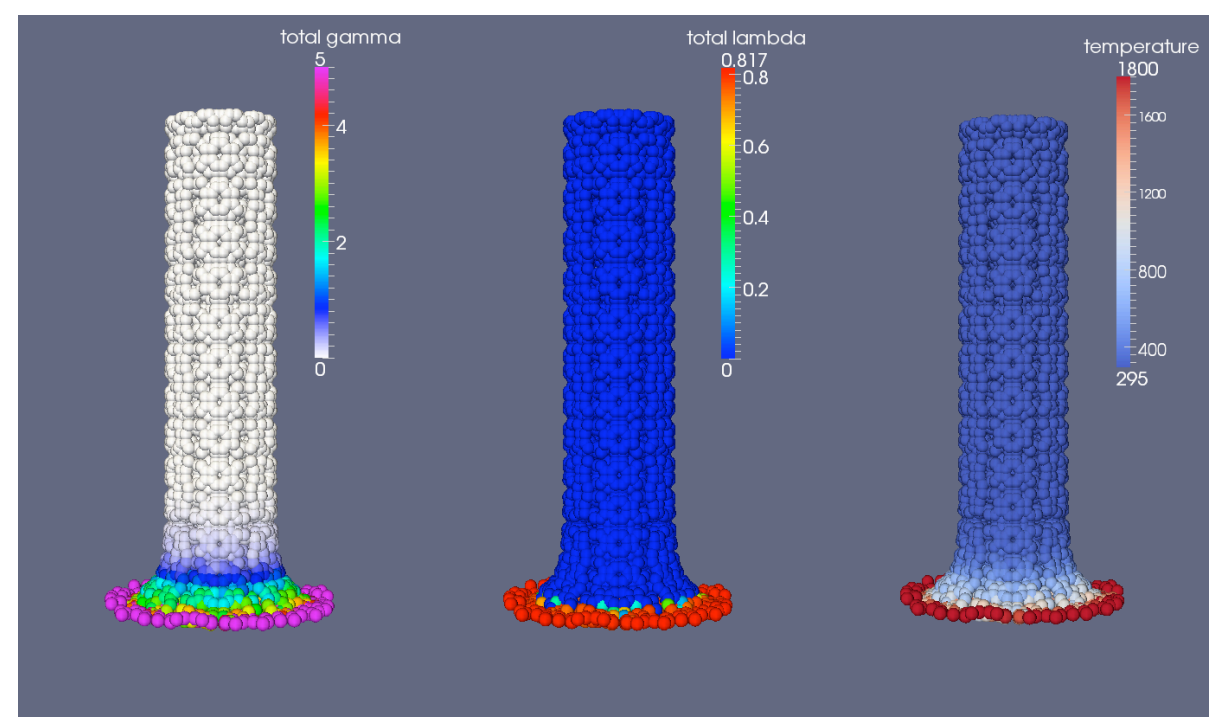

(a)

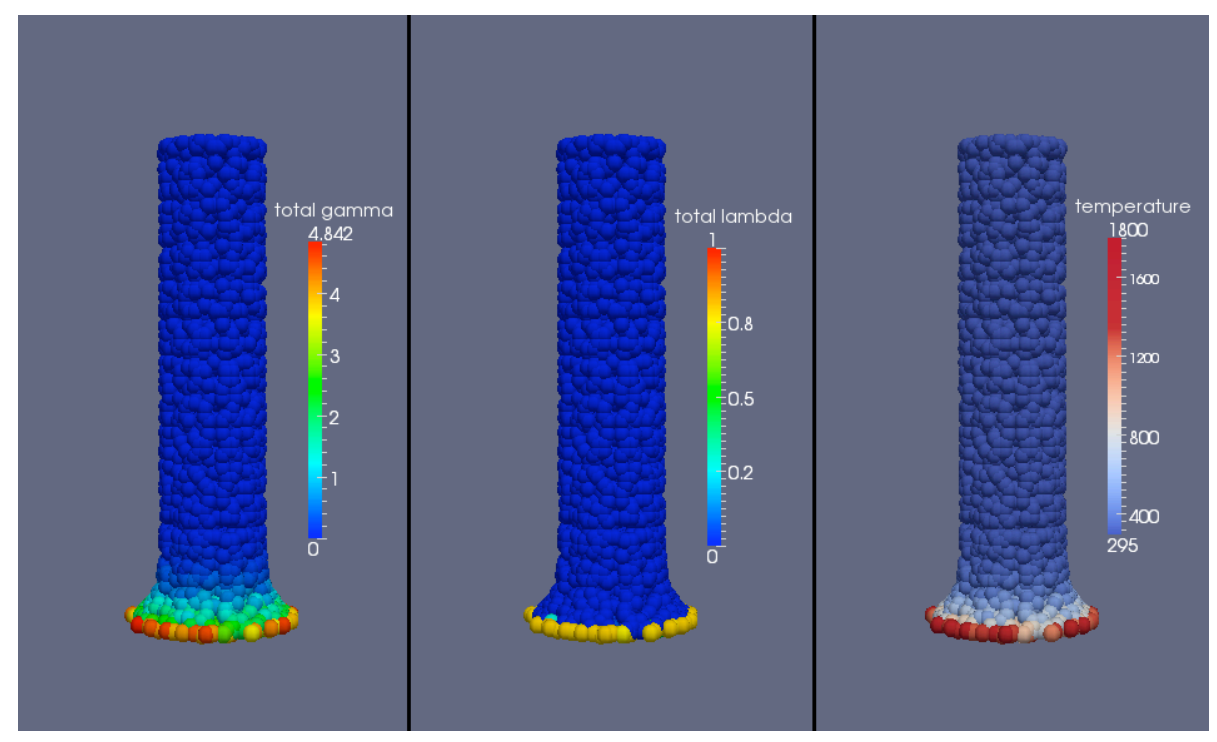

(b)

Figure 6.13: Simulated Taylor bar for $\alpha-\varepsilon$ transformation in iron at (a) $1000 \mathrm{~m} / \mathrm{s}$, and (b) $500 \mathrm{~m} / \mathrm{s}$, showing "melt" before transformation. 


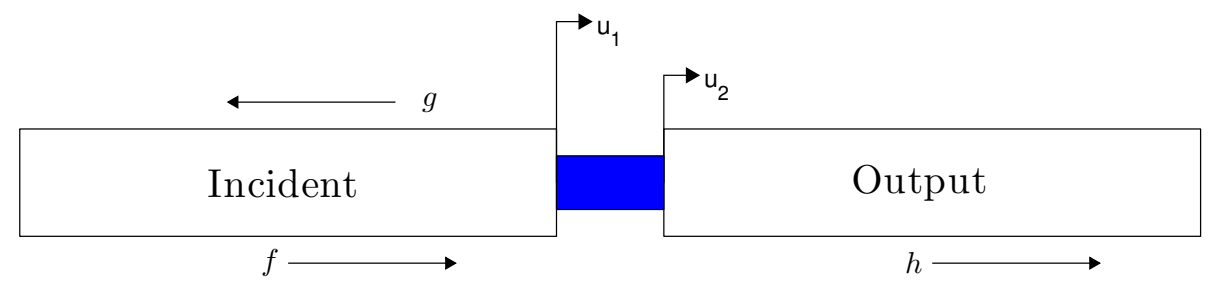

Figure 6.14: Schematic for Split-Hopkinson Pressure Bar.

Meanwhile, the compression pulse in the incident bar, which we denote by $f(x)$, travels along the length of the bar (where it can be measured using strain gages) until it encounters the interface with the specimen. At the interface, a portion of the wave is reflected back into the incident bar, which we denote by $g(x)$, and a portion is transmitted through the specimen to the output bar, denoted by $h(x)$. It is assumed that, since the specimen is short relative to the length of the compression pulse, the specimen is in static equilibrium at every moment. Ideally, the incident and output bars are assumed to have a perfectly linear-elastic response during the test, with no dispersion or dissipation of the waves.

Following the development of [10] and [80], the particle displacement in the bar is merely the superposition of the incident and reflected waves,

$$
u_{1}(x, t)=f(x-c t)+g(x+c t)
$$


such that the particle velocity, strain, and stress in the incident bar can be computed by

$$
\begin{aligned}
& v_{1}(x, t)=\frac{\partial u_{1}}{\partial t}(x, t)=c\left[-f^{\prime}(x-c t)+g^{\prime}(x+c t)\right] \\
& \varepsilon_{1}(x, t)=\frac{\partial u_{1}}{\partial x}(x, t)=f^{\prime}(x-c t)+g^{\prime}(x+c t) \\
& \sigma_{1}(x, t)=E_{1} \varepsilon_{1}(x, t)=E_{1}\left[f^{\prime}(x-c t)+g^{\prime}(x+c t)\right]
\end{aligned}
$$

In the output bar, we have only the transmitted wave,

$$
u_{2}(x, t)=h(x-c t)
$$

from which we can compute the analogous quantities,

$$
\begin{aligned}
& v_{2}(x, t)=\frac{\partial u_{2}}{\partial t}(x, t)=-c h^{\prime}(x-c t) \\
& \varepsilon_{2}(x, t)=\frac{\partial u_{2}}{\partial x}(x, t)=h^{\prime}(x-c t) \\
& \sigma_{2}(x, t)=E_{2} \varepsilon_{2}(x, t)=E_{2} h^{\prime}(x-c t)
\end{aligned}
$$

In the specimen, we use the engineering approximation for the strain and strain rate, 
s.t.

$$
\begin{aligned}
& \varepsilon(t)=\frac{u_{2}(0, t)-u_{1}(0, t)}{L} \\
& \dot{\varepsilon}(t)=\frac{v_{2}(0, t)-v_{1}(0, t)}{L}
\end{aligned}
$$

Since the specimen is small, we assume the axial stress in the specimen is uniform at every instant, whereby equilibrium gives us,

$$
\sigma(t) A=\sigma_{1}(0, t) A_{1}=\sigma_{2}(0, t) A_{2}
$$

By substitution we find,

$$
\begin{aligned}
\dot{\varepsilon}(t) & =\frac{c\left[-h^{\prime}(-c t)+f^{\prime}(-c t)-g^{\prime}(c t)\right]}{L} \\
\sigma(t) & =\frac{E_{1} A_{1}}{A}\left[f^{\prime}(-c t)+g^{\prime}(c t)\right]=\frac{E_{2} A_{2}}{A} h^{\prime}(-c t)
\end{aligned}
$$

Assuming the incident and output bars have the same mechanical properties,

$$
E_{1}=E_{2} \quad \& \quad A_{1}=A_{2} \quad \Longrightarrow \quad f^{\prime}(-c t)+g^{\prime}(c t)=h^{\prime}(-c t)
$$

we simplify the the above equations to find that the state of the specimen can be computed 


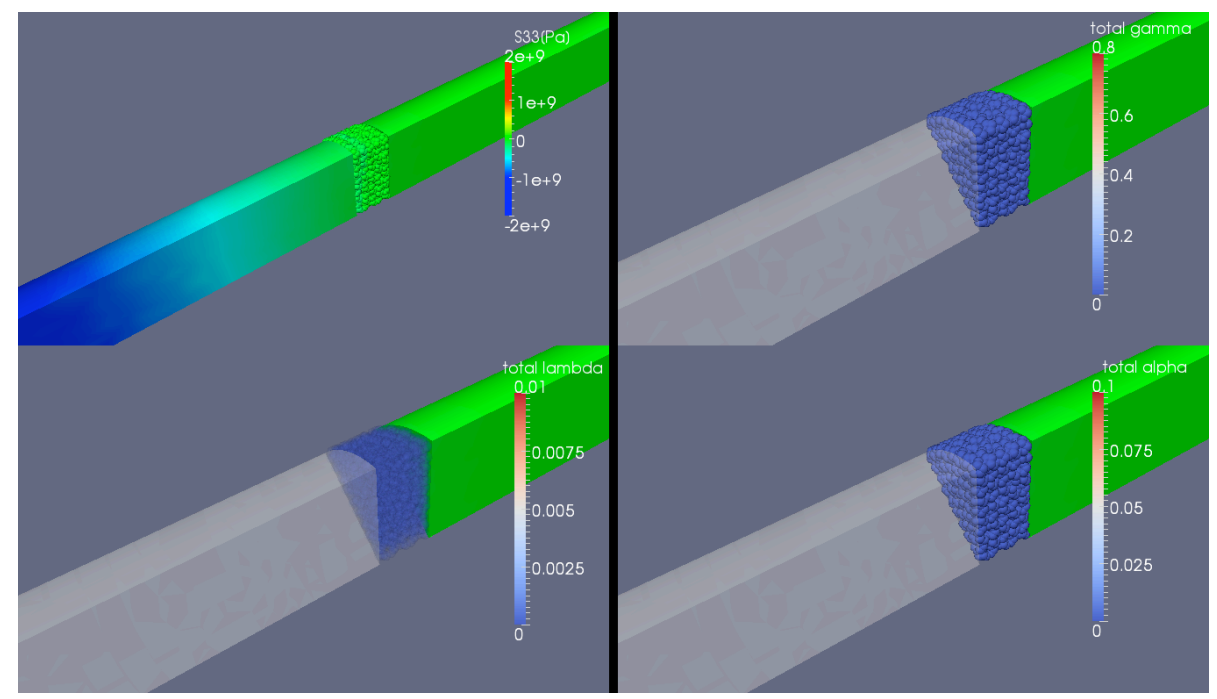

Figure 6.15: SHPB simulation of an iron specimen for $\alpha-\varepsilon$ transformation at $50 \mathrm{~m} / \mathrm{s}$. (Step 15.) The fields depict a cut-away view along an axisymmetric cylinder. The top left field shows the axis stress; the top right field shows the accumlated slip, summed over all systems; the bottom left field shows the total martensite volume fraction, summed over all variants; the bottom right field shows the total austenite twin volume fraction, summed over all variants. The incident and output bars are depicted as solid, and colormapped by the axial stress in each of the panels. The specimen is depicted by spherical glyphs at the material points. The incoming compression pulse travels from lower-left to upper right in the figure.

from the traces of just the incident and output waveforms,

$$
\begin{aligned}
\dot{\varepsilon}(t) & =\frac{2 c\left[f^{\prime}(-c t)-h^{\prime}(-c t)\right]}{L} \\
\sigma(t) & =\frac{E_{b} A_{b}}{A} h^{\prime}(-c t)
\end{aligned}
$$

We now investigate the response of our model at four (5) different impact velocities rates in a simulated SHPB experiment: $10 \mathrm{~m} / \mathrm{s}, 50 \mathrm{~m} / \mathrm{s}, 100 \mathrm{~m} / \mathrm{s}, 200 \mathrm{~m} / \mathrm{s}$, and $1000 \mathrm{~m} / \mathrm{s}$. For the simulation, we construct an axisymmetric (perfectly-bonded) composite rod, where 


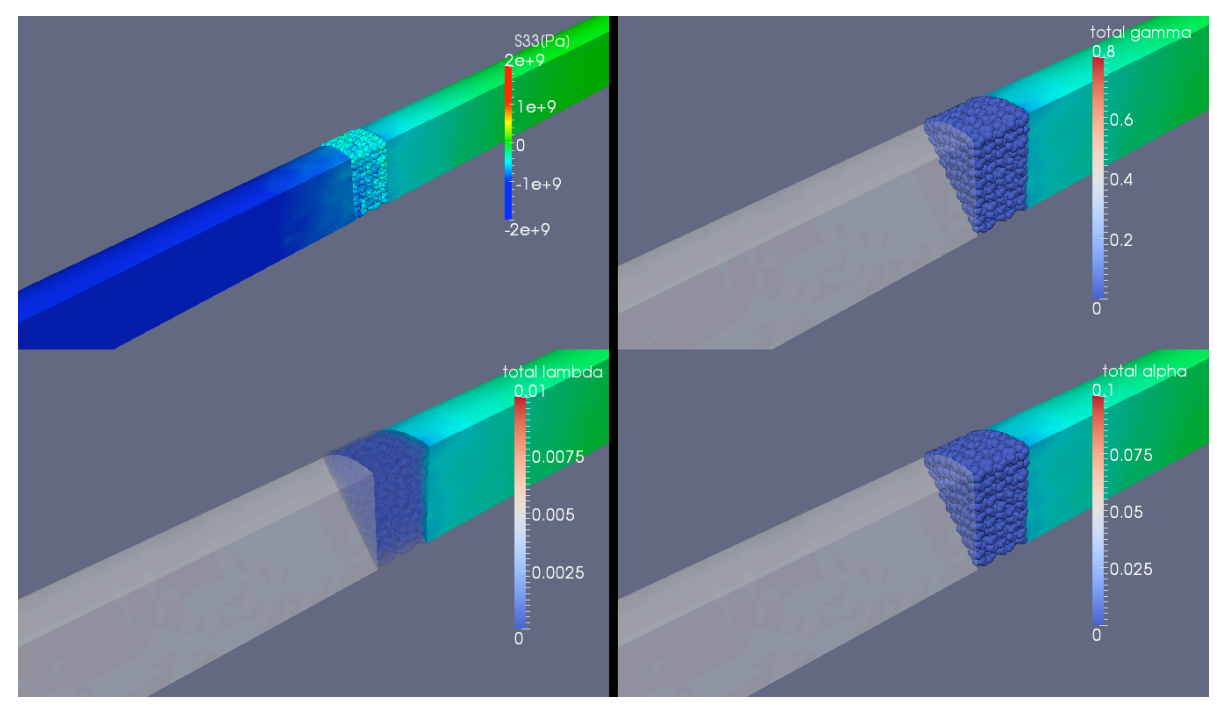

Figure 6.16: SHPB simulation of an iron specimen for $\alpha-\varepsilon$ transformation at $50 \mathrm{~m} / \mathrm{s}$. (Step 20.) The pulse reaches the specimen, which deforms elastically.

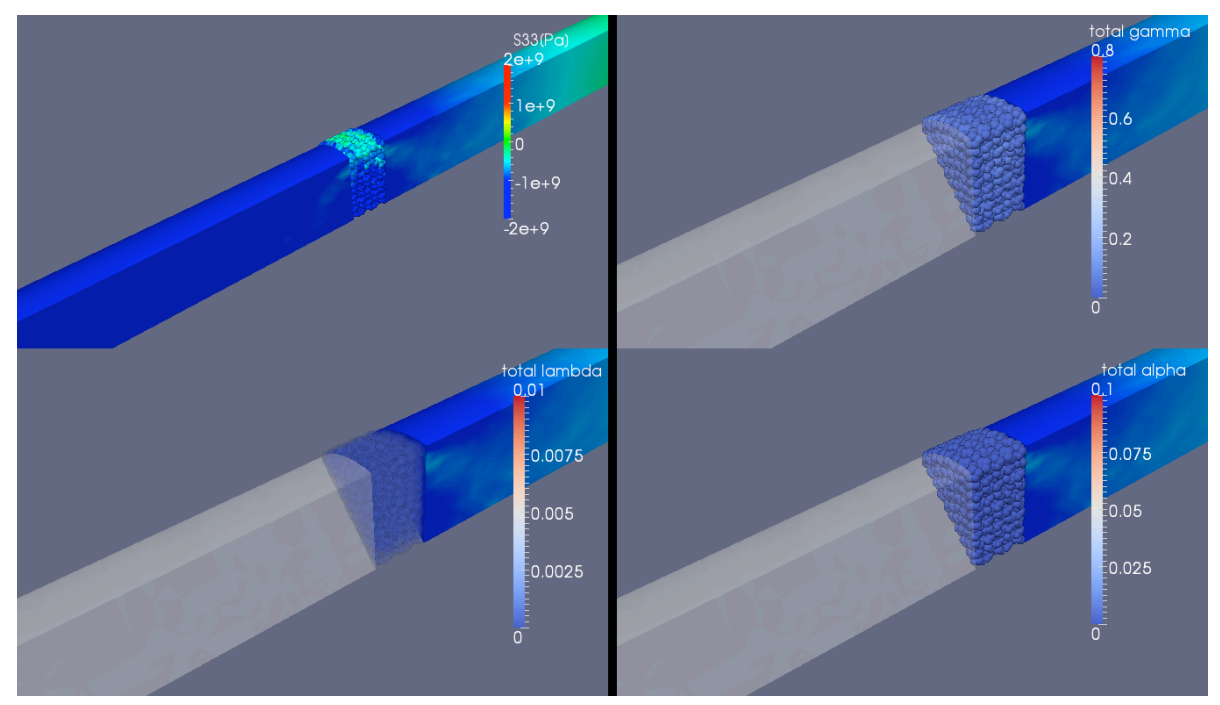

Figure 6.17: SHPB simulation of an iron specimen for $\alpha-\varepsilon$ transformation at $50 \mathrm{~m} / \mathrm{s}$. (Step 25.) The pressure pulse continues, and stresses can be seen localizing along the top of the specimen as radial deformations become significant. The specimen response is still elastic, however. 


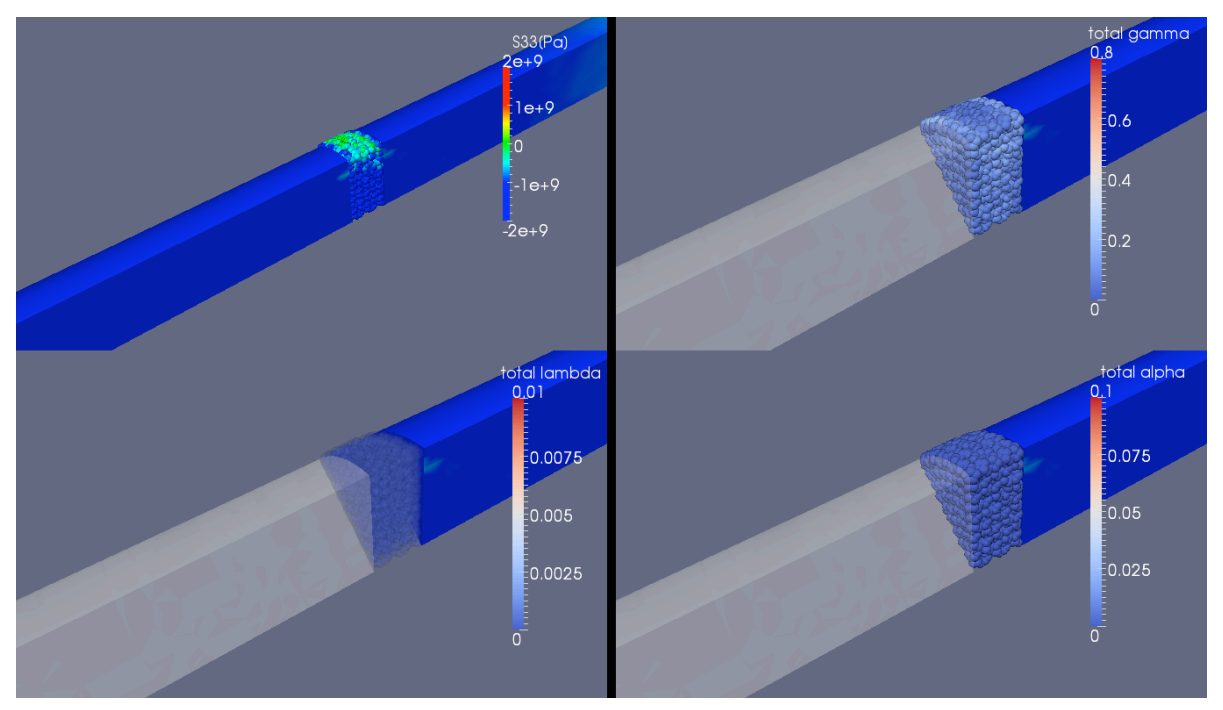

Figure 6.18: SHPB simulation of an iron specimen for $\alpha-\varepsilon$ transformation at $50 \mathrm{~m} / \mathrm{s}$. (Step 30.) Plasticity is seen to initiate along interior planes in the specimen.

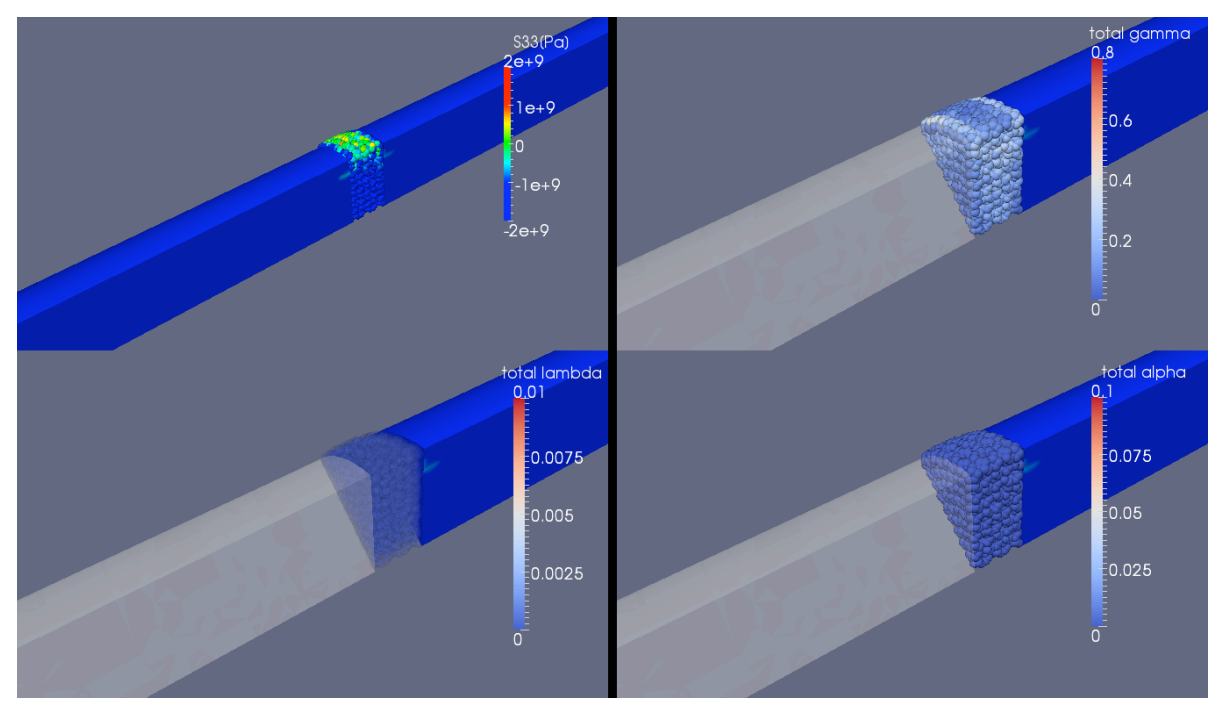

Figure 6.19: SHPB simulation of an iron specimen for $\alpha-\varepsilon$ transformation at $50 \mathrm{~m} / \mathrm{s}$. (Step 35.) Bands of plasticity begin to develop. 


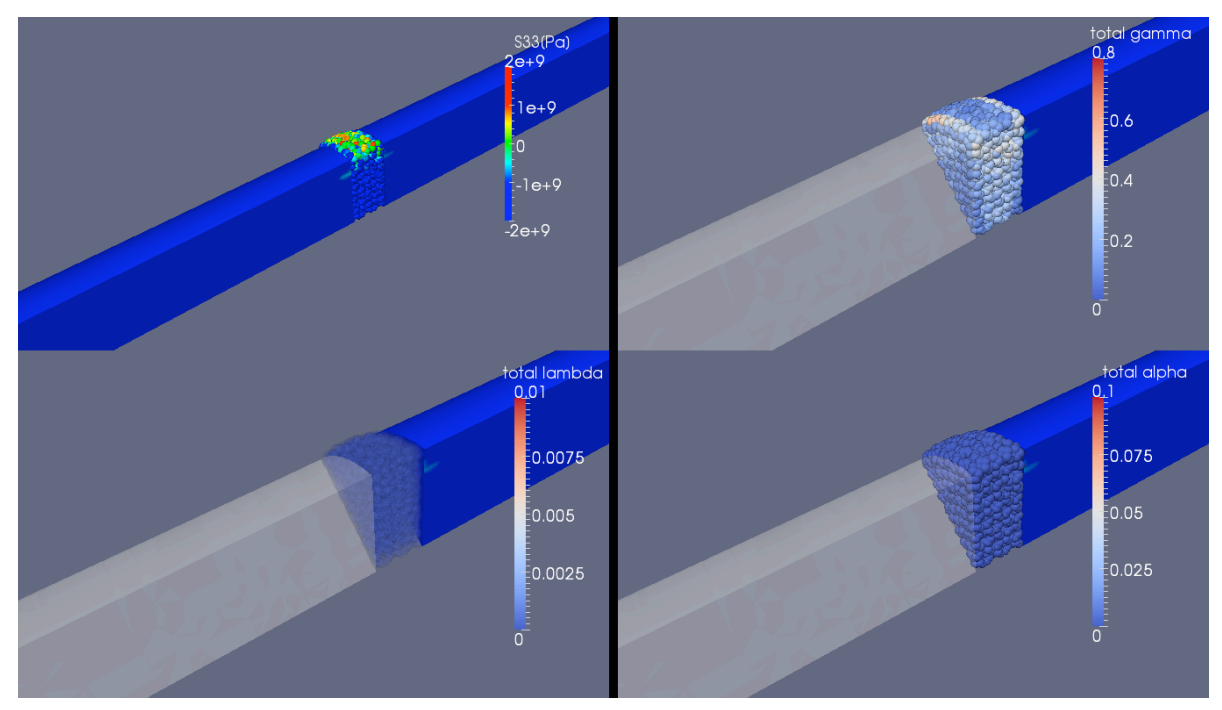

Figure 6.20: SHPB simulation of an iron specimen for $\alpha-\varepsilon$ transformation at $50 \mathrm{~m} / \mathrm{s}$. (Step 40.) Bands of plasticity continue to develop.

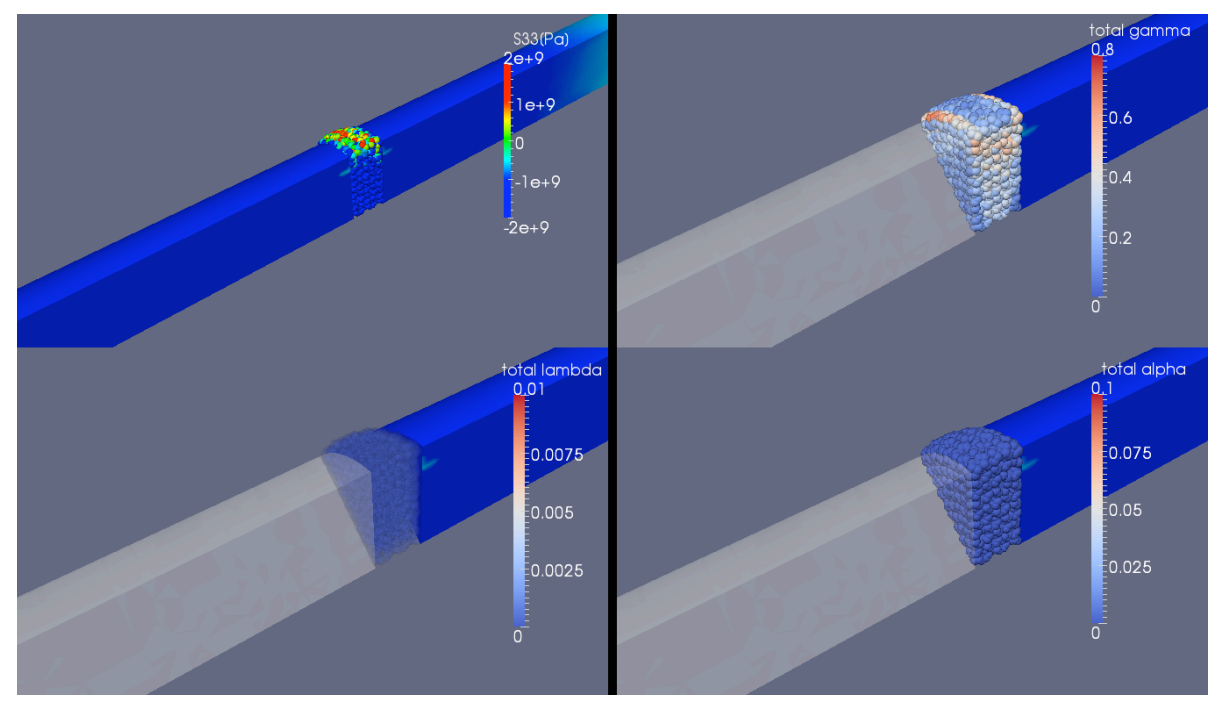

Figure 6.21: SHPB simulation of an iron specimen for $\alpha-\varepsilon$ transformation at $50 \mathrm{~m} / \mathrm{s}$. (Step 45.) Bands of plasticity continue to develop. 


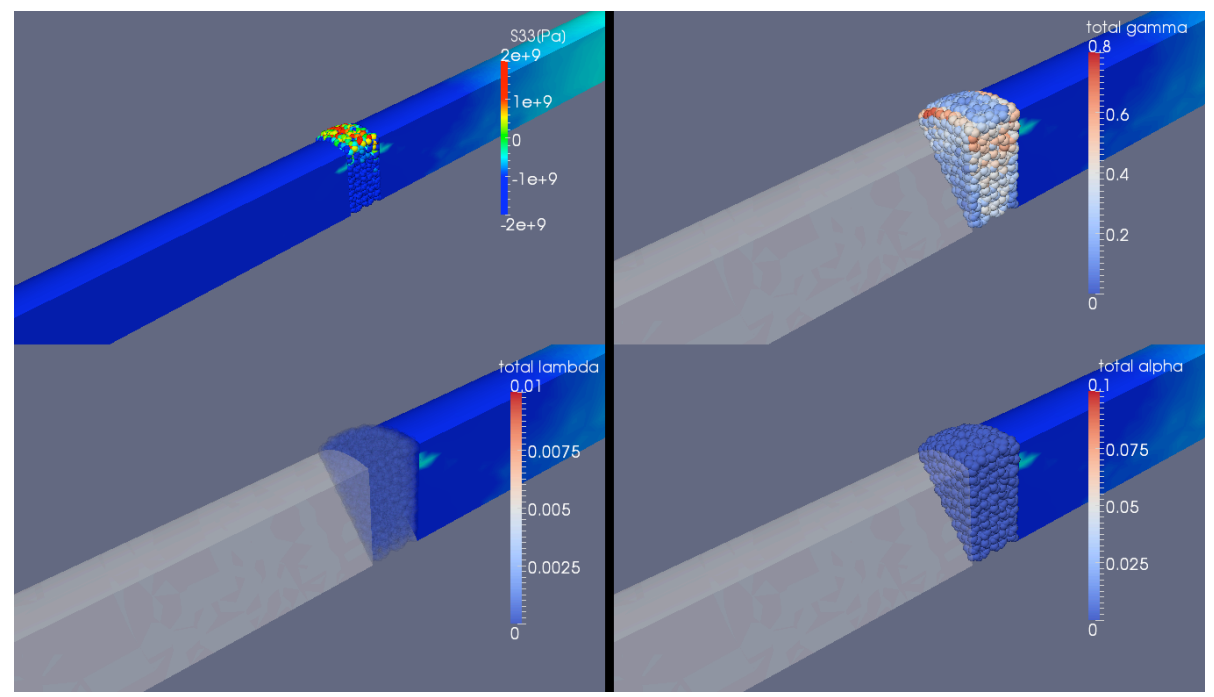

Figure 6.22: SHPB simulation of an iron specimen for $\alpha-\varepsilon$ transformation at $50 \mathrm{~m} / \mathrm{s}$. (Step 50.) An elastic tensile pulse reflected from the free end of the output bar can be seen in the upper right corner, travelling back down bar from top-right to bottom left.

the ends are prescribed a linear-elastic material model consistent with isotropic steel, and the center portion is assigned our framework for transformation, twinning, and plasticity. The simulated incident and output bars measure $25.0 \mathrm{~mm}$ in length with a $6.0 \mathrm{~mm}$ radius; the center specimen region measures $1.5 \mathrm{~mm}$. We use 1,849 randomly oriented grains in the center region to simulate the specimen.

In our simulation, the velocity pulse is effectively longer than the input and output bars, so a release wave from the free-end of the output bar reflects back to the specimen before the pulse terminates. The peak stress in the elastic bars can be predicted from 1-D theory for weak shocks, s.t.

$$
\sigma=\rho_{0} c v \approx 8000 \frac{\mathrm{kg}}{\mathrm{m}^{3}} \cdot 5000 \frac{\mathrm{m}}{\mathrm{s}} \cdot v=40 v \frac{\mathrm{MPa}}{\mathrm{m} / \mathrm{s}}
$$




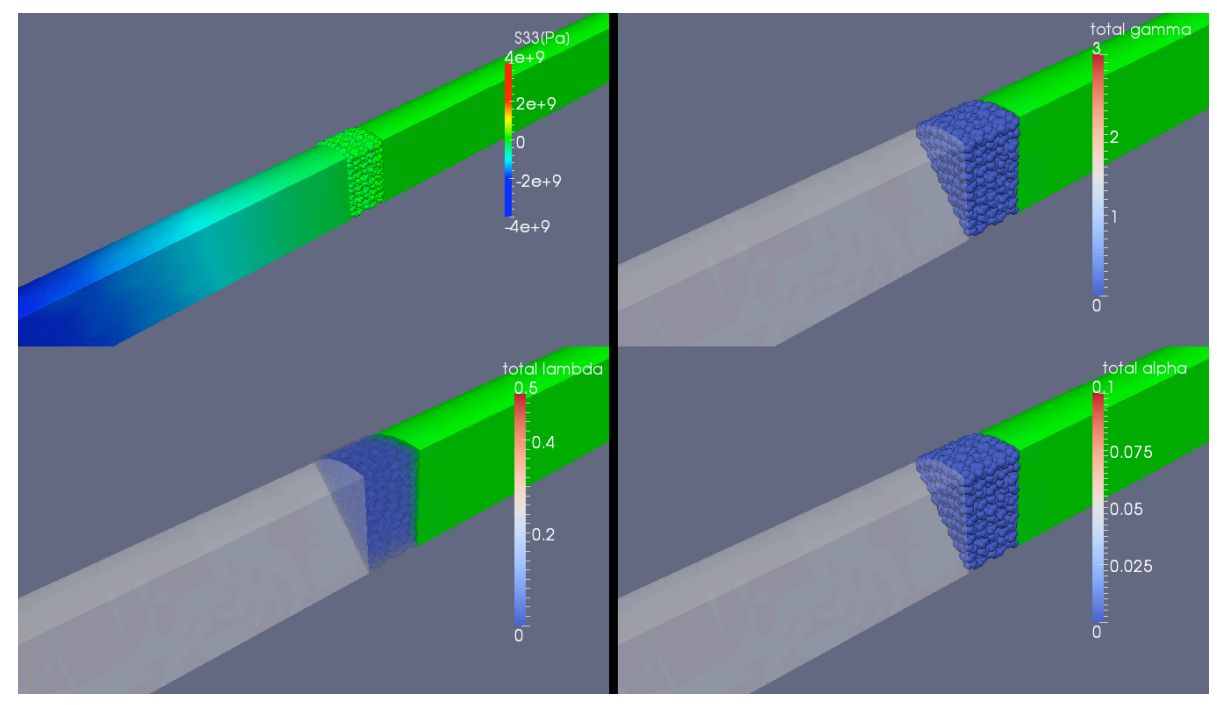

Figure 6.23: SHPB simulation of an iron specimen for $\alpha-\varepsilon$ transformation at $100 \mathrm{~m} / \mathrm{s}$. (Step 15.) The fields depict a cut-away view along an axisymmetric cylinder. The top left field shows the axis stress; the top right field shows the accumlated slip, summed over all systems; the bottom left field shows the total martensite volume fraction, summed over all variants; the bottom right field shows the total austenite twin volume fraction, summed over all variants. The incident and output bars are depicted as solid, and colormapped by the axial stress in each of the panels. The specimen is depicted by spherical glyphs at the material points. The incoming compression pulse travels from lower-left to upper right in the figure. 


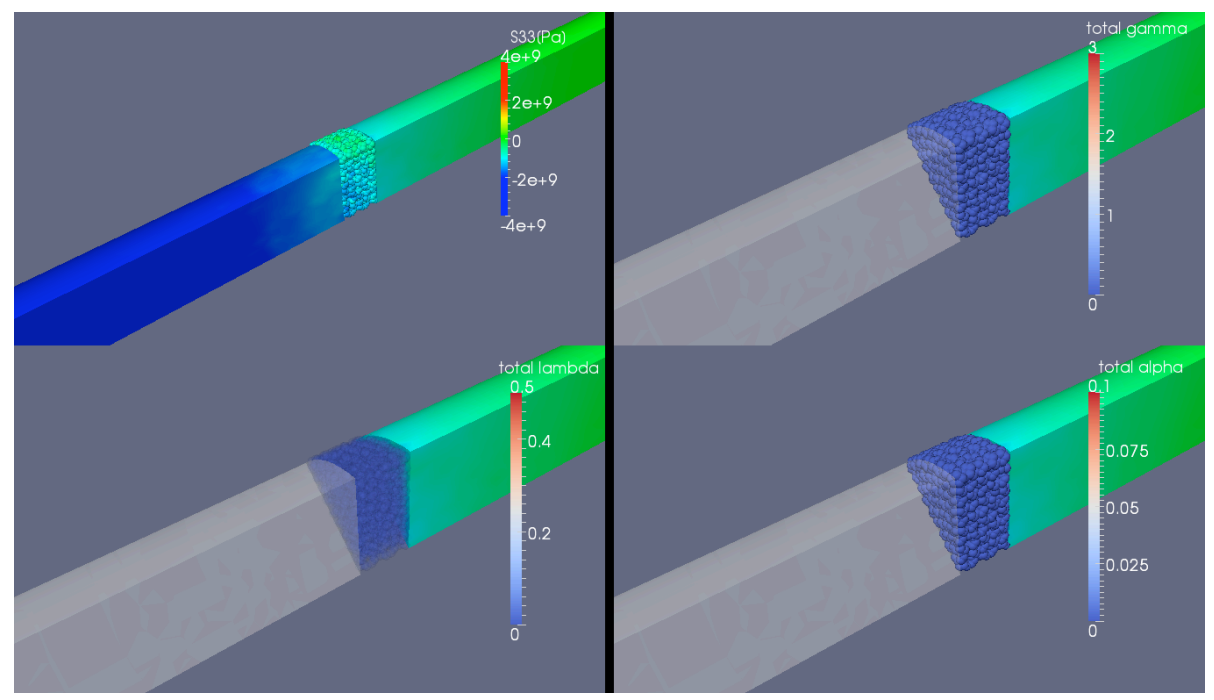

Figure 6.24: SHPB simulation of an iron specimen for $\alpha-\varepsilon$ transformation at $100 \mathrm{~m} / \mathrm{s}$. (Step 20.) The pulse reaches the specimen, which deforms elastically.

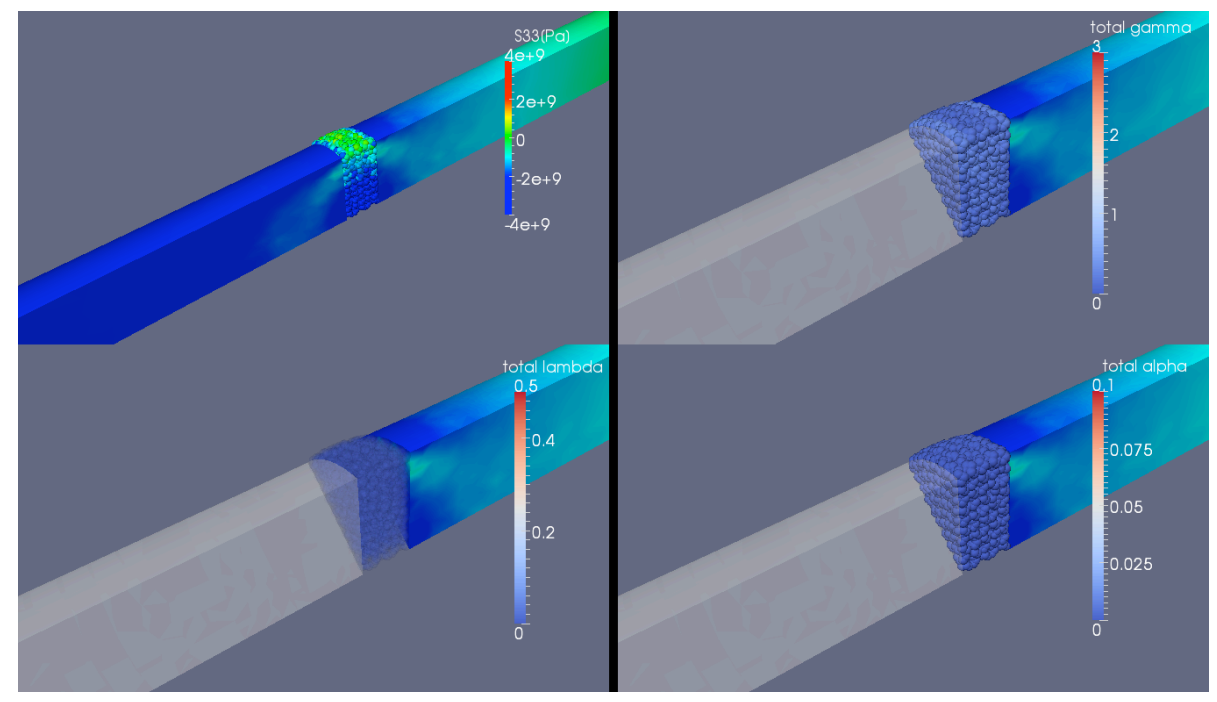

Figure 6.25: SHPB simulation of an iron specimen for $\alpha-\varepsilon$ transformation at $100 \mathrm{~m} / \mathrm{s}$. (Step 25.) The pressure pulse continues, and stresses can be seen localizing along the top of the specimen as radial deformations become significant. Stress lobes near the specimen interface are evident in both the specimen and the bar. The specimen response is still mostly elastic, however. 


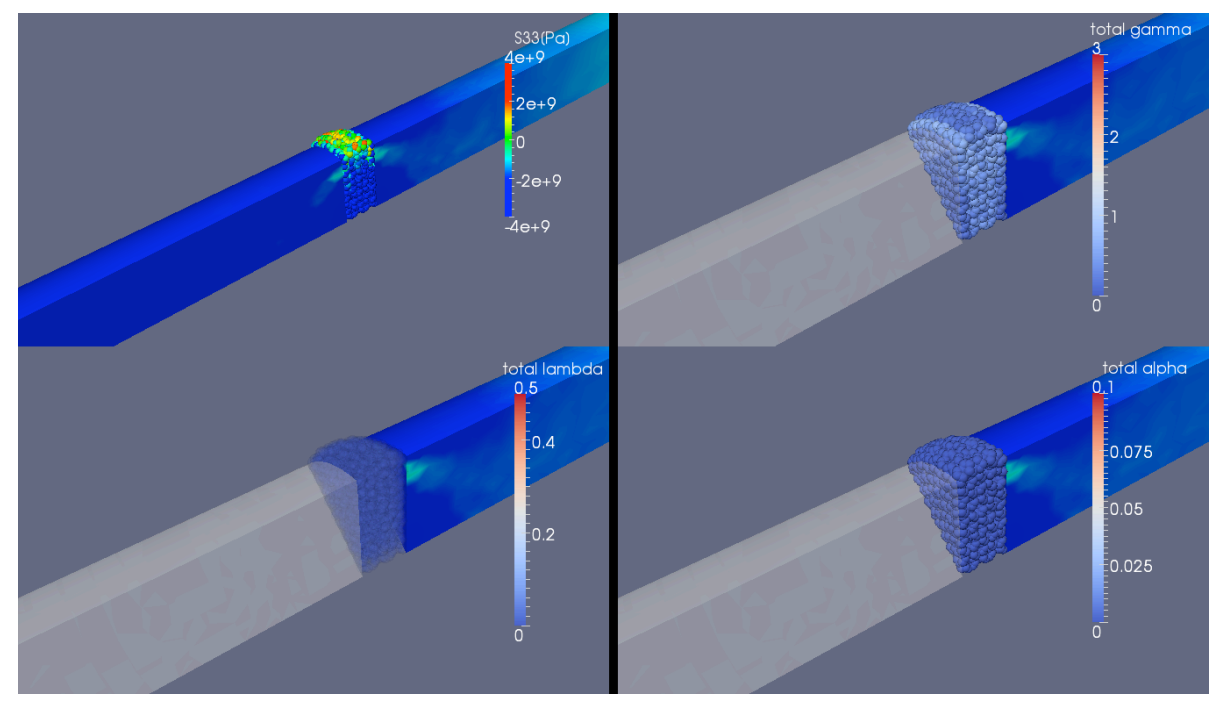

Figure 6.26: SHPB simulation of an iron specimen for $\alpha-\varepsilon$ transformation at $100 \mathrm{~m} / \mathrm{s}$. (Step 30.) A "wedge" of plasticity is seen to initiate along interior planes in the specimen, but still no transformation.

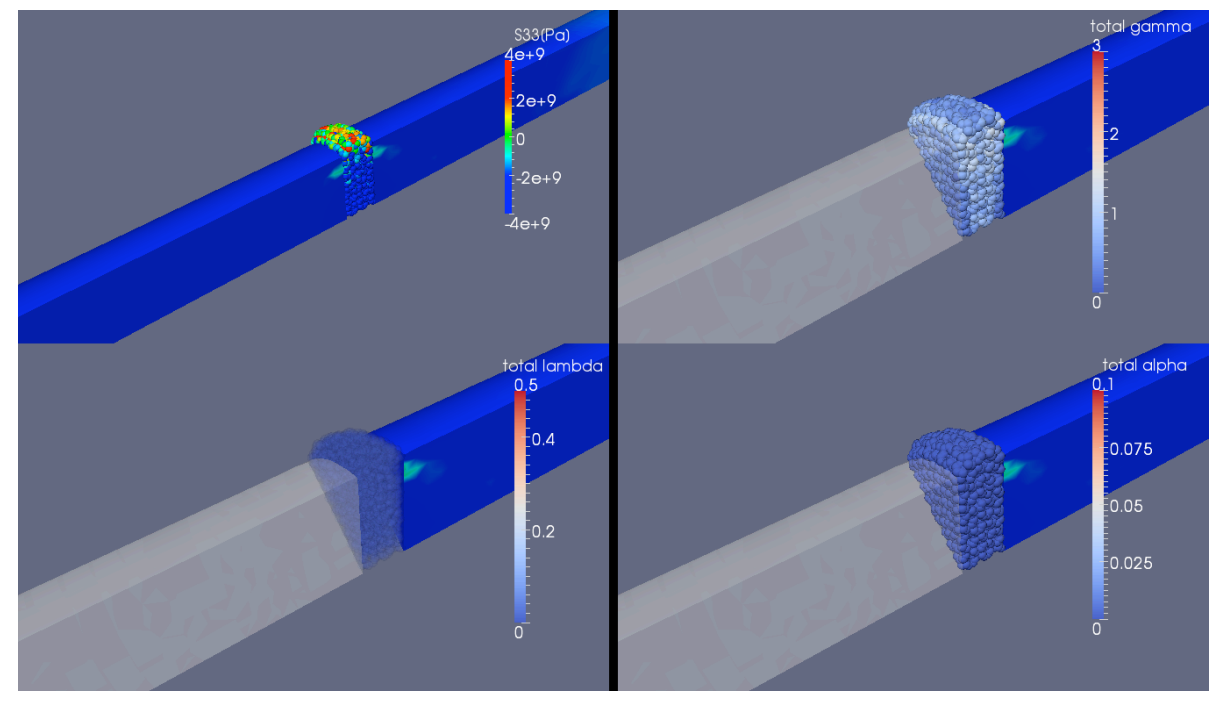

Figure 6.27: SHPB simulation of an iron specimen for $\alpha-\varepsilon$ transformation at $100 \mathrm{~m} / \mathrm{s}$. (Step 35.) Bands of plasticity continue to develop. 


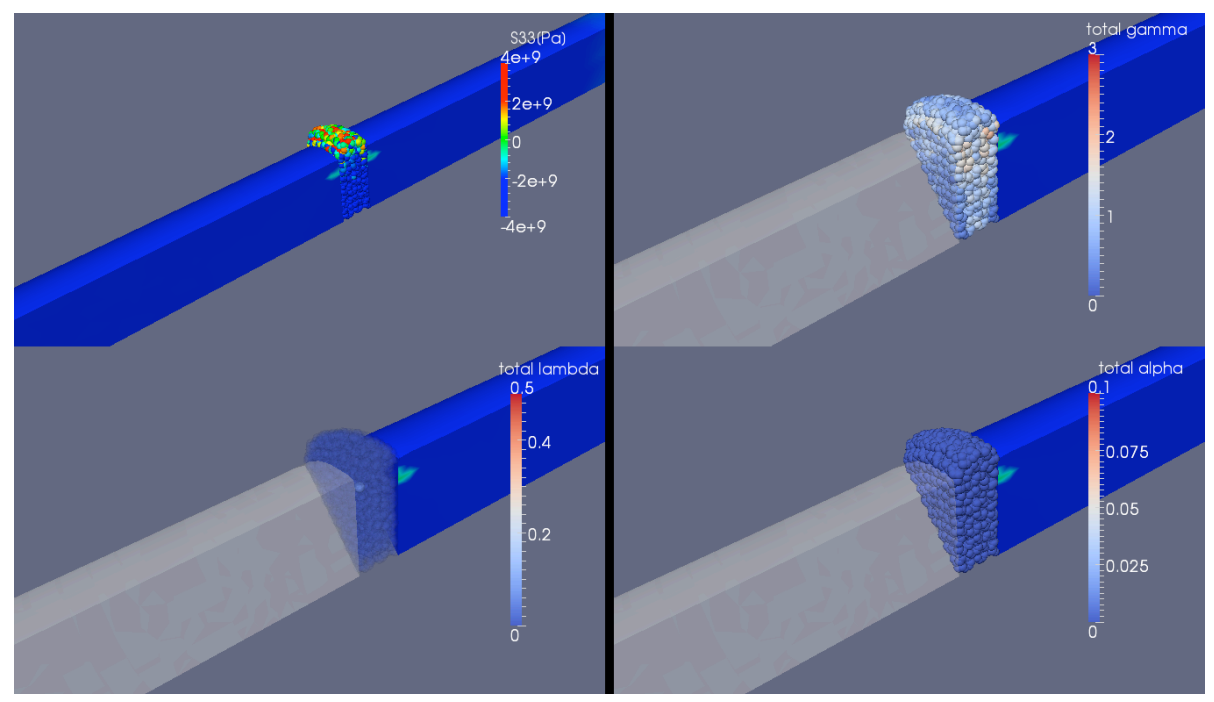

Figure 6.28: SHPB simulation of an iron specimen for $\alpha-\varepsilon$ transformation at $100 \mathrm{~m} / \mathrm{s}$. (Step 40.) Bands of plasticity continue to develop.

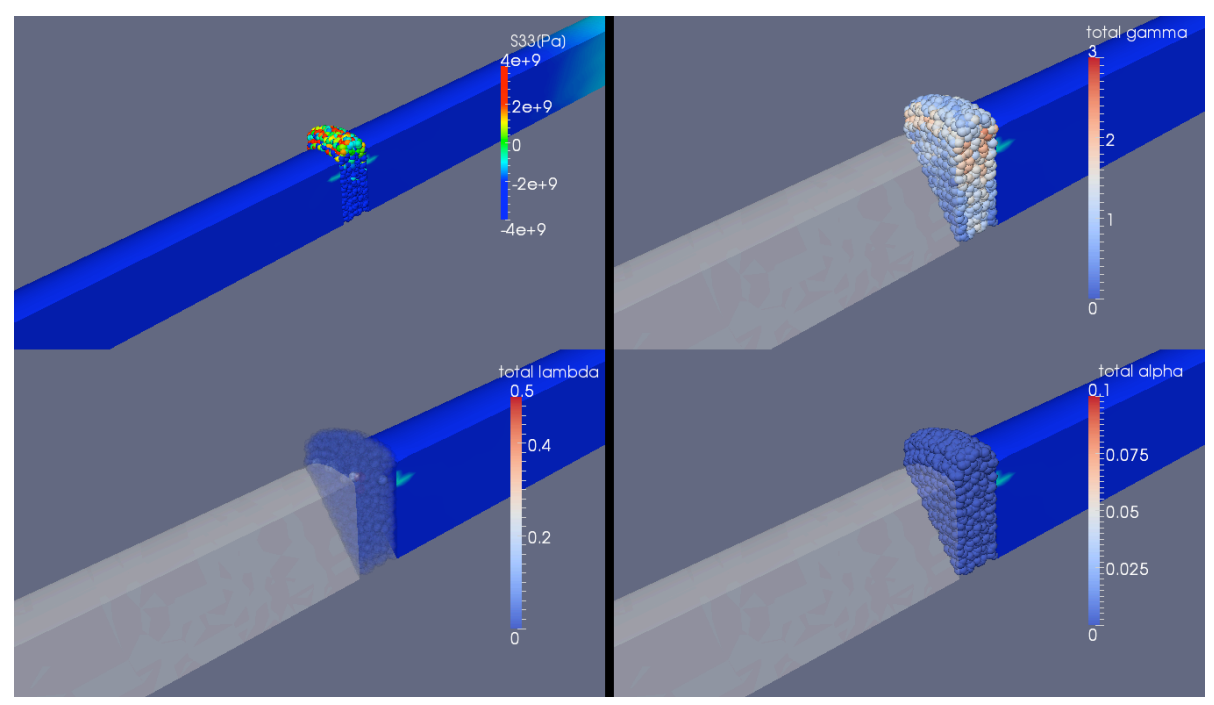

Figure 6.29: SHPB simulation of an iron specimen for $\alpha-\varepsilon$ transformation at $100 \mathrm{~m} / \mathrm{s}$. (Step 45.) An isolated spot of transformation is seen, just below the surface of the specimen, near the edge of the incident bar. 


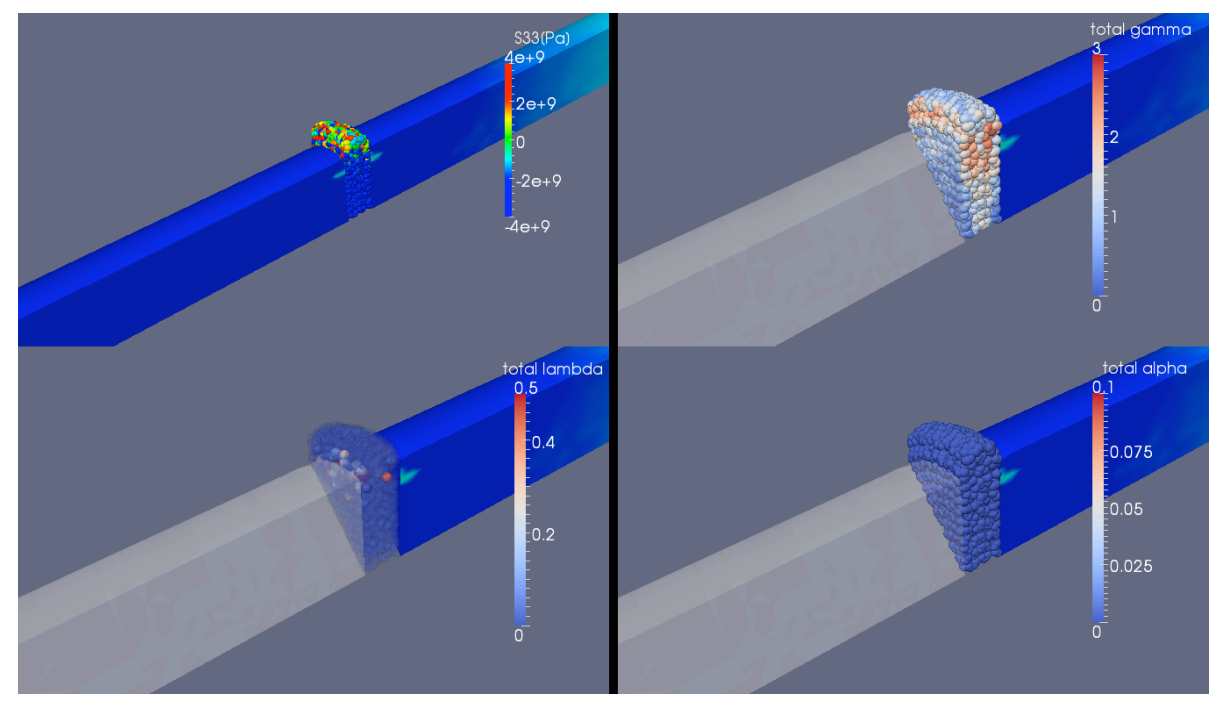

Figure 6.30: SHPB simulation of an iron specimen for $\alpha-\varepsilon$ transformation at $100 \mathrm{~m} / \mathrm{s}$. (Step 50.) An elastic tensile pulse reflected from the free end of the output bar can be seen in the upper right corner, travelling back down bar from top-right to bottom left. More transformation is seen to develop, mostly along the edges of the interfaces with the incident and output bars.

which was observed to be within $2-4 \%$ of the peak $\sigma_{33}$ stress observed in the incident bar.

For simulations at $50 \mathrm{~m} / \mathrm{s}$ and below, we observe no transformation, though we do see plasticity, as shown in Figures 6.15-6.22. We see that the stress becomes the largest near the surface of the specimen, where, due to the axial confinement of the incident and output bars, the material begin to extrude outwards radially. The plasticity is observed to form in very distinctive bands.

For simulations at $100 \mathrm{~m} / \mathrm{s}$ and above, we observe transformation initiate in the interior of the specimen, just behind the first plane of material points; this is similar to the initiation in plasticity, as shown in Figures 6.23-6.30. We also begin to see extreme distortion of the specimen, and the one-dimensional assumption clearly breaks down. At sufficiently 


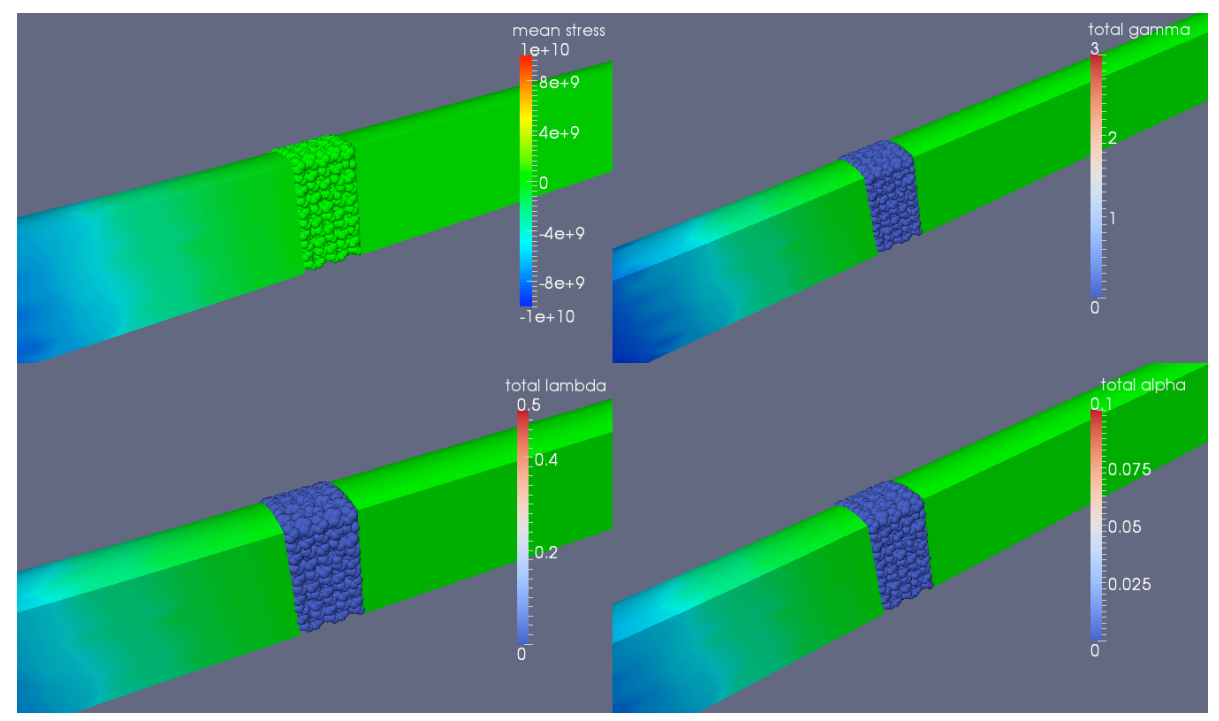

Figure 6.31: SHPB simulation of an iron specimen for $\alpha-\varepsilon$ transformation at $1000 \mathrm{~m} / \mathrm{s}$. (Step 15.) The fields depict a cut-away view along an axisymmetric cylinder. The top left field shows the axis stress; the top right field shows the accumlated slip, summed over all systems; the bottom left field shows the total martensite volume fraction, summed over all variants; the bottom right field shows the total austenite twin volume fraction, summed over all variants. The incident and output bars are depicted as solid, and colormapped by the axial stress in each of the panels. The specimen is depicted by spherical glyphs at the material points. The incoming compression pulse travels from lower-left to upper right in the figure.

(unrealistically) high velocity impacts (e.g. $1000 \mathrm{~m} / \mathrm{s}$; see Figure 6.33), the specimen is completely "pancaked" during the simulation. 


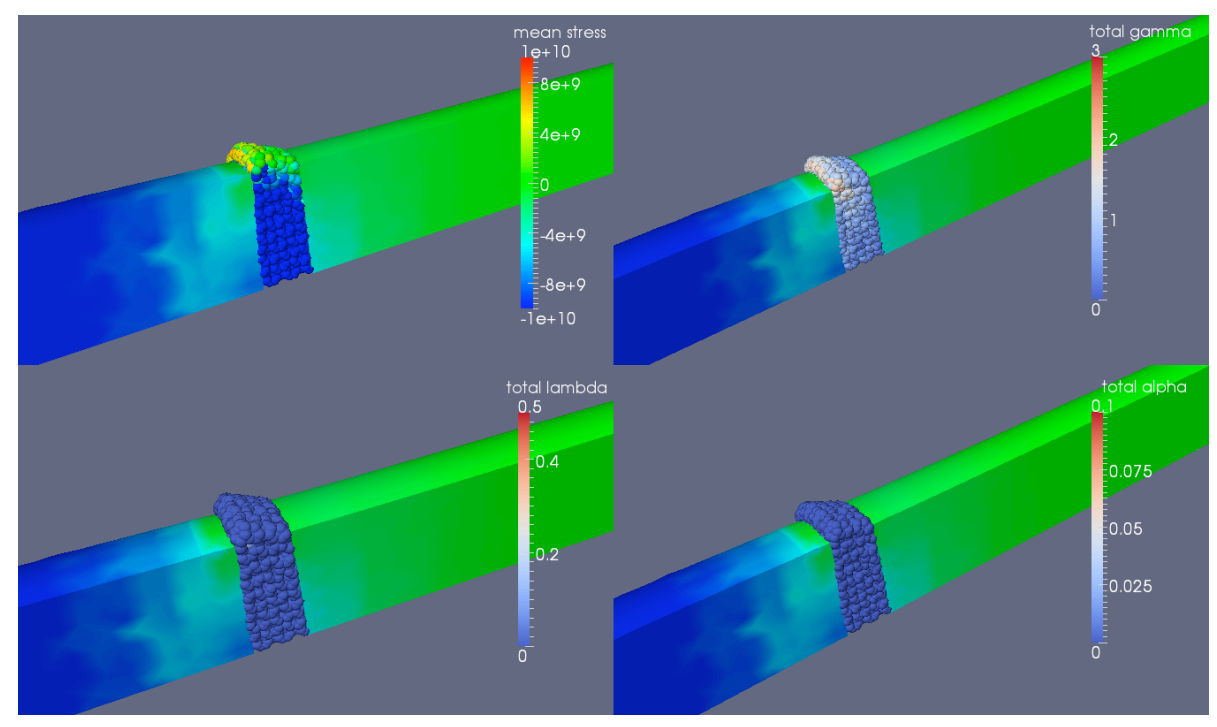

Figure 6.32: SHPB simulation of an iron specimen for $\alpha-\varepsilon$ transformation at $1000 \mathrm{~m} / \mathrm{s}$. (Step 20.) The pulse reaches the specimen, which is already grossly deformed, with significant plasticity. Little transformation is seen.

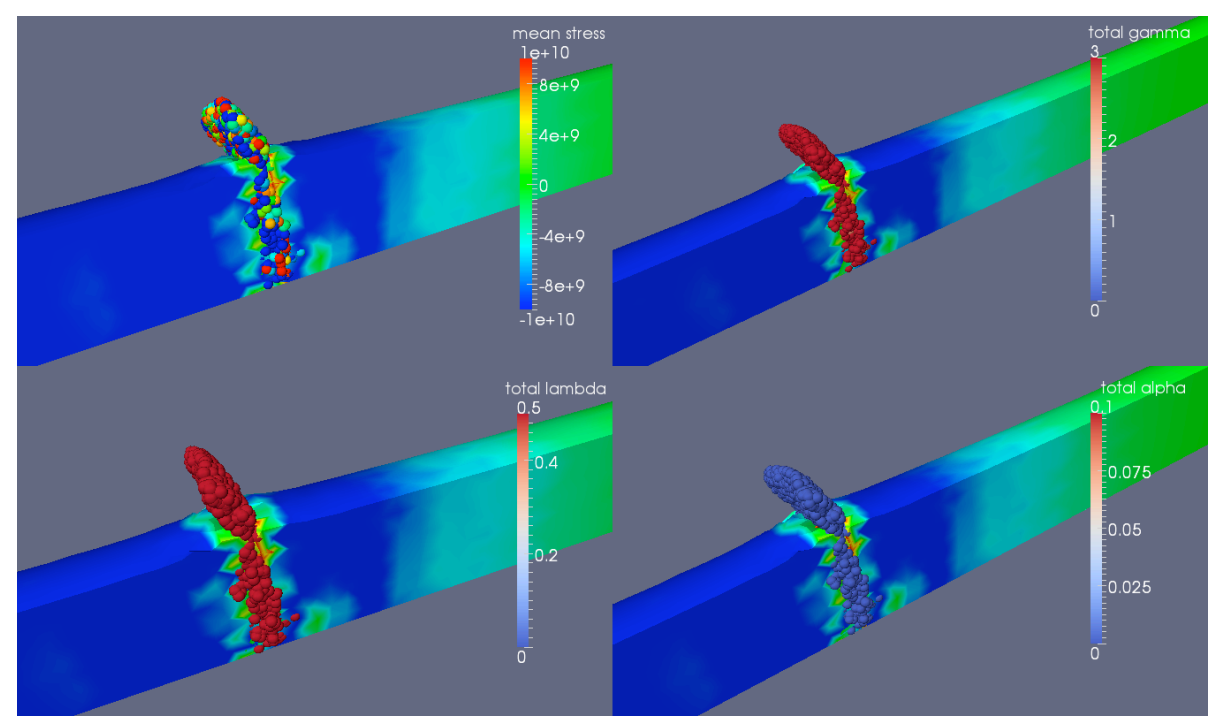

Figure 6.33: SHPB simulation of an iron specimen for $\alpha-\varepsilon$ transformation at $1000 \mathrm{~m} / \mathrm{s}$. (Step 25.) The specimen is destroyed, and the results are clearly non-physical. 


\section{Chapter 7}

\section{Conclusions \& Future Work}

In this thesis, we have recognized a certain similarity between phase transformation and plastic slip, in that both mechanisms serve to reduce the elastic stored energy of a crystal by accommodating deformation along prescribed (constrained) crystallographic directions — essentially competing (or cooperating) constraints on the lattice deformations.

We have proposed a modelling framework to explore the interplay between martensitic phase transformations and plastic slip in polycrystalline materials. We have strived to make the model as simple as possible (but no simpler), with an eye towards computational efficiency. The resulting framework uses a convexified potential for the internal energy density to capture the stored energy associated with transformation at the meso-scale, and introduces kinetic potentials to govern the evolution of transformation and plastic slip. The framework is novel in the way it treats plasticity on par with transformation.

In Chapter 4, in the setting of anti-plane shear, we have presented a computational study of the interplay of stress-induced (iso-thermal) phase transformation and plasticity 
in polycrystalline media. Even in this simple setting, the framework captures a rich array of behaviors associated with SMAs. The following insights are qualitative, but we emphasize features that are generic and expected to hold for the realistic three-dimensional setting.

- Superelasticity. In the situation where the plastic yield strength is very high (infinite), we see that transformation begins in isolated regions when the stress is close to the value of the Sachs constant stress bounds (Figure 4.2). It proceeds till the overall strain reaches a value close to the recoverable strain predicted by the Taylor constraint strain bound. Intergranular constraints become significant at this point and we see martensite reorientation and hardening. Finally, even when the material appears to be fully saturated, there are significant regions that are untransformed. These insights are consistent with the observations of Brinson et al. [19].

- Interplay between plasticity and transformation. When the plastic yield strength is finite - higher than but of the same order of magnitude as the yield strength for transformation — plasticity sets in just as the intergranular constraints begin to impede the transformation. The material then begins to harden, and this results in plastic yielding in those isolated grains whose poor orientation impedes further transformation (Figure 4.3). An important insight of our work is that the material will harden to the extent necessary to provide enough plastic strain to overcome the incompatibility of transformation across misoriented grains. Consequently, the amount of the transformation and plastic strain as a function of the total imposed 
strain is universal in the sense that it is independent of the critical resolved stress of plasticity. This is shown in Figure 4.7. This partitioning seems to be robust even in the presence of moderate rate effects, as shown in Figure 4.12.

- Heterogeneity. The transformation and plasticity occur in an extremely heterogenous manner. The transformation begins in isolated grains and proceeds in bands of well-oriented grains. Plasticity provides a bridge across poorly oriented grains. Consequently the state of stress is extremely heterogeneous. Further, the nominal orientation and driving force are insufficient to describe the progress of transformation and plasticity (Figure 4.4).

- Residual strain and stress. The isolated regions of plasticity that appear on loading impede reverse transformation on unloading. Consequently, the stress at which reverse transformation occurs is lower and there is a residual strain and residual stress. An important observation of Figure 4.3 is that the residual strain is a result of a combination of both plastic yield and retained martensite (due to the residual stress field). This residual stress evolves with cycling but eventually settles down (Figure 4.8). The amount of residual strain is higher and it takes longer to settle down with lower critical resolved stress.

- Memory of deformation history. In uniaxial loading, the material that is unloaded and reloaded returns to a point that is close to the start of unloading and then continues along the virgin curve as shown in Figure 4.6. In multi-axial loading, the 
material quickly forgets its prior loading history when only a single mechanism is dominant (Figure 4.15(a),(d)), but retains longer memory when both mechanisms are active (Figure $4.15(\mathrm{~b}),(\mathrm{c}))$.

- Statistical homogeneity. In this work, we have shown results of a specimen with 512 grains. We see in Figure 4.9 that the results over various such specimens are indistinguishable. We also see from Figure 4.14 that the material response is extremely symmetric when subjected to non-proportional loads. Thus, we conclude that in this setting, 512 grains result in statistically isotropic and homogeneous materials. Indeed, we have observed that 128 grains is usually sufficient, but we see higher fluctuations.

The constitutive laws used in the anti-plane shear study can be thought of as the firstorder Taylor expansions of any arbitrarily complex constitutive laws which give physically realistic behavior. We emphasize that the simplicity of the chosen kinetic laws is not a limitation of the framework, but was utilized to clearly establish a major result of the antiplane shear study: that even in the absence of an explicit coupling between transformation and plasticity, a clear interplay is observed in the the full-field polycrystalline response.

In Chapter 5, we have generalized the framework to the 3-D setting, in which the convexified potential is a lower bound on the free energy, and we have included workhardening, and thermal- and rate-sensitivities. We have shown that the discrete time formulation of the generalized framework has a variational principle governing the update 
for mechanical equilibrium, and we have proposed an extension of the framework to finite kinematics, making use of the exponential mapping (approximating logarithmic strain).

We have demonstrated the suitability of the generalized framework to investigate polycrystalline materials other than SMAs. We have implemented the framework to model the $\alpha-\gamma($ BCC to FCC) transition in pure iron in a Taylor impact simulation. We have used this model to demonstrate the scalability of the framework in a large-scale, parallelized implementation on Caltech's Shared Heterogeneous Cluster, in which we see a nearly ideal (linear) time scale-up for up to 256 MPI processes.

Further, we have developed a model for $\alpha-\varepsilon$ (BCC to HCP) transition in iron, and have made an initial estimate for the material parameters by matching to a previous JohnsonCook model that had been fit to experimental shear data. In this implementation, we have made an preliminary attempt to account for deformation twining in the parent phase by extending the concept of the Bain strain, introducing a twinned $\alpha$-phase. We have implemented this model for a simulated the Taylor bar experiment, and a type of SplitHopkinson Pressure Bar experiment at various velocities.

Looking to the future, we again note that the the above SMA type behaviors were exhibited with no explicit coupling between the volume fraction and plastic slip parameters: all interaction between the two phenomena was communicated strictly through the elastic stress field, and influenced by the polycrystalline effect. With this baseline established, however, we envision the anti-plane shear model being used to explore more complex kinetic laws, including hardening and explicit coupling between plasticity and transformation, to 


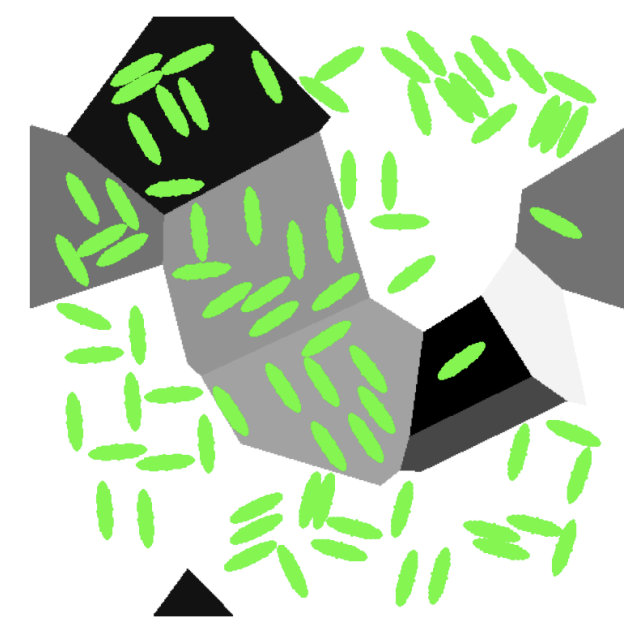

Figure 7.1: Simulated Voronoi microstruture with non-transforming inclusions (green) for anti-plane shear study. (Figure courtesy of D. Sun.)

gauge their relative effect on the macroscopic polycrystalline response.

Further, we note that the augmented Lagrangian solver implemented for the antiplane shear model is uniquely well-suited for handling materials with large contrasts in elastic properties. A study is currently underway to investigate the effects of soft and rigid non-transforming intragranular inclusions embedded in the polycrystal, varying the size, distribution, and crystallographic alignment of asymmetric inclusions; one realization of such a microstructure is shown in Figure 7.1.

Presently, efforts are underway to make direct comparison of the Split-Hopkinson Pressure Bar simulations to published data for iron. Extracting the simulated incident, reflected, and transmitted pulses from the numerical simulations should allow for such a comparison, and enable us to better determine the material parameters for the iron model.

Lastly, we have noted that the convexified potential, while exact for the setting of 
anti-plane shear, is only a lower bound for the actual energy potential that arises in the generalized 3-D setting. Other bounds exist, with the upper bounds given by rank-1 or laminate convexification seeming to be the most frequently explored. In the future, we might consider tightening our lower bound approximation by investigating the polyconvex envelope. Briefly, we wish to solve the problem,

$$
\min _{\mathbf{F} \in \mathcal{K}} I[\mathbf{F}], \quad I[\mathbf{F}]=\int_{\Omega_{0}} W(\mathbf{F}) \mathrm{d} \mathbf{X}
$$

We require that $I[\mathbf{F}]$ be finite and bounded from below, and know a minimizing sequence $\mathbf{F}_{\nu} \rightarrow \mathbf{F}_{m}$ exists if $I[\mathbf{F}]$ is weakly lower semi-continuous (w.l.s.c.); we know $I[\mathbf{F}]$ is w.l.s.c. if $W(\mathbf{F})$ is quasiconvex, i.e.

$$
\forall \nabla \phi \in \mathcal{K}: \int_{\Omega_{0}} W(\mathbf{F}) \mathrm{d} \mathbf{X} \leq \int_{\Omega_{0}} W(\mathbf{F}+\nabla \phi) \mathrm{d} \mathbf{X}
$$

This is obviously true if $W(\mathbf{F})$ is fully convex in $\mathbf{F}$, and we have taken the convex envelope $W^{* *}(\mathbf{F})$ as our approximation. However, it is also true if $W(\mathbf{F})$ is merely a convex function of all the minors of $\mathbf{F}$, that is

$$
W(\mathbf{F})=g(M(\mathbf{F}))
$$

where $g$ is a convex function of the vector $M(\mathbf{F})=(\mathbf{F}, \operatorname{cof}(\mathbf{F}), \operatorname{det}(\mathbf{F}))$. Computing the polyconvex envelope of the microscale potential has proven to be non-trivial, but we hope 
to make progress on this in the future. 


\section{Bibliography}

[1] R. Abeyaratne, C. Chu, and R.D. James. Kinetics of materials with wiggly energies: Theory and application to the evolution of twinning microstructures in a cu-al-ni shape memory alloy. Philosophical Magazine A, 73(2):457-497, 1996.

[2] R. Abeyaratne and J.K. Knowles. Kinetic Relations and the Propagation of Phase Boundaries in Solids. Archive for Rational Mechanics and Analysis, 114:119-154, 1991.

[3] R. Abeyaratne and J.K. Knowles. Evolution of Phase Transitions: A Continuum Theory. Number v. 10. 2006.

[4] R. Ahluwalia, T. Lookman, A. Saxena, and R.C. Albers. Landau theory for shape memory polycrystals. Acta Materialia, 52:209-218, 2004.

[5] A. Artemev, Y. Jin, and A.G. Khachaturyan. Three-dimensional phase field model and simulation of cubic üítetragonal martensitic transformation in polycrystals. Philosophical Magazine A, 82(6), 2002.

[6] M.F. Ashby and D.R.H. Jones. Engineering Materials 2: An Introduction to Microstructures, Processing and Design. International Series on Materials Science and 
Technology. Elsevier Science, 2005.

[7] F. Auricchio and L. Petrini. A three-dimensional model describing stress-temperature induced solid phase transformations: solution algorithm and boundary value problems. International Journal for Numerical Methods in Engineering, 61(1097-0207):John Wiley \& Sons, Ltd.-836, 2004.

[8] F. Auricchio, A. Reali, and U. Stefanelli. A three-dimensional model describing stressinduced solid phase transformation with permanent inelasticity. International Journal of Plasticity, 23(0749-6419), 2007.

[9] J.M. Ball and R.D. James. Fine phase mixtures as minimizers of energy. Archive for Rational Mechanics and Analysis, 100(0003-9527):Springer Berlin / Heidelberg-52, 1987.

[10] F. Benassi and M. Alves. Pulse shaping in the split hopkinson pressure bar test. In IV Congresso Nacional de Engenharia Mecanica, volume 22, pages 1256-1263, 2006.

[11] K. Bhattacharya. Microstructure of Martensite: Why It Forms and How It Gives Rise to the Shape-Memory Effect. 2003.

[12] K. Bhattacharya, S. Conti, G. Zanzotto, and J. Zimmer. Crystal symmetry and the reversibility of martensitic transformations. Nature, 428:55-59, 2004.

[13] K. Bhattacharya, S. Conti, G. Zanzotto, and J. Zimmer. Crystal symmetry and the reversibility of martensitic transformations. Nature, 428(6978):55-59, 2004. 
[14] K. Bhattacharya and R.V. Kohn. Symmetry, texture and the recoverable strain of shape-memory polycrystals. Acta Materialia, 44:529-542, 1996.

[15] K. Bhattacharya and RV Kohn. Elastic energy minimization and the recoverable strains of polycrystalline shape-memory materials. Archive for Rational Mechanics and Analysis, 139:99-180, 1997.

[16] K. Bhattacharya and A. Schlöemerkemper. Stress-Induced Phase Transformations in Shape-Memory Polycrystals. Archive for Rational Mechanics and Analysis, 196:715751,2010

[17] R. Brenner, R.A. Lebensohn, and O. Castelnau. Elastic anisotropy and yield surface estimates of polycrystals. Int. J. Solids. Struct., 46:3018-3026, 2009.

[18] L.C. Brinson. One-dimensional constitutive behavior of shape memory alloys: Thermomechanical derivation with non-constant material functions and redefined martensite internal variable. Journal of Intelligent Material Systems and Structures, 4(2):229 $242,1993$.

[19] L.C. Brinson, I. Schmidt, and R. Lammering. Stress-induced transformation behavior of a polycrystalline NiTi shape memory alloy: micro and macromechanical investigations via in situ optimcal microscopy. Journal of the Mechanics and Physics of Solids, $52: 1549-1571,2004$. 
[20] F. Cardarelli. Materials Handbook: A Concise Desktop Reference. Springer London, Limited, 2008.

[21] D.S. Chandrasekharaiah and L. Debnath. Continuum mechanics. Academic Press, 1994.

[22] S. Chatterjee, M. Murugananth, and H.K.D.H. Bhadeshia. $\delta$ TRIP steel. Materials Science and Technology, 23(7):819-827, 2007-07-01.

[23] R.D. Coleman and W. Noll. The Thermodynamics of Elastic Materials with Heat Conduction and Viscosity. Archive for Rational Mechanics and Analysis, 13:167-178, 1963.

[24] A.M. Cuitino and M. Ortiz. A material-independent method for extending stress update algorithms from small-strain plasticity to finite plasticity with multiplicative kinematics. Engineering Computations, 9(4):437 - 451, 1992.

[25] A.M. Cuitino and M. Ortiz. Computational modelling of single crystals. Modelling and Simulation in Materials Science and Engineering, 1(3):225, 1993.

[26] S. Daly, G. Ravichandran, and K. Bhattacharya. Stress-induced martensitic phase transformation in thin sheets of Nitinol. Acta Materialia, 55:3593-3600, 2007.

[27] J.L. Ericksen. Introduction to the Thermodynamics of Solids, volume 131. Springer, 1998. 
[28] P. Franciosi. Glide mechanisms in b.c.c. crystals: An investigation of the case of -iron through multislip and latent hardening tests. Acta Metallurgica, 31(9):1331 - 1342, 1983.

[29] X. Gao, M. Huang, and L.C. Brinson. A multivariant micromechanical model for SMAs Part 1. Crystallographic issues for single crystal model. Int. J. Plasticity, $16: 1345-1369,2000$.

[30] P.M. Giles, M.H. Longenbach, and A.R. Marder. High-pressure $\alpha \leftrightarrow \varepsilon$ martensitic transformation in iron. J. Appl. Phys., 42:4290-4295, 1971.

[31] S. Govindjee, A. Mielke, and G.J. Hall. The free energy of mixing for n-variant martensitic phase transformations using quasi-convex analysis. Journal of the Mechanics and Physics of Solids, 51(0022-5096), 2003.

[32] F. Grennerat, M. Montagnat, O. Castelnau, P. Vacher, H. Moulinec, P. Suquet, and P. Duval. Experimental characterization of the intragranular strain field in columnar ice during transient creep. Acta Mater., 60:3655-3666, 2012.

[33] M.E. Gurtin. An Introduction to Continuum Mechanics. Mathematics in Science and Engineering. Elsevier Science, 1982.

[34] R. Heinen and K. Hackl. On the calculation of energy-minimizing phase fractions in shape memory alloys. Computer Methods in Applied Mechanics and Engineering, 196(0045-7825), 2007. 
[35] J.P. Hirth and J. Lothe. Theory of Dislocations, 2nd. 1982.

[36] M.I. Idiart, H. Moulinec, P. Ponte Castañeda, and P. Suquet. Macroscopic behavior and field fluctuations in viscoplastic composites: Second-order estimates versus fullfield simulations. Journal of the Mechanics and Physics of Solids, 54:1029-1063, 2006.

[37] A. Kelly. A constitutive relation for shape-memory alloys. PhD thesis, California Institute of Technology, 2009.

[38] M. Kimiecik, J.W. Jones, and S. Daly. Quantitative studies of microstructural phase transformation in nickel-titanium. Materials Letters, 95(0):25-29, 2013.

[39] H. Kolsky. An investigation of the mechanical properties of materials at very high rates of loading. Proceedings of the Physical Society. Section B, 62(11):676, 1949.

[40] D.C. Lagoudas, P.B. Entchev, P. Popov, E. Patoor, L.C. Brinson, and X. Gao. Shape memory alloys, part II: Modeling of polycrystals. Mechanics of Materials, 38(01676636), 2006.

[41] R.A. Lebensohn. N-site modeling of a 3D viscoplastic polycrystal using Fast Fourier Transform. Acta Materialia, 49(14):2723 - 2737, 2001.

[42] R.A. Lebensohn, R. Brenner, O. Castelnau, and A.D. Rollett. Orientation image-based micromechanical modelling of subgrain texture evolution in polycrystalline copper. Acta Mater., 54:3914-3926, 2008. 
[43] R.A. Lebensohn, C.N. Tome, and P. Ponte Castañeda. Self-consistent modeling of the mechanical behavior of viscoplastic polycrystals incorporating intragranular field fluctuations. Phil. Mag, 87:4287-4322, 2007.

[44] J.B. Leblond. Mathematical modelling of transformation plasticity in steels ii: Coupling with strain hardening phenomena. International Journal of Plasticity, 5(6):573$591,1989$.

[45] J.B. Leblond, G. Mottet, and J.C. Devaux. A theoretical and numerical approach to the plastic behaviour of steels during phase transformations - ii. study of classical plasticity for ideal-plastic phases. Journal of the Mechanics and Physics of Solids, 34(0022-5096), 1986.

[46] S. Leclercq and C. Lexcellent. A general macroscopic description of the thermomechanical behavior of shape memory alloys. Journal of the Mechanics and Physics of Solids, 44(0022-5096), 1996.

[47] E.H. Lee. Some comments on elastic-plastic analysis. International Journal of Solids and Structures, 17(9):859-872, 1981.

[48] V.I. Levitas, A.V. Idesman, and D.L. Preston. Microscale simulation of martensitic microstructure evolution. Phys. Rev. Lett., 93(10), Sep 2004. 
[49] V.I. Levitas and D.L. Preston. Three-dimensional landau theory for multivariant stress-induced martensitic phase transformations. i. austenite $\leftrightarrow$ martensite. Phys. Rev. B, 66:134206, Oct 2002 .

[50] A. Lew, K. Caspersen, E.A. Carter, and M. Ortiz. Quantum mechanics based multiscale modeling of stress-induced phase transformations in iron. Journal of the Mechanics and Physics of Solids, 54(6):1276-1303, 2006.

[51] C. Lexcellent and A. Schlömerkemper. Comparison of several models for the determination of the phase transformation yield surface in shape-memory alloys with experimental data. Acta Materialia, 55(1359-6454), 2007.

[52] B. Li. The optimal transportation method in solid mechanics. PhD thesis, California Institute of Technology, 2009.

[53] B. Li, F. Habbal, and M. Ortiz. Optimal transportation meshfree approximation schemes for fluid and plastic flows. International journal for numerical methods in engineering, 83(12):1541-1579, 2010.

[54] J. Lubliner. Plasticity theory. Dover Publications, 2008.

[55] B. Malard, P. Sittner, M. Berveiller, and E. Patoor. Advances in martensitic transformations in Cu-based shape memory alloys achieved by in situ neutron and synchrotron X-ray diffraction methods. C.R. Physique, 13:13-13, 2012. 
[56] H.-K. Mao, W.A. Bassett, and T. Takahashi. Effect of pressure on crystal structure and lattice parameters of iron up to 300 kbar. Journal of Applied Physics, 38(1):272$276,1967$.

[57] F. Marketz and F.D. Fischer. Micromechanical modelling of stress-assisted martensitic transformation. Mod. Sim. Mat. Sci. Engng., pages 1017-1046, 1994.

[58] J.M. McNaney, V. Imbeni, Y. Jung, P. Papadopoulos, and R.O. Ritchie. An experimental study of the superelastic effect in a shape-memory Nitinol alloy under biaxial loading. Mech. Mater., 35:969-986, 2003.

[59] J.C. Michel, H. Moulinec, and P. Suquet. A computational scheme for linear and non-linear composites with arbitrary phase contrast. Int. J. Numer. Meth. Engng., $52: 139-160,2001$.

[60] C. Morin, Z. Moumni, and W. Zaki. Thermomechanical coupling in shape memory alloys under cyclic loadings: Experimental analysis and constitutive modeling. Int. J. Plasticity, 27:1959-1980, 2011.

[61] S. Nemat-Nasser. On finite deformation elasto-plasticity. International Journal of Solids and Structures, 18(10):857-872, 1982.

[62] S. Nemat-Nasser. Plasticity: a treatise on finite deformation of heterogeneous inelastic materials. Cambridge University Press, 2004. 
[63] S. Nemat-Nasser, T. Okinaka, and L. Ni. A physically-based constitutive model for bcc crystals with application to polycrystalline tantalum. Journal of the Mechanics and Physics of Solids, 46(6):1009 - 1038, 1998.

[64] V. Novák and P. Šittner. Micromechanics modelling of niti polycrystalline aggregates transforming under tension and compression stress. Materials Science and Engineering: A, 378(1):490-498, 2004.

[65] M. Ortiz, R.A. Radovitzky, and E.A. Repetto. The computation of the exponential and logarithmic mappings and their first and second linearizations. International Journal for Numerical Methods in Engineering, 52(12):1431-1441, 2001.

[66] M. Ortiz and L. Stainier. The variational formulation of viscoplastic constitutive updates. Computer methods in applied mechanics and engineering, 171(3):419-444, 1999.

[67] K. Otsuka and C.M. Wayman. Shape-memory Materials. Cambridge University Press, 1999.

[68] M. Panico and L.C. Brinson. A three-dimensional phenomenological model for martensite reorientation in shape memory alloys. Journal of the Mechanics and Physics of Solids, 55(0022-5096), 2007. 
[69] E. Patoor, D.C. Lagoudas, P.B. Entchev, L.C. Brinson, and X. Gao. Shape memory alloys, part I: General properties and modeling of single crystals. Mechanics of Materials, 38(0167-6636), 2006.

[70] R. Phillips. Crystals, defects and microstructures: modeling across scales. Cambridge University Press, 2001.

[71] M.A. Qidwai and D.C. Lagoudas. On thermomechanics and transformation surfaces of polycrystalline niti shape memory alloy material. International Journal of Plasticity, 16(10-11):1309-1343, 2000.

[72] J.R. Rice. Inelastic constitutive relations for solids: An internal-variable theory and its application to metal plasticity. Journal of the Mechanics and Physics of Solids, 19(0022-5096), 1971.

[73] J.R. Rice. Inelastic constitutive relations for solids: An internal-variable theory and its application to metal plasticity. Journal of the Mechanics and Physics of Solids, 19:433-455, 1971.

[74] A.W. Richards, R.A. Lebensohn, and K. Bhattacharya. Interplay of martensitic phase transformation and plastic slip in polycrystals. Acta Materialia, 61(12):4384-4397, 2013

[75] A.W. Richards, B. Li, K. Bhattacharya, and M. Ortiz. A three-dimensional model of the interplay between transformation and plasticity. 2013. 
[76] D. Rittel, G. Ravichandran, and A. Venkert. The mechanical response of pure iron at high strain rates under dominant shear. Materials Science and Engineering: A, 432(1):191-201, 2006.

[77] R.T. Rockafellar. Convex analysis. Princeton Landmarks in Mathematics and Physics Series. Princeton University Press, 1997.

[78] F. Roters, P. Eisenlohr, L. Hantcherli, D.D. Tjahjanto, T.R. Bieler, and D. Raabe. Overview of constitutive laws, kinematics, homogenization and multiscale methods in crystal plasticity finite-element modeling: Theory, experiments, applications. Acta Materialia, 58(1359-6454), 2010.

[79] F. Roters, P. Eisenlohr, L. Hantcherli, D.D. Tjahjanto, T.R. Bieler, and D. Raabe. Overview of constitutive laws, kinematics, homogenization and multiscale methods in crystal plasticity finite-element modeling: Theory, experiments, applications. Acta Materialia, 59:1152-1211, 2010.

[80] A. Sadjadpour and K. Bhattacharya. A micromechanics inspired constitutive model for shape-memory alloys: the one-dimensional case. Smart materials and structures, 16(1):S51, 2007.

[81] A. Sadjapour, D. Rittel, G. Ravichandran, and K. Bhatacharya. Dynamic deformation of iron under shear. Unpublished experimental data and Johnson-Cook model parameters, 2008. 
[82] Y.C. Shu and K. Bhattacharya. The influence of texture on the shape-memory effect in polycrystals. Acta Materialia, 46:5457-5473, 1998.

[83] J.C. Simo and T.J.R. Hughes. Computational Inelasticity. 1998.

[84] P. Sittner, Y. Hara, and M. Tokuda. Experimental study on the thermoelastic martensitic transformation in shape memory alloy polycrystal induced by combined external forces. Metallurgical and Materials Transactions A, 26:2923-2935, 1995. 10.1007/BF02669649.

[85] V.P. Smyshlyaev and J.R. Willis. A 'non-local' variational approach to the elastic energy minimalization of martensitic polycrystals. $P$ R Soc A, 454:1573-1613, 1998.

[86] A.C. Souza, E.N. Mamiya, and N. Zouain. Three-dimensional model for solids undergoing stress-induced phase transformations. European Journal of Mechanics A/Solids, 17(0997-7538), 1998.

[87] A.J.M. Spencer. Continuum Mechanics. Dover Publications, Incorporated, 2004.

[88] A. Stebner, X. Gao, D.W. Brown, and L.C. Brinson. Neutron diffraction studies and multivariant simulations of shape memory alloys: Empirical texture developmentmechanical response relations of martensitic nickel-titanium. Acta Materialia, $59: 2841-2849,2011$.

[89] P.M. Suquet and K. Bhattacharya. A model problem concerning recoverable strains of shape-memory polycrystals. Proc. Roy. Soc. Lond. A, 461:2797-2816, 2005. 
[90] L. Taleb and F. Sidoroff. A micromechanical modeling of the greenwood-johnson mechanism in transformation induced plasticity. International Journal of Plasticity, 19(0749-6419), 2003.

[91] G. Taylor. The use of flat-ended projectiles for determining dynamic yield stress. i. theoretical considerations. Proceedings of the Royal Society of London. Series A. Mathematical and Physical Sciences, 194(1038):289-299, 1948.

[92] D. Tjahjanto, S. Turteltaub, and A. Suiker. Crystallographically based model for transformation-induced plasticity in multiphase carbon steels. Continuum Mechanics and Thermodynamics, 19(0935-1175):399-422, 2008.

[93] Y.D. Wang, X.-L. Wang, A.D. Stoica, J.W. Richardson, and R. Lin Peng. Determination of the stress orientation distribution function using pulsed neutron sources. Journal of Applied Crystallography, 36(1):14-22, Feb 2003.

[94] M.L. Wilkins and M.W. Guinan. Impact of cylinders on a rigid boundary. Journal of Applied Physics, 44(3):1200-1206, 1973.

[95] K. Yamauchi, I. Ohkata, K. Tsuchiya, and S. Miyazaki, editors. Shape Memory and Superelastic Alloys: Technologies and Applications. Woodhead Publishers, 2011.

[96] H.L. Yi, K.Y. Lee, and H.K.D.H. Bhadeshia. Extraordinary ductility in al-bearing $\delta$-TRIP steel. Proceedings of the Royal Society A: Mathematical, Physical and Engineering Science, 467(2125):234-243, 2011. 